Article

\title{
About Calculation of Massless and Massive Feynman Integrals
}

\author{
Anatoly V. Kotikov(1) \\ Bogoliubov Laboratory of Theoretical Physics, Joint Institute for Nuclear Research, 141980 Dubna, Russia; \\ kotikov@theor.jinr.ru
}

Received: 15 April 2020; Accepted: 28 April 2020; Published: 7 May 2020

check for updates

\begin{abstract}
We report some results of calculations of massless and massive Feynman integrals particularly focusing on difference equations for coefficients of for their series expansions.
\end{abstract}

Keywords: Feynman integrals; dimensional regularization; Gegenbauer Polynomials

\section{Introduction}

Calculation of Feynman integrals gives basic information about both experimentally investigated processes and the characteristics of physical models. The calculations of the matrix elements of the processes under study depend on the masses of the particles involved in the interaction and, strictly speaking, require the calculation of Feynman integrals including those with massive propagators. Depending on the kinematics of the studied processes, the values of some masses can be neglected.

The study of the characteristics of physical models (for example, critical parameters, anomalous dimensions of particles and operators) usually requires calculations of massless Feynman integrals, which have a much simpler structure. They allow one to obtain results for these characteristics in high orders of perturbation theory.

Note that when calculating Feynman integrals, it is advisable to use analytical methods whenever possible. The fact is that approximate methods for calculating Feynman integrals do not always have sufficiently high accuracy. Moreover, the calculation of Feynman integrals is ambiguous for numerical calculations both due to the singular nature of the integrals themselves and (especially for gauge theories) with strong mutual contractions between the contributions of different diagrams or even between parts of the same diagram.

Moreover, in many important situations it is necessary to know exactly the exact results. For example, in the framework of renormalization group calculations in theories with high internal symmetry, it is important to know [1] about the vanishing of $\beta$-functions in the order under consideration.

We note that when using dimensional regularization [2-5], i.e., for an arbitrary dimension of space, once found diagrams for any field model (or process) can be applied to other models (and processes), since the main ones are scalar diagrams. Consequently, the complexity of analytical calculations of Feynman integrals pays off for their universality in application to various quantum-field models.

We also note the fact that the calculation of complicated diagrams may have some independent interest. So the use of non-trivial identities such as the "uniqueness" relation [6,7] can give information (see [8-13]) about the properties of some integrals and series that are not yet in the reference literature. For example, calculations of the same Feynman integral carried out in Refs. [8] and [14] using different calculation methods made it possible to find a previously unknown relation 
between hypergeometric functions with arguments 1 and -1 . This relation has been proven only just recently [15].

Recently, many powerful original methods have appeared for calculating Feynman integrals (see, a recent review in [16]), which are often inferior in width to standard ones, i.e., the $\alpha$-presentation and technique of Feynman parameters (see, for example, [1,17]), however, can significantly advance in the number of loops for a limited range of quantities (or processes).

In this paper, we consider massless diagrams that contribute to the coefficient functions and anomalous dimensions for the process of deep inelastic scattering (DIS) of leptons by hadrons. Then we will show the main steps in calculating diagrams with massive propagators. We also show that the moments of structure functions (SFs) and expansion coefficients (in inverse mass) have many common features. The same or similar complex sums arise there and there, which can be conveniently calculated using recursive relationships between them.

Such similarity is not strange. First of all, the moments of the DIS structure functions are not calculated directly. The connection between deep inelastic scattering and elastic forward scattering is used, which is realized through the optical theorem. The forward elastic scattering amplitudes are determined by (scalar) diagrams depending on two momenta, $q$ and $p$ with the condition $p^{2}=0$. Such diagrams can be expanded in series with respect to $(p q) / Q^{2}$ and, in particular, the structure of the coefficients of these series is similar to the corresponding structure of the expansion coefficients for the inverse mass in massive diagrams (here we consider the case of only one mass). This similarity of the structures of the coefficients is not surprising, a similar similarity of the results of the calculation of these types of diagrams was discussed long ago in Ref. [18].

In Sections 2-4 we demonstrate the development of the multiloop calculation methods, as applied to diagrams containing an arbitrary number of derivatives (or momenta) on lines. Such diagrams arise when using the "gluing" method [19] and the method "projectors" [20-23] for calculating the SF moments of deep inelastic scattering of leptons at hadrons in QCD.

Contrary to original papers [24-26] all calculations are carried out in $p$-space. We note that original results have been done in $x$-space for so-called dual diagrams (see, for example, $[14,24,26]$ ). A dual diagram is obtained from the initial one by replacement of all $p$ by $x$ with the rules of correspondence between the graph and the integral, as in a $x$-space. The transition to the dual diagram is indicated by $\stackrel{d}{=}$.

Of course, the results of the integration of the diagrams do not changed during the procedure. However the graphic representations are different. Shortly speaking, all loops (triangles, $n$-leg one-loop internal graphs) should be replaced by the corresponding chains (three-leg vertices, $n$-leg vertices). With the usage of the dual technique, the evaluation of the $\alpha_{s}$-corrections to the longitudinal DIS structure function has been done in [24,27]. All the calculations were done for the massless diagrams. The extension of such calculations to the massive case were done in [28,29]. Some recent evaluations of the massive dual Feynman integrals can be found also in [30].

In Sections 3 and 4 we present several examples of calculations of massless diagrams. An introductions to the basic steps of the methods of "gluing" and "projectors" is given in Appendix B. The calculation of massive diagrams is given in Sections 5 and 6. Additional rules are given for their efficient calculation, examples of two- and three-point diagrams are considered. The recurrence relations for the coefficients of decomposition in inverse mass are considered. A short review of modern computing technology is given.

To calculate the most complex parts of massless and massive diagrams, recurrence relations for the coefficients of their decomposition are used (currently, there are also popular recursive relationships (see [31-35]) for diagrams with different values of space, however, their consideration is above slope of the present paper). Solving these recursive relationships allows you to get accurate results for these most complex parts. Obtaining these results is discussed in detail in Sections 3 and 6 for massless and massive diagrams, respectively. 
A popular property of maximal transcendentality is shown in Section 7. It was introduced in [36] for the Balitsky-Fadin-Kuraev-Lipatov (BFKL) kernel [37-44] in the $\mathcal{N}=4$ Supersymmetric Yang-Mills (SYM) model [45,46], is also applicable for the anomalous dimension matrices of the twist-2 and twist-3 Wilson operators and for the coefficient functions of the "deep-inelastic scattering" in this model. The property gives a possibility to recover the results for the anomalous dimensions [47-50] and the coefficient functions [51] without any direct calculations by using the QCD corresponding values [52-54].

The very similar property appears also in the results of calculation of the large class of Feynman integrals, mostly for so-called master integrals [55]. The results for most of them can be reconstructed also without any direct calculations using a knowledge of several terms in their inverse-mass expansion [56,57]. Note that the properties of the results are related with the ones of the amplitudes, form-factors and correlation functions (see [58-107] and references therein) studied recently in the framework of the $\mathcal{N}=4$ SYM.

In Section 6, we demonstrate the existence of the propertiy of maximal transcendentality (or maximal complexity) in the results of two-loop two- and three-point Feynman integrals (see also [108-112]).

\section{Basic Formulas}

Let us briefly consider the rules for calculation of massless diagrams. All calculations are carried out in momentum space.

Following to Ref. [113], we introduce the traceless product $q^{\mu_{1} \ldots \mu_{n}}$ of the momentums connected with the usual product $q^{\mu_{1}} \ldots q^{\mu_{n}}$ by the following equations

$$
\begin{aligned}
q^{\mu_{1} \ldots \mu_{n}} & =\hat{S} \sum_{p \geq 0} \frac{(-1)^{p} n ! \Gamma(n-p+\lambda)}{2^{2 p}(n-2 p) ! p ! \Gamma(n+\lambda)} g^{\mu_{1} \mu_{1}} \ldots g^{\mu_{2 p-1} \mu_{2 p}} q^{2 p} q^{\mu_{2 p+1}} \ldots q^{\mu_{n}}, \\
q^{\mu_{1}} \ldots q^{\mu_{n}} & =\hat{S} \sum_{p \geq 0} \frac{n ! \Gamma(n-2 p+1+\lambda)}{2^{2 p}(n-2 p) ! p ! \Gamma(n-p+1+\lambda)} g^{\mu_{1} \mu_{1} \ldots g^{\mu_{2 p-1} \mu_{2 p}} q^{2 p} q^{\mu_{2 p+1} \ldots \mu_{n}},}
\end{aligned}
$$

where $\hat{S}$ is a symmetrization on indeces $\mu_{i}(i=1, \ldots, n)$.

We give also the simple but quite useful conditions:

$$
\left(q_{1} q_{2}\right)^{(n)} \equiv q_{1}^{\mu_{1} \ldots \mu_{n}} q_{2}^{\mu_{1} \ldots \mu_{n}}=q_{1}^{\mu_{1}} \ldots q_{1}^{\mu_{n}} q_{2}^{\mu_{1} \ldots \mu_{n}}=q_{1}^{\mu_{1} \ldots \mu_{n}} q_{2}^{\mu_{1}} \ldots q_{2}^{\mu_{n}}
$$

which follow immediately from the traceless-product definition: $g^{\mu_{i} \mu_{j}} q^{\mu_{1} \ldots \mu_{i} \ldots \mu_{j} \ldots \mu_{n}}=0$.

Propagators will be represented as

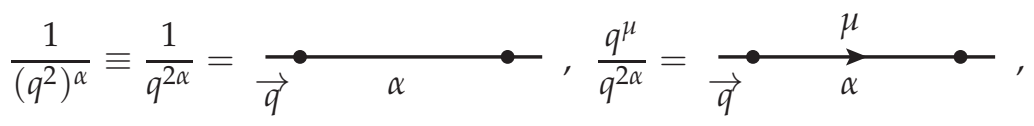

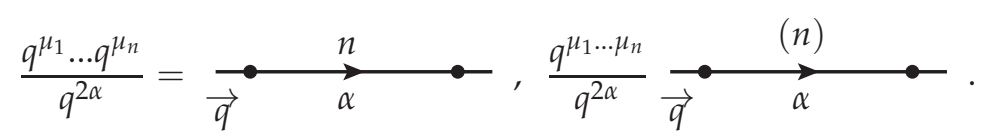

The use of the traceless product $q^{\mu_{1} \ldots \mu_{n}}$ makes it possible to ignore terms of the type $g^{\mu_{i} \mu_{j}}$ that arise upon integration: they can be readily recovered from the general structure of the traceless product. Therefore, in the process of integration it is only necessary to follow the coefficient of the leading term $q^{\mu_{1}} \ldots q^{\mu_{n}}$.

Everywhere in the paper on arguments $k, k_{1}, k_{2} \ldots$ integration is carried out in $d=4-2 \varepsilon$-space. Hereafter in Sections $3-5$ the labels $k, k_{1}, k_{2} \ldots$ denote integnal momentums. The symbols $q_{1} q_{1}, q_{2} \ldots$ and $p, p_{1}, p_{2} \ldots$ denote the external mometums with the conditions $p^{2}=0, p_{1}^{2}=0, p_{2}^{2}=0 \ldots$, respectively. 
The following formulas hold (a traceless product can also be used to calculate complicated diagrams in another way. A propagator of complicated diagram can be expanded in a series of two other propagators, having traceless products in their numerators. This technique [14,113] is very effective for evaluating complicated scalar diagrams with propagators having arbitrary degrees (see Refs. [114-121] and the review [122]).

A. For simple chain:

$$
\frac{q^{\mu_{1}} \ldots q^{\mu_{n}}}{q^{2 \alpha_{1}}} \frac{q^{v_{1}} \ldots q^{v_{m}}}{q^{2 \alpha_{2}}}=\frac{q^{\mu_{1}} \ldots q^{\mu_{n}} q^{\nu_{1}} \ldots q^{v_{m}}}{q^{2\left(\alpha_{1}+\alpha_{2}\right)}}
$$

or graphically

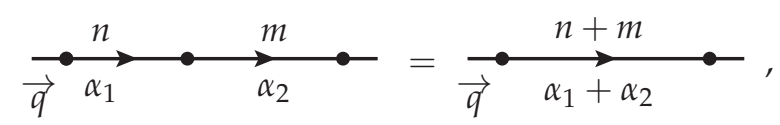

i.e., the product of propagators is equivalent to a new propagator with an index equal to the sum of the indices of the original propagators. The number of momentums in the product in the numerator is equal to the sum of the products of the impulses in the original propagators.

B. A simple loop can be integrated as

$$
\int \frac{D k k^{\mu_{1}} \ldots k^{\mu_{n}}}{(q-k)^{2 \alpha_{1}} k^{2 \alpha_{2}}}=\frac{1}{(4 \pi)^{d / 2}} \frac{q^{\mu_{1}} \ldots q^{\mu_{n}}}{q^{2\left(\alpha_{1}+\alpha_{2}-d / 2\right)}} A^{0, n}\left(\alpha_{1}, \alpha_{2}\right)+\ldots,
$$

where we neglect the terms of the order $g^{\mu_{i} \mu_{j}}$. Here

$$
D k=\frac{d^{d} k}{(2 \pi)^{d}} .
$$

is usual integration in Euclidean measure and

$$
A^{n, m}(\alpha, \beta)=\frac{a_{n}(\alpha) a_{m}(\beta)}{a_{n+m}(\alpha+\beta-d / 2)}, \quad a_{n}(\alpha)=\frac{\Gamma(\tilde{\alpha}+n)}{\Gamma(\alpha)}, \quad \tilde{\alpha}=\frac{d}{2}-\alpha .
$$

It is convenient to rewrite the equation graphically as

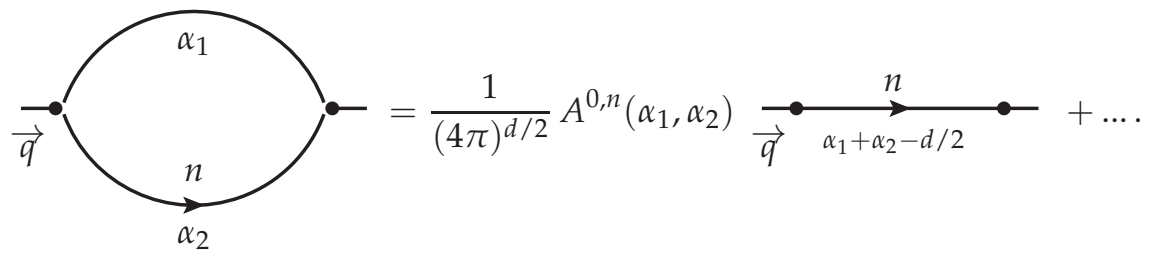

For the corresponding traceless product Equation (7) becomes to be exact, i.e.,

$$
\int \frac{D k k^{\mu_{1} \ldots \mu_{n}}}{(q-k)^{2 \alpha_{1}} k^{2 \alpha_{2}}}=\frac{1}{(4 \pi)^{d / 2}} \frac{q^{\mu_{1} \ldots \mu_{n}}}{q^{2\left(\alpha_{1}+\alpha_{2}-d / 2\right)}} A^{0, n}\left(\alpha_{1}, \alpha_{2}\right),
$$

or graphically

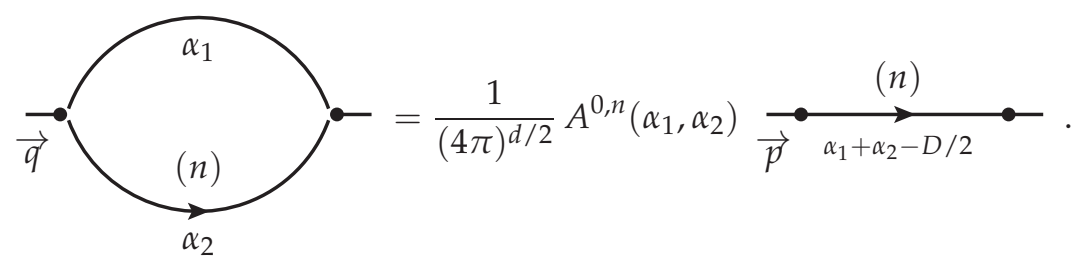


As it was noted already, indeed, we use the transless product $q^{\mu_{1} \ldots \mu_{n}}$ in the r.h.s. but really we need only the first term, i.e., $q^{\mu_{1}} \ldots q^{\mu_{n}}$, because the rest (i.e., the terms containing $g^{\mu_{i} \mu_{j}}$ ) is exactly reconstracted from the exact form of traceless production.

Such property can be used in another way: a convenient representation of the results (7) and (8) can be obtained also by using an extra lightlike momentum $u$ (i.e., with $u^{2}=0$ ) and considering the product $(u k)^{n}=u_{\mu_{1}} \ldots u_{\mu_{n}} k^{\mu_{1}} \ldots k^{\mu_{n}}=u_{\mu_{1}} \ldots u_{\mu_{n}} k^{\mu_{1} \ldots \mu_{n}}$, because $u^{2}=0$.

The results have the following form

$$
\int \frac{D k(u k)^{n}}{(q-k)^{2 \alpha_{1}} k^{2 \alpha_{2}}}=\frac{1}{(4 \pi)^{d / 2}} \frac{(u q)^{n}}{q^{2\left(\alpha_{1}+\alpha_{2}-d / 2\right)}} A^{0, n}\left(\alpha_{1}, \alpha_{2}\right),
$$

or graphically

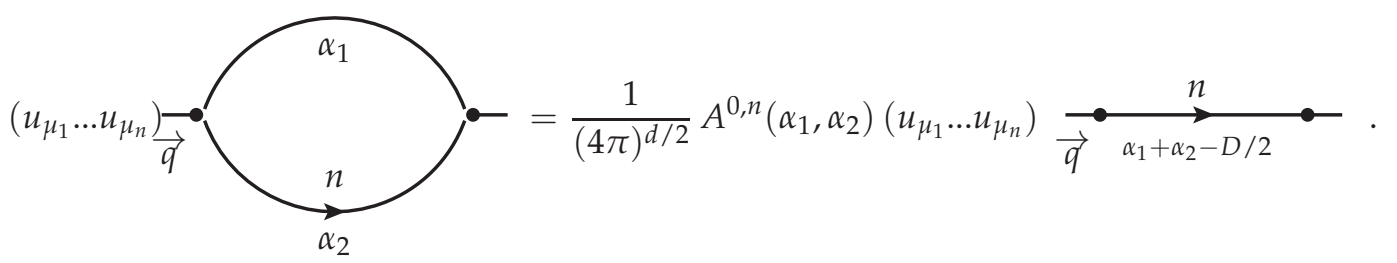

Note that we use everything $\mu_{i}$ belonging to the traceless product, i.e., we consider the case of a scalar diagrams with a traceless product. In real theories such as QCD, there are still other indices $\lambda_{j}$, which correspond to the numerators of propagators. In such cases, we can no longer neglect the terms $g^{\lambda_{i} \lambda_{j}}$ and $g^{\mu_{i}} \lambda_{j}$ and, therefore, the integration rules are complicated. They can be found in Refs. [24-26].

So, all diagrams, which can be expressed as combinations of loops and chains can be evaluated immediately. However, starting already with the two-loop level, there are diagrams, which cannot be expressed as combinations of loops and chains (simplest example is shown below in Figure 1). For these cases there are additional rules, which will be shown only graphically with a purpose to increase an illustation power.

C. When $\sum \alpha_{i}=d$, there is so-called uniqueness ratio [6-12] for the triangle with indices $\alpha_{i}$ $(i=1,2,3)$

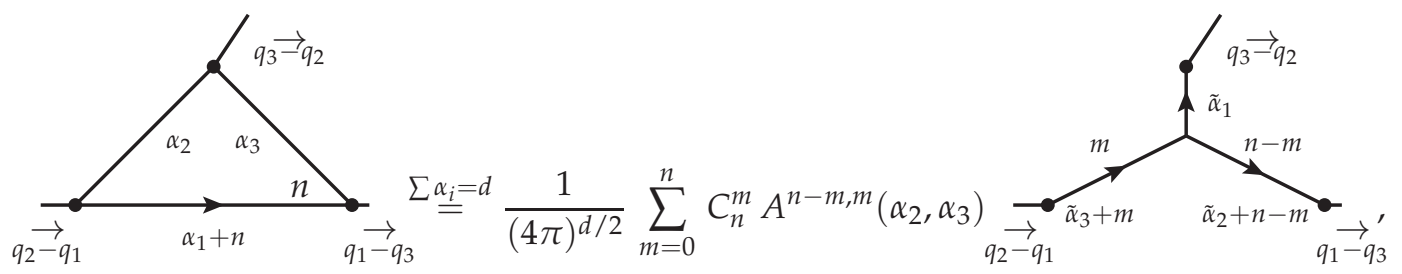

where

$$
C_{n}^{m}=\frac{n !}{m !(n-m) !} .
$$

The results (10) can be exactlly obtained in the following way: perform an inversion $q_{i} \rightarrow 1 / q_{i}$ $(i=1,2,3), k \rightarrow 1 / k$ in the subintegral expression and in the integral measure. The inversion keeps angles between momentums. After the inversion, one propagator is cancelled because $\sum \alpha_{i}=d$ and the 1.h.s. becomes to be equal to a loop. Evaluating it using the rule (7) and returning after it to the initial momentums, we recover the rule (10). An extension of the rule (10) to the case with two traceless products can be found in [26].

D. For any triangle with indices $\alpha_{i}(i=1,2,3)$ there is the following relation, which is based on integration by parts (IBP) procedure $[7,123,124]$ 


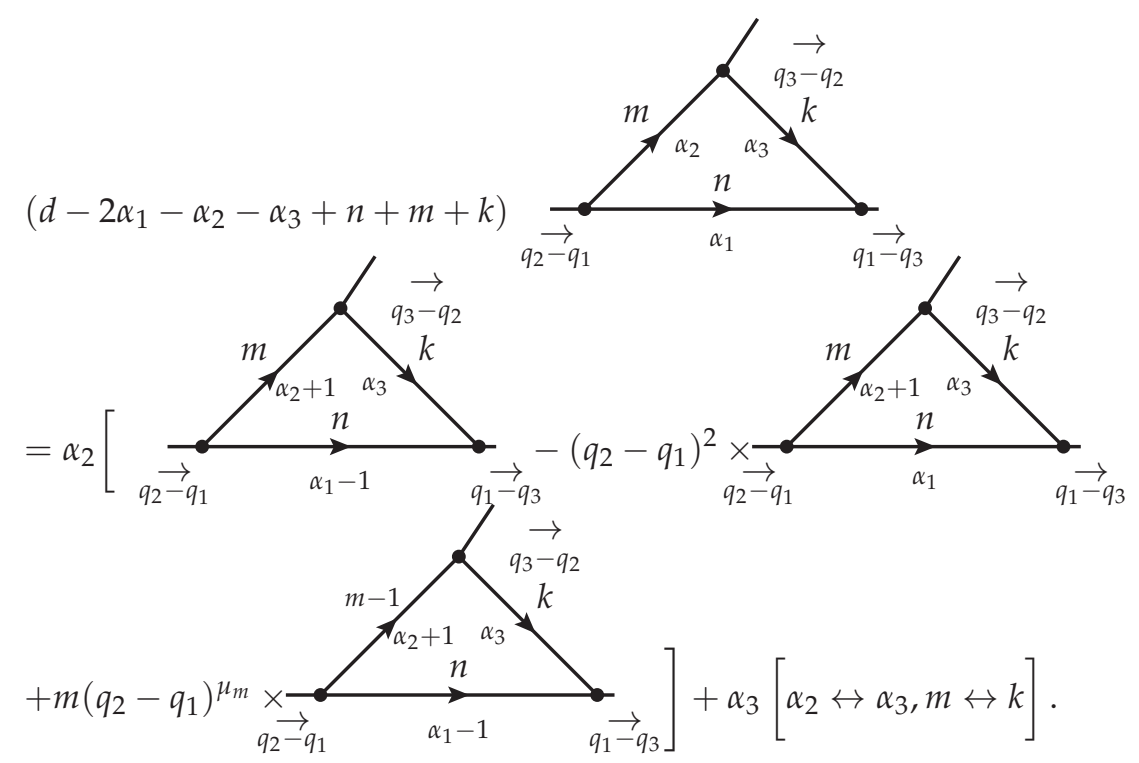

Equation (12) can been obtained by introducing the factor $\left(\partial / \partial k_{\mu}\right)\left(k-q_{1}\right)^{\mu}$ to the subintegral expression of the triangle, shown below as [...], and using the integration by parts procedure as follows:

$$
\begin{aligned}
d \int D k[\ldots]= & \int D k\left(\frac{\partial}{\partial k_{\mu}}\left(k-q_{1}\right)^{\mu}\right)[\ldots]=\int D k \frac{\partial}{\partial k_{\mu}}\left(\left(k-q_{1}\right)^{\mu}[\ldots]\right) \\
& -\int D k\left(k-q_{1}\right)^{\mu} \cdot \frac{\partial}{\partial k_{\mu}}([\ldots])
\end{aligned}
$$

The first term in the r.h.s. becomes to be zero because it can be represented as a surphase integral on the infinite surphase. Evalutiong the second term in the r.h.s. we reproduce Equation (12).

As it is possible to see from Equations (12) and (13) the line with the index $\alpha_{1}$ is distingulished. The contributions of the other lines are same. So, we will call below the line with the index $\alpha_{1}$ as a "distingulished line". It is clear that a various choices of the distingulished line produce different tipes of the IBP relations.

Using Equation (12) allows you to change the indices of the line diagrams by an integer. One can also change line indices using the point group of transformations $[7,125,126]$. The elements of the group are: (a) the transition to impulse presentation, (b) conformal inversion transformation $p \rightarrow$ $p^{\prime}=p / p^{2}$, (c) a special series of transformations that makes it possible to make one of the vertices unique, and then apply relation (3) to it. An extension of the group of transformations for diagrams with the traceless product can be found in Ref. [26].

\section{Basic Massless Two-Loop Integrals}

The general topology of the two-loop two-point diagram, which cannot be expressed as a combination of loops and chanins is shown on Figure 1.

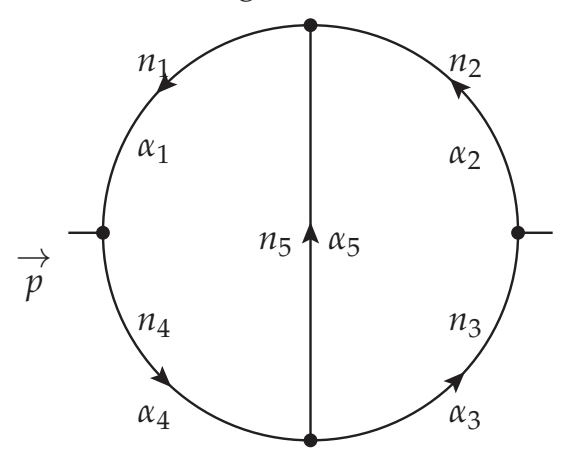

Figure 1. The diagram which cannot be expressed as a combination of loops and chains. 
Below in the present analysis we will concentrate mostly on two particular cases, which can be taken from the diagram shown in Figure 1 , for $\alpha_{3}+n_{3}=\alpha, n_{3}=n, \alpha_{j}(j \neq 3)=1, n_{j}(j \neq 3)=0$ (we denote $\left.I_{1}(\alpha, n)\right)$ and for $\alpha_{5}+n_{5}=\alpha, n_{5}=n, \alpha_{j}(j \neq 5)=1, n_{j}(j \neq 5)=0\left(\right.$ we denote $\left.I_{2}(\alpha, n)\right)$

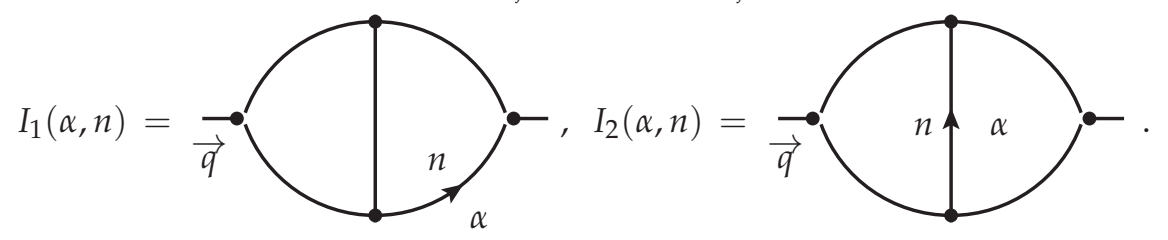

It is convenient to calculate the diagrams $I_{1}(\alpha, n)$ and $I_{2}(\alpha, n)$ using the functional relations (the functional relations were obtained in $[13,127]$ by application of IBP realtions with various distinguished lines) similar to those obtained in $[13,127]$, which reduces the amount of computations.

Repeating analysis done in [13], we obtain the following functional relations:

$$
\begin{aligned}
& I_{1}(\alpha, n)=\frac{1}{q^{2}} I_{1}(\alpha-1, n)-\frac{1}{2 \varepsilon}\left(2 I_{1,1}(\alpha, n)+I_{2,1}(\alpha, n)\right), \\
& I_{2}(\alpha, n)=\frac{2}{n+d-2-2 \alpha} I_{2,1}(\alpha, n)-\frac{n+2 d-4-2 \alpha}{n+d-2-2 \alpha} \frac{1}{q^{2}} I_{2}(\alpha-1, n),
\end{aligned}
$$

where the inhomogeneus terms are

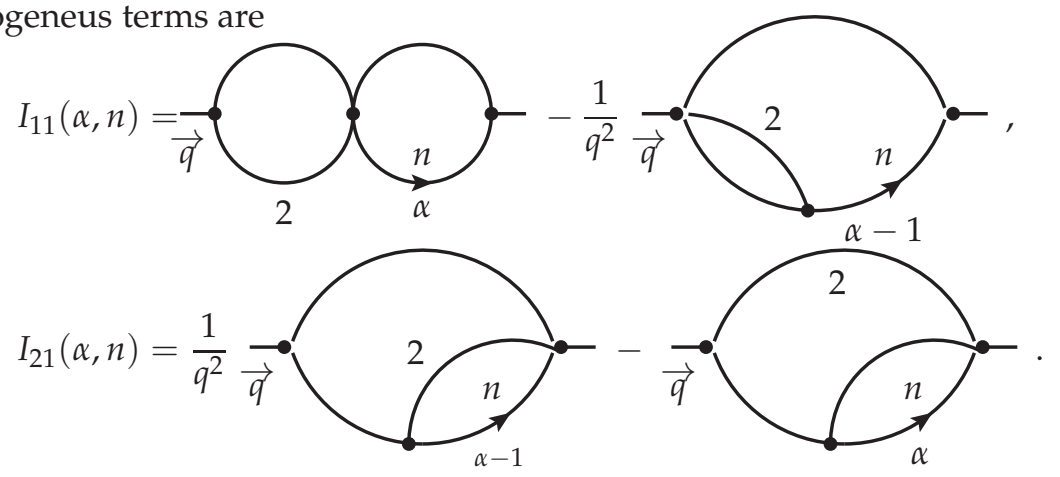

We see that the inhomogeneus terms in the functional Equations (15) and (16), i.e. the diagrams $I_{11}(\alpha, n)$ and $I_{21}(\alpha, n)$, can be represented as combinations of loops and chains and, thus, they can be evaluated by rules (4) and (7).

We would like to note that for the massless two-point diagrams the subject of the study is so-called coefficient functions, which expression $C_{i \ldots}(\alpha, n)(i=1,2)$ for the considered diagrams $I_{i \ldots}(\alpha, n)$ can be introduced in the following form

$$
I_{i \ldots}(\alpha, n)=\frac{1}{(4 \pi)^{d}} C_{i \ldots}(\alpha, n) \frac{q^{\mu_{1} \ldots \mu_{n}}}{q^{2(\alpha+2 \varepsilon)}} .
$$

The result (19) contains the fact that we consider the two-loop diagrams. In general, L-loop diagram $I_{L}\left(\alpha_{1}, \ldots, \alpha_{N}, n\right)$ having propagators with indices $\alpha_{i}(i=1, \ldots, N)$ and one traceless product of momentums can be respresented as

$$
I_{L}\left(\alpha_{1}, \ldots, \alpha_{N}, n\right)=\frac{1}{(4 \pi)^{d L / 2}} C_{L}\left(\alpha_{1}, \ldots, \alpha_{N}, n\right) \frac{q^{\mu_{1} \ldots \mu_{n}}}{q^{2(\bar{\alpha}-d / 2 * \varepsilon)}},
$$

where $\bar{\alpha}=\sum_{1}^{N} \alpha_{i}$. 
The results for $C_{i \ldots}(\alpha, n)$ can be obtained directly from rules (4) and (7). They have the following form:

$$
\begin{aligned}
& C_{1,1}(\alpha, n)=A(2,1)\left(A^{0, n}(1, \alpha)-A^{0, n}(1, \alpha+\varepsilon)\right), \\
& C_{2,1}(\alpha, n)=A^{0, n}(2, \alpha-1) A^{0, n}(1, \alpha+\varepsilon)-A^{0, n}(1, \alpha) A^{0, n}(2, \alpha+\varepsilon),
\end{aligned}
$$

where

$$
A\left(\alpha_{1}, \alpha_{2}\right)=A^{0,0}\left(\alpha_{1}, \alpha_{2}\right) .
$$

and the result for $A^{n, m}\left(\alpha_{1}, \alpha_{2}\right)$ is given in (6).

Thus, the coefficient functions $C_{i, 1}(\alpha, n)(i=1,2)$ can be represented as combinations of $\Gamma$-functions.

\section{1. $I_{1}(0, n)$ and $I_{2}(0, n)$}

The diagrams $I_{1}(0, n)$ and $I_{2}(0, n)$ can be considered a s natural boundary conditions for the functional Equations (15) and (16). Moreover, in a sence, they have a special property: their results can be obtained with help of Equations (4) and (7) but with an additional resummatiomn.

Indeed, expanding for $I_{1}(0, n)$ and $I_{2}(0, n)$, respectively, the corresponding products of momentums in the following way:

$$
\begin{array}{ll}
\prod_{i=1}^{n}\left(q-k_{2}\right)^{\mu_{i}}=\sum_{k=0}^{n} C_{n}^{k}(-1)^{k} \prod_{i=1}^{k} k_{2}^{\mu_{i}} \prod_{j=1}^{n-k} q^{\mu_{j}} & \text { for } I_{1}(0, n), \\
\prod_{i=1}^{n}\left(k_{1}-k_{2}\right)^{\mu_{i}}=\sum_{k=0}^{n} C_{n}^{k}(-1)^{k} \prod_{i=1}^{k} k_{2}^{\mu_{i}} \prod_{j=1}^{n-k} k_{1}^{\mu_{j}} & \text { for } I_{2}(0, n),
\end{array}
$$

we see that the diagrams $I_{1}(0, n)$ and $I_{2}(0, n)$ can be represented as

$$
\begin{aligned}
& I_{1}(0, n)=\sum_{k=0}^{n} C_{n}^{k}(-1)^{k} \prod_{j=1}^{n-k} q^{\mu_{j}} \bar{I}_{1}(0, k), \\
& I_{2}(0, n)=\sum_{k=0}^{n} C_{n}^{k}(-1)^{k} \bar{I}_{2}(n-k, k),
\end{aligned}
$$

where:

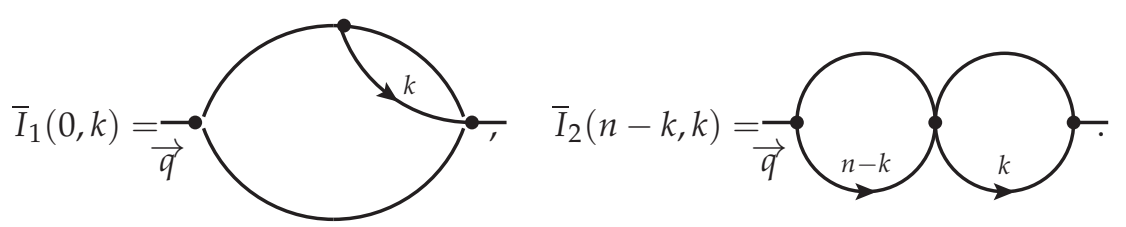

So, the diagrams $I_{1}(0, n)$ and $I_{2}(0, n)$ can be expressed as combination of loops and chains and, thus, their coefficient functions can be calculated using rules (4) and (7).

So, we have for the coefficient functions $C_{1}(0, n)$ and $C_{2}(0, n)$ :

$$
\begin{gathered}
C_{1}(0, n)=\sum_{k=0}^{n} C_{n}^{k}(-1)^{k} A^{0, k}(1,1) A^{0, k}(1,1+\varepsilon), \\
C_{2}(0, n)=\sum_{k=0}^{n} C_{n}^{k}(-1)^{k} A^{0, k}(1,1) A^{0, n-k}(1,1) .
\end{gathered}
$$

As we noted already at the beginning of the subsection, the results for $C_{i}(0, n)(i=1,2)$ are very important because they give a possibility to recover all results $C_{i}(m, n)$ using Equations (15) and (16) 
with $\alpha=m$. But the calculation of $C_{i}(0, n)$ needs performing additional series (see Equations (29) and (30)). So, we present these calculations in some details, separetely for $I_{1}(0, n)$ and $I_{2}(0, n)$ in the following two subsections.

3.2. $I_{1}(0, n)$

Now we consider $C_{1}(0, n)$ form Equation (29), which can be represented as

$$
C_{1}(0, n)=\frac{N_{2}}{2 \varepsilon^{2}} \sum_{k=0}^{n} C_{n}^{k}(-1)^{k} \frac{\Gamma(k+1-\varepsilon) \Gamma(1-3 \varepsilon)}{\Gamma(k+2-3 \varepsilon) \Gamma(1-\varepsilon)} \frac{1}{k+1-2 \varepsilon} .
$$

It is conveninet to rewrite the last term in the r.h.s. as

$$
\frac{1}{k+1-2 \varepsilon}=\frac{1}{k+1}+\frac{2 \varepsilon}{(k+1)(k+1-2 \varepsilon)}
$$

that leads to the following form for $C_{1}(0, n)$ :

$$
C_{1}(0, n)=\bar{C}_{1}(0, n)+2 \varepsilon \tilde{C}_{1}(0, n)
$$

with

$$
\begin{aligned}
& \bar{C}_{1}(0, n)=\frac{N_{2}}{2 \varepsilon^{2}} \sum_{k=0}^{n} C_{n}^{k}(-1)^{k} \frac{\Gamma(k+1-\varepsilon) \Gamma(1-3 \varepsilon)}{\Gamma(k+2-3 \varepsilon) \Gamma(1-\varepsilon)} \frac{1}{k+1}, \\
& \tilde{C}_{1}(0, n)=\frac{N_{2}}{2 \varepsilon^{2}} \sum_{k=0}^{n} C_{n}^{k}(-1)^{k} \frac{\Gamma(k+1-\varepsilon) \Gamma(1-3 \varepsilon)}{\Gamma(k+2-3 \varepsilon) \Gamma(1-\varepsilon)} \frac{1}{(k+1)(k+1-2 \varepsilon)},
\end{aligned}
$$

where the normalization $N_{2}$ and the factors $K_{1}$ and $K_{2}$ are

$$
N_{2}=\Gamma^{2}(1+\varepsilon) K_{1} K_{2}, \quad K_{1}=\frac{\Gamma^{2}(1-\varepsilon)}{\Gamma(1-2 \varepsilon)}, \quad K_{1}=\frac{\Gamma(1-\varepsilon) \Gamma(1-2 \varepsilon) \Gamma(1+2 \varepsilon)}{\Gamma^{2}(1+\varepsilon) \Gamma(1-3 \varepsilon)} .
$$

The result for $\bar{C}_{1}(0, n)$ can be found exactly as

$$
\begin{aligned}
& \frac{\bar{C}_{1}(0, n)}{N_{2} /\left(2 \varepsilon^{2}\right)}=\sum_{k=1}^{n+1} \frac{(-1)^{k+1} n !}{k !((n-k+1) !)} \frac{\Gamma(k-\varepsilon) \Gamma(1-3 \varepsilon)}{\Gamma(k+1-3 \varepsilon) \Gamma(1-\varepsilon)} \\
& =-\frac{1}{n+1}\left[{ }_{2} F_{1}(-(n+1),-\varepsilon ; 1-3 \varepsilon ; 1)-1\right] \frac{\Gamma(-e p)}{\Gamma(1-e p)} \\
& =\frac{1}{(n+1) \varepsilon}[B(n+2,-2,-3)-1]=\frac{1}{(n+1) \varepsilon}\left[\frac{n+1-2 \varepsilon}{n+1-3 \varepsilon} B(n+1,-2,-3)-1\right],
\end{aligned}
$$

where

$$
B(n+1, a, b)=\frac{\Gamma(n+1+a \varepsilon) \Gamma(1+b \varepsilon)}{\Gamma(1+a \varepsilon) \Gamma(n+1+b \varepsilon)} .
$$

The result for $B(n+1, a, b)$ can be easily evaluated using the expansion of the corresponding $\Gamma$-functions [56]

$$
\begin{aligned}
& \frac{\Gamma(n+1+a \varepsilon)}{n ! \Gamma(1+a \varepsilon)}=\exp \left[-\sum_{m=1}^{\infty} \frac{(-a \varepsilon)^{m}}{m} S_{m}(n)\right], \\
& \Gamma(1+a \varepsilon)=\exp \left[-\gamma a \varepsilon+\sum_{m=1}^{\infty} \frac{(-a \varepsilon)^{m}}{m} \zeta_{m}\right],
\end{aligned}
$$

where $\gamma$ is Euler's constant. 
Indeed, we have

$$
\begin{aligned}
& B(n+1, a, b)=\exp \left[-\sum_{m=1}^{\infty} \frac{(-\varepsilon)^{m}}{m}\left[a^{m}-b^{m}\right] S_{m}(n)\right]=1+(a-b) \varepsilon S_{1}(n) \\
& +\frac{(a-b) \varepsilon^{2}}{2}\left[(a-b) S_{1}^{2}(n)-(a+b) S_{2}(n)\right]+\frac{(a-b) \varepsilon^{3}}{3 !} \\
& \times\left[(a-b)^{2} S_{1}^{3}(n)-3\left(a^{2}-b^{2}\right) S_{1}(n) S_{2}(n)+2\left(a^{2}+a b+b^{2}\right) S_{3}(n)\right]+O\left(\varepsilon^{4}\right) .
\end{aligned}
$$

Hereafter we use the following nested sums:

$$
S_{ \pm i}(n)=\sum_{m=1}^{n} \frac{(-1)^{m}}{m^{i}}, S_{ \pm i, j}(n)=\sum_{m=1}^{n} \frac{(-1)^{m}}{m^{i}} S_{ \pm j}(m),
$$

and $\zeta(n)=S_{n}(\infty)$-Riemann zeta-function.

The result for $\tilde{C}_{1}(0, n)$ can be expressed as

$$
\frac{\tilde{C}_{1}(0, n)}{N_{2} /\left(2 \varepsilon^{2}\right)}=\sum_{k=0}^{n} C_{n}^{k}(-1)^{k} \frac{1}{(k+1)^{3}}\left(1+\varepsilon\left[2 S_{1}(k)+\frac{5}{k+1}\right]\right)+O\left(\varepsilon^{2}\right) .
$$

To evaluate the r.h.s it is convenient to calculate firstly the series $\phi_{1}(a, n)$ and $\Phi_{1}(a, n)$

$$
\phi_{1}(a, n)=\sum_{k=0}^{n} C_{n}^{k}(-1)^{k} \frac{1}{k+1-a}, \quad \Phi_{1}(a, n)=\sum_{k=0}^{n} C_{n}^{k}(-1)^{k} \frac{S_{1}(k)}{k+1-a},
$$

differentiate them several times with respect of $a$ and put $a=0$. Indeed,

$$
\frac{\tilde{C}_{1}(0, n)}{N_{2} /\left(2 \varepsilon^{2}\right)}=\phi_{1}^{(2)}(n)+\varepsilon\left(2 \Phi_{1}^{(2)}(n)+5 \phi_{1}^{(3)}(n)\right)+O\left(\varepsilon^{2}\right),
$$

where

$$
\phi_{1}^{(m)}(n)=\left.\frac{1}{m !} \frac{\partial^{m}}{(\partial a)^{m}} \phi_{1}(a, n)\right|_{a=0}, \Phi_{1}^{(m)}(n)=\left.\frac{1}{m !} \frac{\partial^{m}}{(\partial a)^{m}} \Phi_{1}(a, n)\right|_{a=0}
$$

The series $\phi_{1}(a, n)$ can be calculated directly as

$$
\phi_{1}(a, n)=\frac{1}{1-a}{ }_{2} F_{1}(-n, 1-a, 2-a)=\frac{\Gamma(1-a) \Gamma(n+1)}{\Gamma(n+2-a)}
$$

and we have

$$
\begin{aligned}
& \phi_{1}(n, 0)=\frac{1}{n+1}, \phi_{1}^{(1)}(n)=\frac{1}{n+1} S_{1}(n+1) \\
& \phi_{1}^{(2)}(n)=\frac{1}{2(n+1)}\left(S_{1}^{2}(n+1)+S_{2}(n+1)\right) \\
& \phi_{1}^{(3)}(n)=\frac{1}{6(n+1)}\left(S_{1}^{3}(n+1)+3 S_{1}(n+1) S_{2}(n+1)+2 S_{3}(n+1)\right) .
\end{aligned}
$$

Evaluating the series $\Phi_{1}(a, n)$ is quite difficut, and we will show this in a separate subsection. The calculation of more complicated series can be found in a famous paper [128]. 


\subsection{1. $\Phi_{1}(a, n)$}

It is convenient to consider the recursive relation between $\Phi_{1}(a, n)$ and $\Phi_{1}(a, n-1)$. Indeed, we can represented $\Phi_{1}(a, n)$ in the following form

$$
\Phi_{1}(a, n)=\sum_{k=0}^{n-1} \frac{(-1)^{k} n !}{k !(n-1-k) !} \frac{S_{1}(k)}{(n-k)(k+1-a)}+(-1)^{n} \frac{S_{1}(n)}{n+1-a} .
$$

Taking partial fraction

$$
\frac{1}{(n-k)(k+1-a)}=\frac{1}{n+1-a}\left[\frac{1}{n-k}+\frac{1}{k+1-a}\right],
$$

we can rewrite (47) in the following way

$$
\Phi_{1}(a, n)=\frac{1}{n+1-a}\left[n \Phi_{1}(a, n)+\sum_{k=0}^{n} \frac{(-1)^{k} n !}{k !(n-k) !} S_{1}(k)\right] .
$$

The series in the r.h.s. can be calculated usng results for $\hat{\Phi}_{1}(\alpha, \beta)$, studied in Appendix $A$, as

$$
\begin{aligned}
& \sum_{k=0}^{n} \frac{(-1)^{k} n !}{k !(n-k) !} S_{1}(k)=\left.\frac{\partial}{(\partial x)} \sum_{k=0}^{n} \frac{(-1)^{k} n ! \Gamma(1-x)}{\Gamma(k+1-x)(n-k) !}\right|_{x=0} \\
& =\left.n ! \frac{\partial}{(\partial x)}\left\{\Gamma(1-x) \hat{\Phi}_{1}(\alpha=0, \beta=1-x)\right\}\right|_{x=0}=\left.\frac{\partial}{(\partial x)} \frac{x}{x-n}\right|_{x=0}=-\frac{1}{n} .
\end{aligned}
$$

It is convenient to introduce the new function $\bar{\Phi}_{1}(a, n)$ as

$$
\Phi_{1}(a, n)=\frac{\Gamma(1-a) \Gamma(n+1)}{\Gamma(n+2-a)} \Phi_{1}(a, n),
$$

which has the following relation:

$$
\bar{\Phi}_{1}(a, n)=\bar{\Phi}_{1}(a, n-1)-\frac{\Gamma(n+1-a)}{n ! \Gamma(1-a)} \frac{1}{n} .
$$

The last relation can be solved as

$$
\bar{\Phi}_{1}(a, n)=\bar{\Phi}_{1}(a, 0)-\sum_{m=1}^{n} \frac{\Gamma(m+1-a)}{m ! \Gamma(1-a)} \frac{1}{m} .
$$

Since $\Phi_{1}(a, n=0)=0$, then $\bar{\Phi}_{1}(a, 0)=0$ and we find finally

$$
\Phi_{1}(a, n)=-\frac{\Gamma(1-a) \Gamma(n+1)}{\Gamma(n+2-a)} \sum_{m=1}^{n} \frac{\Gamma(m+1-a)}{m ! \Gamma(1-a)} \frac{1}{m}
$$

and for $a=0$

$$
\Phi_{1}(0, n)=-\frac{1}{n+1} \sum_{m=1}^{n} \frac{1}{m}=-\frac{S_{1}(n)}{n+1} .
$$

Note that the case $\Phi_{1}(0, n)$ can be evaluated directly in the way similar to the one (37) for $\bar{C}_{1}(0, n)$ and there is a full agreement of such calculations with (54). 
After little algebra, we have

$$
\begin{aligned}
\Phi_{1}^{(1)}(n)= & -\frac{1}{n+1} \sum_{m=1}^{n} \frac{1}{m}\left(S_{1}(n+1)-S_{1}(m)\right) \\
& =-\frac{1}{n+1}\left[\frac{1}{2}\left(S_{1}^{2}(n)-S_{2}(n)\right)+\frac{S_{1}(n)}{n+1}\right], \\
\Phi_{1}^{(2)}(n)= & -\frac{1}{2(n+1)} \sum_{m=1}^{n} \frac{1}{m}\left[\left(S_{1}(n+1)-S_{1}(m)\right)^{2}+S_{2}(n+1)-S_{2}(m)\right] \\
& =-\frac{1}{2(n+1)}\left[\frac{1}{3}\left(S_{1}^{3}(n)-3 S_{1}(n) S_{2}(n)-4 S_{3}(n)\right)+2 S_{2,1}(n)\right. \\
& \left.+\frac{1}{n+1}\left(S_{1}^{2}(n)-S_{2}(n)\right)+\frac{2 S_{1}(n)}{(n+1)^{2}}\right]
\end{aligned}
$$

where we used the formulas from the part C of Appendix A.

We would like to note about an appearence the nested sum $S_{2,1}(n)$, which cannot be obtained from expansions of products of $\Gamma$-functions.

3.2.2. $C_{1}(0, n)$

Taking the results for $\phi_{1}^{(2)}(n), \phi_{1}^{(3)}(n)$ and $\Phi_{1}^{(2)}(n)$ together, we can obtain the following results for $\tilde{C}_{1}(0, n)$

$$
\frac{\tilde{C}_{1}(0, n)}{N_{2} /\left(2 \varepsilon^{2}\right)}=\frac{\varepsilon}{n+1-2 \varepsilon}\left[\frac{1}{2} C(n)+\varepsilon D(n)\right]+O\left(\varepsilon^{3}\right),
$$

where

$$
\begin{aligned}
& \hat{C}(n)=S_{1}^{2}(n)+S_{2}(n)+\frac{2 S_{1}(n)}{n+1}+\frac{2}{(n+1)^{2}}=S_{1}^{2}(n+1)+S_{2}(n+1), \\
& \hat{D}(n)=\bar{D}(n)+\frac{1}{2(n+1)}\left(S_{1}^{2}(n)+5 S_{2}(n)\right)+\frac{S_{1}(n)}{(n+1)^{2}}+\frac{3}{(n+1)^{3}}
\end{aligned}
$$

and

$$
\bar{D}(n)=\frac{1}{2}\left(S_{1}^{3}(n)+7 S_{1}(n) S_{2}(n)+6 S_{3}(n)-4 S_{2,1}(n)\right)
$$

We note that there is another representation for $\tilde{C}_{1}(0, n)$ :

$$
\frac{\tilde{C}_{1}(0, n)}{N_{2} /\left(2 \varepsilon^{2}\right)}=\frac{\varepsilon}{n+1}\left[\frac{1}{2} C(n)+\varepsilon \bar{D}(n+1)\right]+O\left(\varepsilon^{3}\right) \text {. }
$$

Taking the results for $\bar{C}_{1}(0, n)$ and $\tilde{C}_{1}(0, n)$ of (61) together, we have the final result for $C_{1}(0, n)$ :

$$
\begin{aligned}
& C_{1}(0, n)=\frac{N_{2}}{2(n+1)}\left[\frac{1}{\varepsilon^{2}} S_{1}(n+1)+\frac{1}{2 \varepsilon}\left(3 S_{1}^{2}(n+1)+7 S_{2}(n+1)\right)\right. \\
& \left.+\frac{7}{6} S_{1}^{3}(n+1)+\frac{19}{2} S_{1}(n+1) S_{2}(n+1)+\frac{37}{3} S_{3}(n+1)-4 S_{2,1}(n+1)\right]
\end{aligned}
$$

with the normalization factor $N_{2}$ was defined in (36).

Since $K_{2} / K_{1} \sim O\left(\varepsilon^{3}\right)$ (see Equation (114) below), we can replace in the r.h.s. the normalization $N_{2}$ by $N_{1}$

$$
N_{1}=\Gamma^{2}(1+\varepsilon) K_{1}^{2}
$$

and put the normalization $N_{1}$ in the definition of $\mu_{g}^{2}$-scale of $g$-scheme [129], which relates with the usual $\overline{M S}$ one as $\mu_{g}^{2}=K_{1} \mu_{\overline{M S}}^{2}$ (see discussions in Ref. [130,131]). 
3.3. $I_{2}(0, n)$

The diagram $I_{2}(0, n)$ s zero for odd $n$ values. So, we can calculate it firstly at the even $n$ values and recover its genetal form at the end.

So, now we consider $C_{2}(0, n)$ from Equation (30), which can be represented as

$$
C_{2}(0, n)=\frac{N_{1}}{\varepsilon^{2}} \sum_{k=0}^{n} C_{n}^{k}(-1)^{k} \frac{\Gamma(k+1-\varepsilon) \Gamma(n-k+1-\varepsilon) \Gamma^{2}(1-2 \varepsilon)}{\Gamma(k+2-3 \varepsilon) \Gamma(n-k+2-2 \varepsilon) \Gamma^{2}(1-\varepsilon)},
$$

where the normalization factor $N_{1}$ is given in Equation (63).

The last part of r.h.s. can be written as (for even $n$ )

$$
\begin{aligned}
& \frac{B(k+1,-1,-2) B(n-k+1,-1,-2)}{(k+1-2 \varepsilon)(n-k+1-2 \varepsilon)}=\frac{2}{n+2-4 \varepsilon} \frac{1}{k+1-2 \varepsilon} \\
& \times B(k+1,-1,-2) B(n-k+1,-1,-2)
\end{aligned}
$$

because

$$
\frac{1}{(k+1-2 \varepsilon)(n-k+1-2 \varepsilon)}=\frac{2}{n+2-4 \varepsilon}\left[\frac{1}{k+1-2 \varepsilon}+\frac{1}{n-k+1-2 \varepsilon}\right]
$$

Moreover, with the required accuracy $O\left(\varepsilon^{0}\right)$, we can rewrite the product $B(k+1,-1,-2) B(n-$ $k+1,-1,-2)$ in the following way:

$$
\begin{aligned}
& B(k+1,-1,-2) B(n-k+1,-1,-2)=B(k+1,-1,-2) \\
& +B(n-k+1,-1,-2)-1+\varepsilon^{2} S_{1}(k) S_{1}(n-k)+O\left(\varepsilon^{2}\right)
\end{aligned}
$$

because

$$
B(k+1,-1,-2)=1+\varepsilon S_{1}(n)+\frac{\varepsilon^{2}}{2}\left(S_{1}^{2}(n)-3 S_{2}(n)\right)+O\left(\varepsilon^{2}\right) .
$$

Then, $C_{2}(0, n)$ can be splitted to the four different parts:

$$
C_{2}(0, n)=\frac{2}{n+2-4 \varepsilon}\left[C_{2}^{(1)}(0, n)+C_{2}^{(2)}(0, n)-C_{2}^{(3)}(0, n)+\varepsilon^{2} C_{2}^{(4)}(0, n)\right]
$$

where

$$
\begin{aligned}
& C_{2}^{(1)}(0, n)=\frac{N_{1}}{\varepsilon^{2}} \sum_{k=0}^{n}(-1)^{k} C_{N}^{k} \frac{B(k+1,-1,-2)}{k+1-2 \varepsilon}, \\
& C_{2}^{(2)}(0, n)=\frac{N_{1}}{\varepsilon^{2}} \sum_{k=0}^{n}(-1)^{k} C_{N}^{k} \frac{B(n-k+1,-1,-2)}{k+1-2 \varepsilon} \\
& C_{2}^{(3)}(0, n)=\frac{N_{1}}{\varepsilon^{2}} \sum_{k=0}^{n}(-1)^{k} C_{N}^{k} \frac{1}{k+1-2 \varepsilon} \\
& C_{2}^{(4)}(0, n)=\sum_{k=0}^{n}(-1)^{k} C_{N}^{k} \frac{S_{1}(k) S_{1}(n-k)}{k+1}
\end{aligned}
$$


The parts $C_{2}^{(1)}(0, n)$ and $C_{2}^{(3)}(0, n)$ can be evaluated directly. Indeed,

$$
\begin{aligned}
& \frac{C_{2}^{(1)}(0, n)}{N_{1} / \varepsilon^{2}}=\sum_{k=0}^{n}(-1)^{k} C_{N}^{k} \frac{\Gamma(k+1-\varepsilon) \Gamma(1-2 \varepsilon)}{\Gamma(k+2-2 \varepsilon) \Gamma(1-\varepsilon)}=\frac{1}{1-2 \varepsilon} 2 F_{1}(-n, 1-\varepsilon ; 2-2 \varepsilon ; 1) \\
& =\frac{\Gamma(n+1-\varepsilon) \Gamma(1-2 \varepsilon)}{\Gamma(n+2-2 \varepsilon) \Gamma(1-\varepsilon)}=\frac{B(n+1,-1,-2)}{(n+1-2 \varepsilon)}, \\
& \frac{C_{2}^{(3)}(0, n)}{N_{1} / \varepsilon^{2}}=\frac{1}{1-2 \varepsilon} 2 F_{1}(-n, 1-2 \varepsilon ; 2-2 \varepsilon ; 1)=\frac{\Gamma(n+1) \Gamma(1-2 \varepsilon)}{\Gamma(n+2-2 \varepsilon)}=\frac{B(n+1,0,-2)}{(n+1-2 \varepsilon)} .
\end{aligned}
$$

As in the case of $C_{1}(0, n)$, using Equation (32) it is convenient to split the part $C_{2}^{(2)}(0, n)$ in two parts

$$
C_{2}^{(2)}(0, n)=\bar{C}_{2}^{(2)}(0, n)+2 \varepsilon \tilde{C}_{2}^{(2)}(0, n),
$$

where

$$
\begin{aligned}
& \bar{C}_{2}^{(2)}(0, n)=\frac{N_{1}}{\varepsilon^{2}} \sum_{k=0}^{n} C_{n}^{k}(-1)^{k} \frac{B(n-k+1,-1,-2)}{k+1}, \\
& \tilde{C}_{2}^{(2)}(0, n)=\frac{N_{1}}{\varepsilon^{2}} \sum_{k=0}^{n} C_{n}^{k}(-1)^{k} \frac{B(n-k+1,-1,-2)}{(k+1)(k+1-2 \varepsilon)} .
\end{aligned}
$$

As in the case of $\bar{C}_{1}(0, n)$, the part $\bar{C}_{2}^{(2)}(0, n)$ can be found exactly:

$$
\begin{aligned}
& \frac{\bar{C}_{2}^{(2)}(0, n)}{N_{2} / \varepsilon^{2}}=\sum_{k=1}^{n+1} \frac{(-1)^{k+1} n !}{k !((n-k+1) !)} \frac{\Gamma(n-k+2-\varepsilon) \Gamma(1-2 \varepsilon)}{\Gamma(n-k+3-2 \varepsilon) \Gamma(1-\varepsilon)} \\
& =-\frac{1}{n+1} \frac{\Gamma(n+2-\varepsilon) \Gamma(1-2 \varepsilon)}{\Gamma(n+3-2 \varepsilon) \Gamma(1-\varepsilon)}\left[{ }_{2} F_{1}(-(n+1), \varepsilon-(n+1) ; 2 \varepsilon-(n+1) ; 1)-1\right] \\
& =\frac{B(n+2,-1,-2)}{(n+1)}\left[1-\frac{\Gamma(\varepsilon-(n+1)) \Gamma(n+1-\varepsilon)}{\Gamma(\varepsilon) \Gamma(-\varepsilon)}\right] .
\end{aligned}
$$

Since

$$
\frac{\Gamma(\varepsilon-(n+1))}{\Gamma(-\varepsilon)}=\frac{(-1)^{n+1} \Gamma(1-\varepsilon)}{\Gamma(n+2-\varepsilon)},
$$

then $\bar{C}_{2}^{(2)}(0, n)$ has the following form for even $n$ values

$$
\begin{aligned}
\frac{\bar{C}_{2}^{(2)}(0, n)}{N_{2} / \varepsilon^{2}} & =\frac{B(n+2,-1,-2)}{(n+1)}\left[1-\frac{(-1)^{n+1} \varepsilon}{n+1-\varepsilon}\right] \\
& =\frac{B(n+2,-1,-2)}{(n+1)} \frac{n+1-2 \varepsilon}{n+1-\varepsilon}=\frac{B(n+1,-1,-2)}{(n+1)} .
\end{aligned}
$$

The result for $\tilde{C}_{2}^{(2)}(0, n)$ can be expressed as

$$
\frac{\tilde{C}_{2}^{(2)}(0, n)}{N_{1} / \varepsilon^{2}}=\sum_{k=0}^{n} C_{n}^{k}(-1)^{k} \frac{1}{(k+1)^{2}}\left(1+\varepsilon\left[S_{1}(n-k)+\frac{2}{k+1}\right]\right)+O\left(\varepsilon^{2}\right) .
$$


The last part $C_{2}^{(4)}(0, n)$ is splitted in two parts because $S_{1}(m)=S_{1}(m+1)-1 /(m+1)$ :

$$
\begin{aligned}
& C_{2}^{(4)}(0, n)=\bar{C}_{2}^{(4)}(0, n)-\tilde{C}_{2}^{(4)}(0, n) \\
& \bar{C}_{2}^{(4)}(0, n)=\sum_{k=0}^{n}(-1)^{k} C_{N}^{k} \frac{S_{1}(k+1) S_{1}(n-k)}{k+1}, \\
& \tilde{C}_{2}^{(4)}(0, n)=\sum_{k=0}^{n}(-1)^{k} C_{N}^{k} \frac{S_{1}(n-k)}{(k+1)^{2}}
\end{aligned}
$$

It is easy to show that the part $\bar{C}_{2}^{(4)}(0, n)$ is zero at even $n$ values. Indeed,

$$
\bar{C}_{2}^{(4)}(0, n)=-\sum_{k=1}^{n+1} \frac{(-1)^{k} n ! S_{1}(k) S_{1}(n-k+1)}{k !(n-k+1) !}=-\sum_{k=0}^{n+1} \frac{(-1)^{k} n ! S_{1}(k) S_{1}(n-k+1)}{k !(n-k+1) !} .
$$

After replacement $k \rightarrow n+1-k$ the last series obtains the additional factor $(-1)^{n+1}$ and, thus, it is zero for even $n$ values. It is really the case: the results for $\bar{C}_{2}^{(4)}(0, n)$ are exactly evaluated in the part B of Appendix A.

Now we return to the coefficient function $C_{2}(0, n)$ given in Equation (69). It is convenient to split the result (64) into two parts:

$$
\frac{n+2-4 \varepsilon}{2} C_{2}(0, n)=\bar{C}_{2}(0, n)+2 \varepsilon \tilde{C}_{2}(0, n)
$$

where

$$
\begin{aligned}
& \bar{C}_{2}(0, n)=C_{2}^{(1)}(0, n)+\bar{C}_{2}^{(2)}(0, n)-C_{2}^{(3)}(0, n), \\
& \tilde{C}_{2}(0, n)=\tilde{C}_{2}^{(2)}(0, n)-\frac{\varepsilon}{2} \tilde{C}_{2}^{(4)}(0, n) .
\end{aligned}
$$

The part $\bar{C}_{2}(0, n)$ can be evaluated exactly as

$$
\bar{C}_{2}(0, n)=\frac{1}{n+1-2 \varepsilon}\left[2\left(1-\frac{\varepsilon}{n+1}\right) B(n+1,-1,-2)-B(n+1,0,-2)\right] .
$$

Using expansions (41) of $B(n+1, a, b)$ with respect of $\varepsilon$, we have

$$
\begin{aligned}
& \bar{C}_{2}(0, n)=\frac{1}{n+1-2 \varepsilon}\left[1-\frac{2 \varepsilon}{n+1}+\varepsilon^{2}\left(S_{2}(n)-S_{1}^{2}(n)-\frac{2 S_{1}(n)}{n+1}\right)\right] \\
& =\frac{1}{n+1}\left[1+\varepsilon^{2}\left(S_{2}(n)-S_{1}^{2}(n)-\frac{2 S_{1}(n)}{n+1}\right)\right] .
\end{aligned}
$$

The result for $\tilde{C}_{2}(0, n)$ can be expressed as

$$
\frac{\tilde{C}_{2}^{(2)}(0, n)}{N_{1} / \varepsilon^{2}}=\sum_{k=0}^{n} C_{n}^{k}(-1)^{k} \frac{1}{(k+1)^{2}}\left(1+\varepsilon\left[\frac{1}{2} S_{1}(n-k)+\frac{2}{k+1}\right]\right)+O\left(\varepsilon^{2}\right) .
$$

To evaluate the r.h.s it is convenient to use the result (45) for $\phi_{1}(a, n)$, to calculate the series $\Phi_{2}(a, n)$ :

$$
\Phi_{2}(a, n)=\sum_{k=0}^{n} C_{n}^{k}(-1)^{k} \frac{S_{1}(n-k)}{k+1-a}
$$


and to differentiate several times these series with respect of $a$ and put $a=0$. Indeed,

$$
\frac{\tilde{C}_{2}(0, n)}{N_{1} / \varepsilon^{2}}=\phi_{1}^{(1)}(n)+\varepsilon\left(\frac{1}{2} \Phi_{2}^{(1)}(n)+2 \phi_{1}^{(2)}(n)\right)+O\left(\varepsilon^{2}\right),
$$

where

$$
\Phi_{2}^{(m)}(n)=\left.\frac{1}{m !} \frac{\partial^{m}}{(\partial a)^{m}} \Phi_{2}(a, n)\right|_{a=0} .
$$

We consider the evaluation of the series $\Phi_{2}(a, n)$ in the next subsection.

\subsection{1. $\Phi_{2}(a, n)$}

As in the case of $\Phi_{1}(a, n)$, to obtain the results for $\Phi_{2}(a, n)$ it is convenient to consider the difference relation between $\Phi_{2}(a, n)$ and $\Phi_{2}(a, n-1)$. Indeed, we can rewrite (93) in the following way

$$
\Phi_{2}(a, n)=\sum_{k=0}^{n-1} \frac{(-1)^{k} n !}{k !(n-1-k) !} \frac{S_{1}(n-k)}{(n-k)(k+1-a)} .
$$

Taking partial fraction (48) we can represent (93) in the following way

$$
\Phi_{2}(a, n)=\frac{1}{n+1-a}\left[\sum_{k=0}^{n-1} \frac{(-1)^{k} n !}{k !(n-1-k) !} \frac{S_{1}(n-k)}{k+1-a}+\sum_{k=0}^{n-1} \frac{(-1)^{k} n !}{k !(n-k) !} S_{1}(n-k)\right] .
$$

Taking $S_{1}(n-k)=S_{1}(n-k-1)+1 /(n-k)$, we have

$$
\begin{aligned}
& \Phi_{2}(a, n)=\frac{1}{n+1-a}\left[n \Phi_{1}(a, n)+\sum_{k=0}^{n-1} \frac{(-1)^{k} n !}{k !(n-k) !} \frac{1}{k+1-a}+\sum_{k=0}^{n-1} \frac{(-1)^{k} n !}{k !(n-k) !} S_{1}(n-k)\right] \\
& =\frac{1}{n+1-a}\left[n \Phi_{1}(a, n)+\phi_{1}(a, n)-\frac{(-1)^{n}}{n+1-a}+\sum_{k=0}^{n} \frac{(-1)^{k} n !}{k !(n-k) !} S_{1}(n-k)\right] .
\end{aligned}
$$

The series in the r.h.s. can be calculated usng results for $\hat{\Phi}_{1}(\alpha, \beta)$ studied in Appendix A as

$$
\begin{aligned}
& \sum_{k=0}^{n} \frac{(-1)^{k} n !}{k !(n-k) !} S_{1}(n-k)=\left.\frac{\partial}{(\partial y)} \sum_{k=0}^{n} \frac{(-1)^{k} n ! \Gamma(1-y)}{k ! \Gamma(n-k+1-y)}\right|_{y=0} \\
& =\left.n ! \frac{\partial}{(\partial x)}\left\{\Gamma(1-y) \hat{\Phi}_{1}(\alpha=-y, \beta=1)\right\}\right|_{y=0}=\left.(-1)^{n} \frac{\partial}{(\partial x)} \frac{y}{y-n}\right|_{y=0}=-\frac{(-1)^{n}}{n} .
\end{aligned}
$$

So, we have

$$
\Phi_{2}(a, n)=\frac{1}{n+1-a}\left[n \Phi_{1}(a, n)+\frac{\Gamma(1-a) \Gamma(n+1)}{\Gamma(n+2-a)}-(-1)^{n}\left(\frac{1}{n+1-a}+\frac{1}{n}\right)\right] .
$$

It is convenient to introduce the new function $\bar{\Phi}_{2}(a, n)$ as

$$
\Phi_{2}(a, n)=\frac{\Gamma(1-a) \Gamma(n+1)}{\Gamma(n+2-a)} \bar{\Phi}_{2}(a, n),
$$

which has the following reccursion:

$$
\bar{\Phi}_{2}(a, n)=\bar{\Phi}_{2}(a, n-1)+\frac{1}{n+1-a}-(-1)^{n} \frac{\Gamma(n+1-a)}{n ! \Gamma(1-a)}\left(\frac{1}{n+1-a}+\frac{1}{n}\right) .
$$


It can be solved as

$$
\bar{\Phi}_{2}(a, n)=\bar{\Phi}_{2}(a, 0)+\sum_{m=1}^{n}\left[\frac{1}{m+1-a}-(-1)^{m} \frac{\Gamma(m+1-a)}{m ! \Gamma(1-a)}\left(\frac{1}{m+1-a}+\frac{1}{m}\right)\right] .
$$

Since $\Phi_{2}(a, n=0)=0$, then $\bar{\Phi}_{2}(a, 0)=0$ and we find finally

$$
\Phi_{2}(a, n)=\frac{\Gamma(1-a) \Gamma(n+1)}{\Gamma(n+2-a)} \sum_{m=1}^{n}\left[\frac{1}{m+1-a}-(-1)^{m} \frac{\Gamma(m+1-a)}{m ! \Gamma(1-a)}\left(\frac{1}{m+1-a}+\frac{1}{m}\right)\right]
$$

and for $a=0$

$$
\Phi_{2}(0, n)=\frac{1}{n+1} \sum_{m=1}^{n+1}\left[\frac{1}{m+1}-(-1)^{m}\left(\frac{1}{m+1}+\frac{1}{m}\right)\right]=\frac{1}{n+1}\left(S_{1}(n+1)+\frac{(-1)^{n+1}}{n+1}\right) .
$$

We can calculate $\Phi_{2}(0, n)$ directly in the way similar to the evaluation of $\Phi_{1}(0, n)$. The direct calculation is a full agreement of such calculations with (102).

We have for $\Phi_{2}^{(1)}(n)$ :

$$
\begin{aligned}
& \Phi_{2}^{(1)}(n)=-\frac{S_{1}(n+1)}{n+1} \sum_{m=1}^{n+1}\left[\frac{1}{m+1}-(-1)^{m}\left(\frac{1}{m+1}+\frac{1}{m}\right)\right] \\
& +\frac{1}{n+1} \sum_{m=1}^{n+1}\left[\frac{1}{(m+1)^{2}}-(-1)^{m} S_{1}(m)\left(\frac{1}{m+1}+\frac{1}{m}\right)\right] .
\end{aligned}
$$

After little algebra, we obtain the final resuls

$$
\Phi_{2}^{(1)}(n)=\frac{1}{n+1}\left(S_{2}(n+1)+2 S_{-2}(n+1)+S_{1}^{2}(n+1)\right) .
$$

We would like to note about an appearence the sum $S_{-2}(n)$, which cannot be obtained from expansions of products of $\Gamma$-functions.

Taking the results for $\phi_{1}^{(1)}(n), \phi_{1}^{(2)}(n)$ and $\Phi_{2}^{(2)}(n)$ together, we can obtain the following results for $\tilde{C}_{2}(0, n)$

$$
\frac{\tilde{C}_{2}(0, n)}{N_{1} / \varepsilon^{2}}=\frac{1}{n+1-2 \varepsilon}\left[S_{1}(n)+\frac{1}{n+1}+\varepsilon\left(\frac{3}{2} S_{1}^{2}(n)+\frac{3}{2} S_{2}(n)+S_{-2}(n)+\frac{S_{1}(n)}{n+1}\right)\right]+O\left(\varepsilon^{2}\right) .
$$

3.3.2. $C_{2}(0, n)$

Taking the results for $\bar{C}_{2}(0, n)$ and $\tilde{C}_{2}(0, n)$ together, we have for $C_{2}(0, n)$

$$
C_{2}(0, n)=\frac{2 N_{1}}{(n+2-4 \varepsilon)(n+1-2 \varepsilon)}\left[\frac{1}{\varepsilon^{2}}+\frac{2}{\varepsilon} S_{1}(n)+2 S_{1}^{2}(n)+4 S_{2}(n)+2 S_{-2}(n)\right] \text {. }
$$


Changing the nested sums $S_{i}(n)$ to $S_{i}(n+1)$, we can simplify these results:

$$
\begin{aligned}
C_{2}(0, n)= & \frac{2 N_{1}}{(n+2-4 \varepsilon)(n+1)}\left[\frac{1}{\varepsilon^{2}}+\frac{2}{\varepsilon} S_{1}(n+1)\right. \\
& \left.+2 S_{1}^{2}(n+1)+4 S_{2}(n+1)+2 S_{-2}(n+1)\right] \\
= & \frac{2 N_{1}}{(n+2)(n+1)}\left[\frac{1}{\varepsilon^{2}}+\frac{2}{\varepsilon}\left(S_{1}(n+1)+\frac{1}{n+2}\right)\right. \\
& \left.+2 S_{1}^{2}(n+1)+4 S_{2}(n+1)+2 S_{-2}(n+1)+8 \frac{S_{1}(n+1)}{n+2}+\frac{16}{(n+2)^{2}}\right] .
\end{aligned}
$$

We note, that we can put the normalization in the definition of $\mu_{g}^{2}$-scale of $g$-scheme [129], which relates with the usual $\overline{M S}$ one as $\mu_{g}^{2}=K_{1} \mu_{\overline{M S}}^{2}$.

Using the fact that the above result (107) is obtained for even $n$ values (and $C_{2}(0,2 m+1)=0$ ), we can recover the full resul for $C_{2}(0, n)$ in the following form:

$$
\begin{aligned}
C_{2}(0, n)= & \frac{\left(1+(-1)^{n}\right) N_{1}}{(n+2)(n+1)}\left[\frac{1}{\varepsilon^{2}}+\frac{2}{\varepsilon}\left(S_{1}(n+1)+\frac{1}{n+2}\right)\right. \\
& \left.+2 S_{1}^{2}(n+1)+4 S_{2}(n+1)+2 S_{-2}(n+1)+8 \frac{S_{1}(n+1)}{n+2}+\frac{16}{(n+2)^{2}}\right] .
\end{aligned}
$$

3.4. $I_{1}(1, n)$ and $I_{2}(1, n)$

Now we evaluate the particular cases $I_{1}(1, n)$ and $I_{2}(1, n)$, which are important for the future studies. Indeed, we will see that the integral are finite and have very compact form.

The results for the $C_{1,1}(1, n)$ and $C_{2,1}(1, n)$ are

$$
\begin{aligned}
& C_{1,1}(1, n)=-\frac{N_{2}}{2 \varepsilon^{2}}\left[\frac{2 K_{1}}{K_{2}} \frac{B(n+1,-1,-2)}{n+1-2 \varepsilon}-\frac{B(n+1,-2,-3)}{n+1-3 \varepsilon}\right], \\
& C_{2,1}(1, n)=-\frac{N_{2}}{2 \varepsilon^{2}} \frac{B(n+1,-1,-3)}{n+1-2 \varepsilon},
\end{aligned}
$$

where the normalization factors $N_{1}$ and $N_{2}$ and also the factors $K_{1}$ and $K_{2}$ are given in Eqsuations (63) and (36), respectively.

Below we consider the results for $I_{1}(1, n)$ and $I_{2}(1, n)$ is the different subsections.

\subsection{1. $I_{1}(1, n)$}

Following to the case $C_{2}(0, n)$ (see Equation (85)) it is convenient to split the result for $C_{2}(1, n)$ into tow parts

$$
C_{1}(1, n)=\bar{C}_{1}(1, n)+2 \varepsilon \tilde{C}_{1}(0, n),
$$

i.e., we take together the coefficient functions $C_{1,1}(1, n)$ and $C_{2,1}(1, n)$ and also the function $\bar{C}_{1}(0, n)$ of Equation (37) and to denote this contributon as $\bar{C}_{1}(1, n)$. So, we have

$$
\bar{C}_{1}(1, n)=\bar{C}_{1}(0, n)-\frac{1}{2 \varepsilon}\left(2 C_{1,1}(1, n)-C_{2,1}(1, n)\right) .
$$


Taking the results (109), (110) and (37) we have for $\bar{C}_{1}(1, n)$

$$
\begin{aligned}
\frac{\bar{C}_{1}(1, n)}{N_{2} /\left(2 \varepsilon^{2}\right)}= & \frac{1}{\varepsilon}\left[\frac{2 K_{1}}{K_{2}} \frac{B(n+1,-1,-2)}{n+1-2 \varepsilon}\right. \\
& \left.-\frac{B(n+1,-1,-3)}{n+1-2 \varepsilon}-\frac{2 \varepsilon B(n+1,-2,-3)}{(n+1)(n+1-3 \varepsilon)}-\frac{1}{n+1}\right] .
\end{aligned}
$$

Expanding the r.h.s. with

$$
\frac{K_{1}}{K_{2}}=\exp \left[6 \zeta_{3} \varepsilon^{3}\right]
$$

we have

$$
\left.\frac{\bar{C}_{1}(1, n)}{N_{2} /\left(2 \varepsilon^{2}\right)}=\frac{1}{n+1-2 \varepsilon}\left[-\hat{A}(n) \varepsilon+\left(12 \zeta_{3}-\hat{B}(n)\right) \varepsilon^{2}+\right)\left(\varepsilon^{3}\right)\right]+O\left(\varepsilon^{3}\right),
$$

where

$$
\begin{aligned}
& \hat{A}(n)=S_{1}^{2}(n)+S_{2}(n)+\frac{2 S_{1}(n)}{n+1}+\frac{2}{(n+1)^{2}}=\hat{C}(n) \\
& \hat{B}(n)=S_{1}^{3}(n)+5 S_{1}(n) S_{2}(n)+4 S_{3}(n)+\frac{1}{n+1}\left(S_{1}^{2}(n)+5 S_{2}(n)\right)+\frac{2 S_{1}(n)}{(n+1)^{2}}+\frac{6}{(n+1)^{3}}
\end{aligned}
$$

and we see that $\hat{A}(n)$ is equal to $\hat{C}(n)$ in (58).

Taking together the results (61) and (115), we obtain finally

$$
C_{1}(1, n)=\frac{1}{n+1}\left(S_{3}(n)+S_{1}(n) S_{2}(n)-S_{2,1}(n)+6 \zeta_{3}\right)+O(\varepsilon) .
$$

3.4.2. $I_{2}(1, n)$

Consider the contribution of $C_{2}(0, n)$ to $C_{2}(1, n)$. It has the form (see Eq.(16) for $\alpha=1$ )

$$
-\frac{n+2 d-6}{n+d-4} C_{2}(0, n)=-\frac{n+2-4 \varepsilon}{n-2 \varepsilon} C_{2}(0, n),
$$

where $C_{2}(0, n)$ is given above in (108). So, we have

$$
-\frac{n+2-4 \varepsilon}{n-2 \varepsilon} C_{2}(0, n)=-\frac{2 N_{1}}{(n-2 \varepsilon)(n+1-2 \varepsilon)}\left[\frac{1}{\varepsilon^{2}}+\frac{2}{\varepsilon} S_{1}(n)+2 S_{1}^{2}(n)+4 S_{2}(n)+2 S_{-2}(n)\right] .
$$

Using the result for $C_{21}(1, n)$ of $(110)$, we obtain the second contribution to $C_{2}(1, n)$ as

$$
-\frac{2}{n-2 \varepsilon} C_{21}(1, n)=\frac{2 N_{1}}{(n-2 \varepsilon)(n+1-2 \varepsilon)}\left[\frac{1}{\varepsilon^{2}}+\frac{2}{\varepsilon} S_{1}(n)+2 S_{1}^{2}(n)+4 S_{2}(n)\right] .
$$

Since $K_{1} / K_{2} \sim O\left(\varepsilon^{3}\right)$ (see Equation (114)) and, thus, $N_{1} / N_{2} \sim O\left(\varepsilon^{3}\right)$. So, the difference between the normalizations $N_{1}$ and $N_{2}$ in negligible for Equations (120) and (121). Taking these two results together, we have for $C_{2}(1, n)$

$$
C_{2}(1, n)=-\frac{4 S_{-2}(n)}{n(n+1)}+O(\varepsilon) .
$$

The results is convenient for $n \geq 1$ (really the result $C_{1}(1, n=0)=6 \zeta_{3}$ can be obtained directly from Equation (122) by using an analytic continuations of the sum $S_{-2}(n)$ (see Appendix C)). For the case $n=0$ we can use the results for $C_{1}(1, n=0)=6 \zeta_{3}$, because $C_{2}(1, n=0)=C_{1}(1, n=0)$. 
Using the fact that the above result (121) is obtained for even $n$ values (and $C_{2}(1,2 m+1)=0$ ), we can recover the full resul for $C_{2}(1, n)$ in the following form

$$
C_{2}(1, n)=\left(1+(-1)^{n}\right)\left(3 \delta_{n}^{0} \zeta_{3}-\left(1-\delta_{n}^{0}\right) \frac{2 S_{-2}(n)}{n(n+1)}\right) .
$$

\section{Examples of Calculation of Some Type of Massless Diagrams}

In the previous section, we studied the basic diagrams $I_{1}(\alpha, n)$ and $I_{1}(\alpha, n)$ with $\alpha=0$ and $\alpha=1$. I would especially like to note the case $\alpha=1$ where the coefficient functions of the integrals $I_{1}(1, n)$ and $I_{1}(1, n)$ have a very compact form.

In this section, we consider the expansion coefficients of scalar diagrams (we call them the "moments" of diagrams), arising in the study of forward elastic scattering. These moments are extracted from the initial diagrams by the method of "projectors" [20,21]. Some basic diagrams and getting their points are discussed in Appendix B.

Here we look at the diagrams $I_{1}(\alpha, n)$ and $I_{1}(\alpha, n)$ with $\alpha=n+1$ and $\alpha=n+2$.

4.1. $I_{1}(n+1, n)$ and $I_{2}(n+1, n)$

Let us first consider the two simplest diagrams: $J_{1}(\alpha=1, q, p)$ and $J_{2}(\alpha=1, q, p)$, shown in Figure 2. As it was discussed already, using the method of "projectors" (see Appendix B) we can study the so-called moments $J_{i}(\alpha=1, n)(i=1,2)$. The moments of the diagrams shown in Figure 2 are represented by the diagrams shown in Equation (180) for $\alpha=n+1$, i.e., $J_{i}(\alpha=1, n)=I_{i}(n+1, n)$.

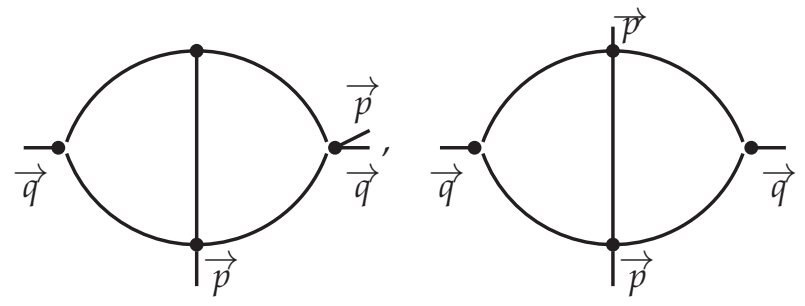

Figure 2. Most simplest diagrams: $J_{1}(\alpha=1, q, p)$ and $J_{2}(\alpha=1, q, p)$.

To evaluate the diagrams it is convenient to use the so-called momentum transformation. To show it's effectivity it is useful to consider more complicated diagrams $I_{i}(\alpha+n, n)(i=1,2)$, which have the coefficient functions $C_{i}(\alpha+n, n)$

$$
I_{i}(\alpha+n, n)=\frac{1}{(4 \pi)^{d}} C_{i}(\alpha+n, n) \frac{q^{\mu_{1} \ldots \mu_{n}}}{q^{2(\alpha+n+2 \varepsilon)}},
$$

which is similar to (19).

Now we introduce the following Fourier transforms [16]

$$
\begin{aligned}
& \int d^{d} p e^{i p x} \frac{1}{p^{2 \alpha}}=2^{2 \tilde{\alpha}} \pi^{d / 2} a_{0}(\alpha) \frac{1}{x^{2 \tilde{\alpha}}},\left(\tilde{\alpha}=\frac{d}{2}-\alpha, \text { see Equation (6)) },\right. \\
& \int d^{d} p e^{i p x} \frac{p^{\mu_{1}} \ldots p^{\mu_{n}}}{p^{2(\alpha+n)}}=(-i)^{n} 2^{2 \tilde{\alpha}} \pi^{d / 2} a_{n}(\alpha+n) \frac{p^{\mu_{1}} \ldots p^{\mu_{n}}}{x^{2 \tilde{\alpha}}}+\ldots,
\end{aligned}
$$

where we neglect the terms of the order $g^{\mu_{i} \mu_{j}}$.

The Fourier transform (126) is the usual one (see, for example, the recent review [16]) and the Fourier transform (126) can be obtained from (126) with the help of projector $\partial^{\mu_{1}} /(\partial x)^{\mu_{1}} \ldots \partial^{\mu_{n}} /(\partial x)^{\mu_{n}}$.

Applijing the Fourier transforms to 1.h.s. we will come to the following diagrams in $x$-space: 

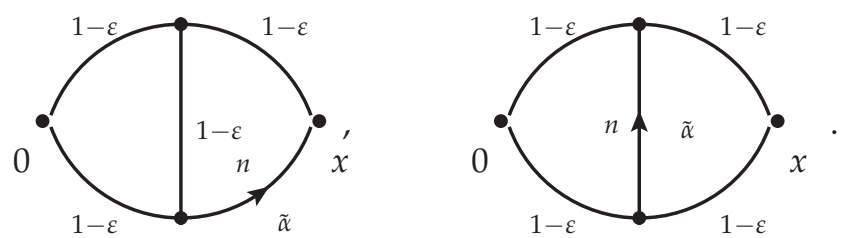

If we replace $x$ and all internal coordinats by the corresponding moment $q$ and the internal moments we obtain the corresponding diagram in the momentum space. We will call them as $\bar{I}_{i}(\tilde{\alpha}, n)$. Such procedure is called by "dual transformation" (see discussions in Ref. [16]) and denoted as $\stackrel{d}{=}$ (see aslo Introduction). Therefore, we have
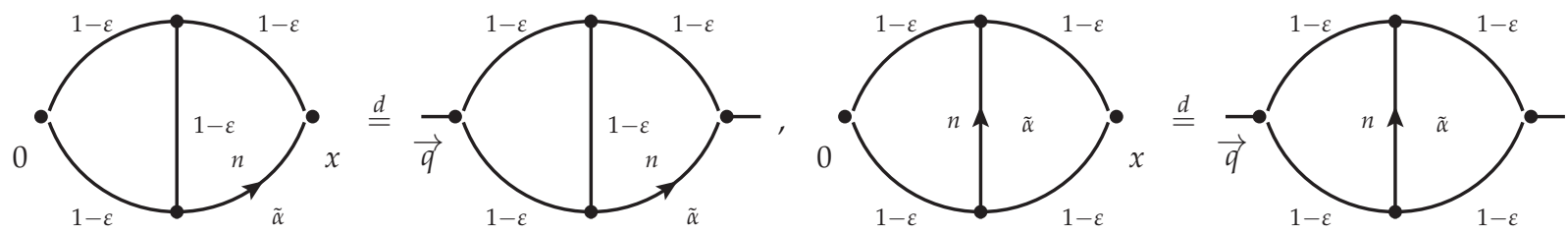

Lets to denote the coefficient functions of the last integral as $\bar{C}_{i}(\tilde{\alpha}, n)$, i.e.

$$
\bar{I}_{i}(\tilde{\alpha}, n)=\frac{1}{(4 \pi)^{d}} \bar{C}_{i}(\tilde{\alpha}, n) \frac{q^{\mu_{1} \ldots \mu_{n}}}{q^{2(\tilde{\alpha}-2 \varepsilon)}} .
$$

Taking the Fourier transforms for the both side of Equation (124) (see Ref. [16]), we obtain the relations between the coefficient functions $C_{i}(\alpha+n, n)$ and $\bar{C}_{i}(\tilde{\alpha}, n)$

$$
C_{i}(\alpha+n, n)=K(\alpha+n, n) \bar{C}_{i}(\tilde{\alpha}, n),
$$

where

$$
K(\alpha+n, n)=\frac{a_{0}^{4}(1) a_{n}(\alpha+n)}{a_{n}(\alpha+n+2 \varepsilon)}=\frac{\Gamma^{4}(1-\varepsilon) \Gamma(\tilde{\alpha}) \Gamma(\alpha+n+2 \varepsilon)}{\Gamma(\alpha+n) \Gamma(\tilde{\alpha}-2 \varepsilon)} .
$$

Now return to the case $\alpha=1$. With the accuracy $O(\varepsilon)$,

$$
\bar{C}_{i}(1-\varepsilon, n) \approx C_{i}(1, n), K(n+1, n)=\frac{\Gamma^{5}(1-\varepsilon) \Gamma(n+1+2 \varepsilon)}{n ! \Gamma(1-3 \varepsilon)} \approx 1,
$$

where the sign $\approx$ means equality up to $0\left(\varepsilon^{0}\right)$.

Thus, we have from (131)

$$
C_{i}(n+1, n)=C_{i}(1, n)+O(\varepsilon),
$$

i.e.,

$$
\begin{aligned}
& C_{1}(n+1, n)=\frac{1}{n+1}\left(S_{3}(n)+S_{1}(n) S_{2}(n)-S_{2,1}(n)+6 \zeta_{3}\right)+O(\varepsilon), \\
& C_{2}(n+1, n)=\left(1+(-1)^{n}\right)\left(3 \delta_{n}^{0} \zeta_{3}-\left(1-\delta_{n}^{0}\right) \frac{2 S_{-2}(n)}{n(n+1)}+O(\varepsilon)\right) .
\end{aligned}
$$

4.2. $I_{1}(n+2, n)$ and $I_{2}(n+2, n)$

Consider the more complex diagrams $\bar{J}_{1}(\alpha=1, q, p)$ and $\bar{J}_{2}(\alpha=1, q, p)$ shown in Figure 3. 

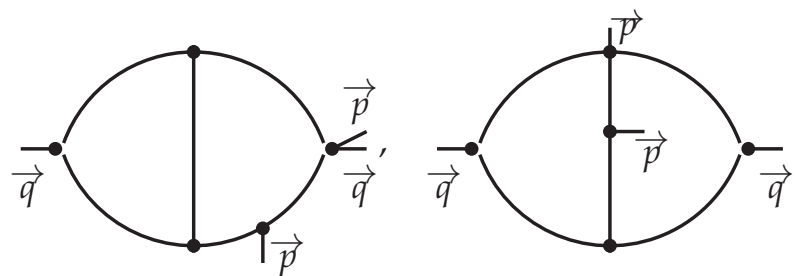

Figure 3. The diagrams $\bar{J}_{1}(\alpha=1, q, p)$ and $\bar{J}_{2}(\alpha=1, q, p)$.

Their moments $\bar{J}_{i}(\alpha=1, n)(i=1,2)$ are represented by the diagrams in Equation (180) with $\alpha=n+2$, i.e., by the diagrams $I_{i}(n+2, n)$. Their coefficient functions $C_{i}(n+2, n)(i=1,2)$ can be expressed throught the ones $C_{i}(n+1, n)$ given in Equations (132) and (133) and $C_{i 1}(n+2, n)$ given in Equations (21) and (22) at $\alpha=n+2$.

Performing calculations, we have the results for the $C_{11}(1, n)$ and $C_{21}(1, n)$ are

$$
\begin{aligned}
& \frac{C_{11}(n+2, n)}{N_{2} /\left(2 \varepsilon^{2}\right)}=\frac{2 K_{1}}{K_{2}} \frac{B(n+1,1,0)}{n+1}-\frac{B(n+1,2,1)}{n+1+\varepsilon}=\frac{2 K_{1}}{K_{2}} \frac{B(n+2,1,0)}{n+1+\varepsilon}-\frac{B(n+2,2,1)}{n+1+2 \varepsilon}, \\
& \frac{C_{2,1}(n+2, n)}{N_{2} /\left(2 \varepsilon^{2}\right)}=\frac{B(n+1,1,0)}{n+1+\varepsilon} B(n+1,2,1)-3 \frac{B(n+1,1,0)}{n+1} B(n+2,2,1),
\end{aligned}
$$

where the normalization factors $N_{1}$ and $N_{2}$ and also the factors $K_{1}$ and $K_{2}$ are given in Equations (63) and (36), respectively.

Since

$$
B(n+2,2,1)=B(n+2,2,1) \frac{n+1+2 \varepsilon}{n+1+\varepsilon} \text { and } B(n+1,1,0) B(n+1,2,1)=B(n+1,2,0),
$$

then

$$
\frac{C_{2,1}(n+2, n)}{N_{2} /\left(2 \varepsilon^{2}\right)}=-2 \frac{B(n+1,1,0)}{n+1+\varepsilon} \frac{n+1+3 \varepsilon}{n+1}=-2 \frac{B(n+2,1,0)}{n+1+\varepsilon} \frac{n+1+3 \varepsilon}{n+1+2 \varepsilon} .
$$

Expanding all $B(n+2, \ldots)$, we have

$$
\begin{aligned}
& (n+1) \frac{C_{11}(n+2, n)}{N_{2} /\left(2 \varepsilon^{2}\right)}=1+\varepsilon S_{1}(n+1)+\varepsilon^{2}\left[\frac{1}{2}\left(S_{1}^{2}(n+1)+S_{2}(n+1)\right)-\frac{1}{(n+1)^{2}}\right] \\
& +\varepsilon^{3}\left[\frac{1}{6}\left(S_{1}^{3}(n+1)+3 S_{1}(n+1) S_{2}(n+1)-10 S_{3}(n+1)\right)+12 \zeta_{3}-\frac{2 S_{2}(n+1)}{n+1}\right. \\
& \left.-\frac{2 S_{1}(n+1)}{(n+1)^{2}}+\frac{6}{(n+1)^{3}}\right]+O\left(\varepsilon^{4}\right), \\
& (n+1) \frac{C_{21}(n+2, n)}{N_{2} /\left(2 \varepsilon^{2}\right)}=-2\left(1+2 \varepsilon S_{1}(n+1)+2 \varepsilon^{2}\left[S_{1}^{2}(n+1)-S_{2}(n+1)-\frac{1}{(n+1)^{2}}\right]\right. \\
& \left.+2 \varepsilon^{3}\left[\frac{2}{3}\left(S_{1}^{3}(n+1)-3 S_{1}(n+1) S_{2}(n+1)+2 S_{3}(n+1)\right)-\frac{2 S_{1}(n+1)}{(n+1)^{2}}+\frac{3}{(n+1)^{3}}\right]\right) \\
& +O\left(\varepsilon^{4}\right) .
\end{aligned}
$$


Taking the results (138) and (139) together with the results for $C_{i}(n+1, n)(i=1,2)$ in Equations (132) and (133), we have the final results

$$
\begin{aligned}
& (n+1) \frac{C_{1}(n+2, n)}{N_{2}}=\frac{1}{2 \varepsilon^{2}} S_{1}(n+1)+\frac{1}{4 \varepsilon}\left[3 S_{1}^{2}(n+1)-5 S_{2}(n+1)\right]+\frac{7}{12} S_{1}^{3}(n+1) \\
& -\frac{5}{4} S_{1}(n+1) S_{2}(n+1)+\frac{19}{6} S_{3}(n+1)-2 S_{2,1}(n+1)-\frac{S_{1}(n+1)}{(n+1)^{2}} \\
& (n+1) \frac{C_{2}(n+2, n)}{N_{2}}=\frac{1+(-1)^{n}}{n+2}\left(\frac{1}{\varepsilon^{2}}+\frac{2}{\varepsilon}\left[S_{1}(n+1)-\frac{1}{n+2}\right]\right. \\
& \left.+2 S_{1}^{2}(n+1)-2 S_{2}(n+1)-4 \frac{S_{1}(n+1)}{n+2}+\frac{1}{(n+2)^{2}}\right),
\end{aligned}
$$

where we added the additional factor $\left(1+(-1)^{n}\right) / 2$ to the coefficient function $C_{2}(n+2, n)$.

Consideration of the more complicated examples can be found in Refs. [24,26].

We only note here that for diagrams containing several propagators depending on the momentum $p$ (see, for example, $\hat{J}_{1}(\alpha, \beta, q, p)$ in Appendix B), their moments will contain the sum of two-point diagrams (see the moment $\hat{J}_{1}(\alpha, \beta, n)$ in Appendix B).

Calculation of moments of this type is a much more serious problem compared to $I_{i}(n+1, n)$ and $I_{i}(n+2, n)$ with $(i=1,2)$. Nevertheless, as was shown in Ref. [26], it is almost always possible to separate the contributions of complex integrals and complex sums. Moreover, $\varepsilon$-singularities remain only in the simplest parts, where it is usually possible to sum in all orders in $\varepsilon$.

\section{Calculation of Massive Feynman Integrals}

Feynman integrals with massive propagators are significantly more complex objects compared to the massless case. The basic rules for calculating such diagrams are discussed in Section 2, which are supplemented by new ones containing directly massive propagators. These additional rules are discussed in the next subsection.

\subsection{Basic Formulas}

Let us briefly consider the rules for calculating diagrams with the massive propagators.

Propagator with mass $M$ will be represented as

$$
\frac{1}{\left(q^{2}+M^{2}\right)^{\alpha}}=\underset{\vec{q}}{\underset{\sim}{\sim}} \frac{M}{\alpha}
$$

The following formulas hold.

A. For simple chain of two massive propagators with the same mass:

or graphically

$$
\frac{1}{\left(q^{2}+M^{2}\right)^{\alpha_{1}}} \frac{1}{\left(q^{2}+M^{2}\right)^{\alpha_{2}}}=\frac{1}{\left(q^{2}+M^{2}\right)^{\left(\alpha_{1}+\alpha_{2}\right)}},
$$

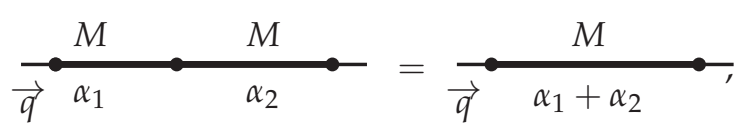

i.e., the product of propagators with the same mass $M$ is equivalent to a new propagator with the mass $M$ and an index equal to the sum of the indices of the original propagators. 
B. Massive tadpole is integrated as

$$
\int \frac{D k}{k^{2 \alpha_{1}}\left(k^{2}+M^{2}\right)^{\alpha_{2}}}=\frac{1}{(4 \pi)^{d / 2}} \frac{R\left(\alpha_{1}, \alpha_{2}\right)}{M^{2\left(\alpha_{1}+\alpha_{2}-d / 2\right)}},
$$

where

$$
R(\alpha, \beta)=\frac{\Gamma\left(d / 2-\alpha_{1}\right) \Gamma\left(\alpha_{1}+\alpha_{2}-d / 2\right)}{\Gamma(d / 2) \Gamma\left(\alpha_{2}\right)}
$$

C. A simple loop of two massive propagators with masses $M_{1}$ and $M_{1}$ can be represented as hypergeometric function, which can be calculated in a general form, for example, by Feynman-parameter method. It is very conveninet, using this approach to represent the loop as an integral of a propagator with the "effective mass" $\mu[56,132-136]$ :

$$
\begin{aligned}
& (4 \pi)^{d / 2} \times \int \frac{D k}{\left[(q-k)^{2}+M_{1}^{2}\right]^{\alpha_{1}\left[k^{2}+M_{2}^{2}\right]^{\alpha_{2}}}} \\
& =\frac{\Gamma\left(\alpha_{1}+\alpha_{2}-d / 2\right)}{\Gamma\left(\alpha_{1}\right) \Gamma\left(\alpha_{2}\right)} \int_{0}^{1} \frac{d s s^{\alpha_{1}-1}(1-s)^{\alpha_{2}-1}}{\left[s(1-s) q^{2}+M_{1}^{2} s+M_{2}^{2}(1-s)\right]^{\alpha_{1}+\alpha_{2}-d / 2}} \\
& =\frac{\Gamma\left(\alpha_{1}+\alpha_{2}-d / 2\right)}{\Gamma\left(\alpha_{1}\right) \Gamma\left(\alpha_{2}\right)} \int_{0}^{1} \frac{d s}{s^{1-\tilde{\alpha}_{2}}(1-s)^{1-\tilde{\alpha}_{1}}} \frac{1}{\left[q^{2}+\mu^{2}\right]^{\alpha_{1}+\alpha_{2}-d / 2}}, \quad\left(\mu^{2}=\frac{M_{1}^{2}}{1-s}+\frac{M_{2}^{2}}{s}\right) .
\end{aligned}
$$

It is useful to rewrite the equation graphically as:

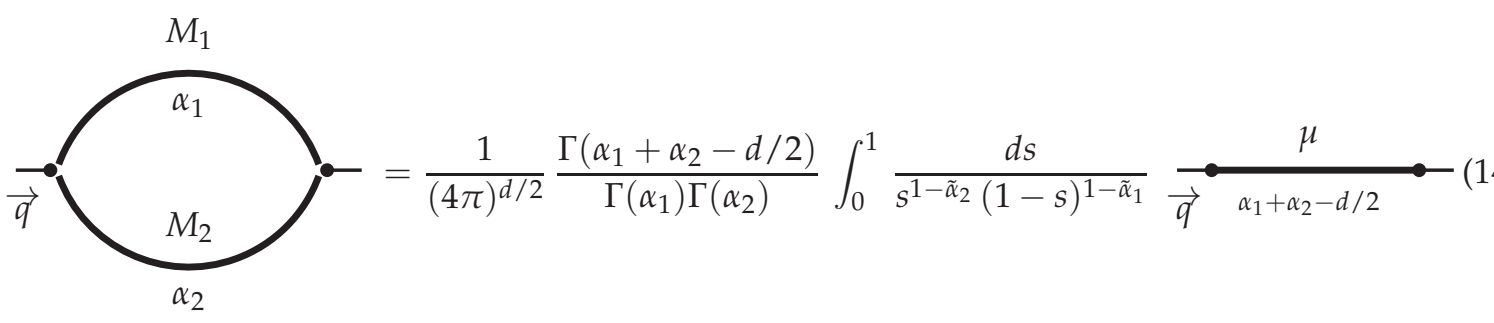

D. For any triangle with indices $\alpha_{i}(i=1,2,3)$ and masses $M_{i}$ there is the following relation, which is based on integration by parts (IBP) procedure $[7,123,124,136-138]$

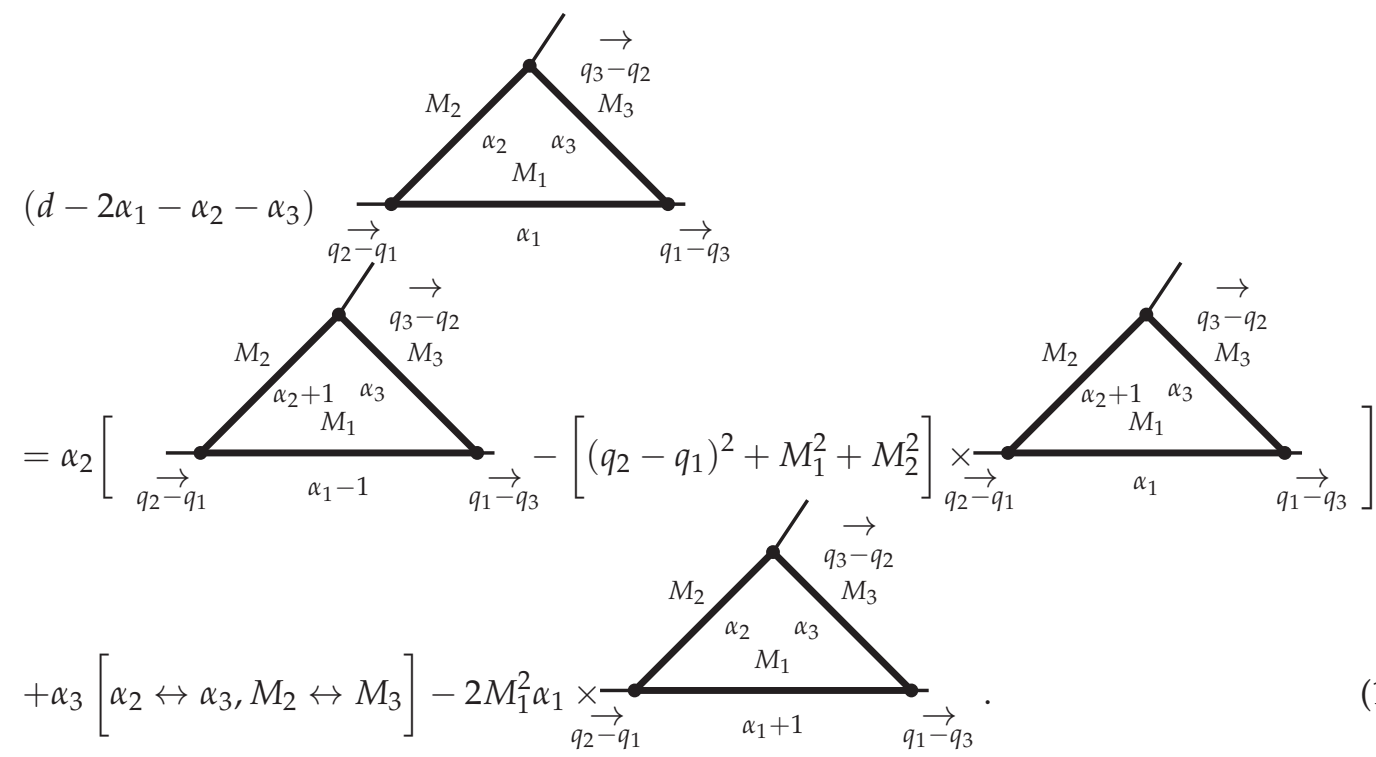


As it was in the massless case (see Equation (12)), Equation (146) can been obtained by introducing the factor $\left(\partial / \partial k_{\mu}\right)\left(k-q_{1}\right)^{\mu}$ to the subintegral expression of the triangle and using the integration by parts procedure as in Equation (13).

As it was in the massless case, the line with the index $\alpha_{1}$ is distingulished. The contributions of the other lines are same. So, we will call below the line with the index $\alpha_{1}$ as a "distingulished line". It is clear that a various choices of the distingulished line produce different tipes of the IBP relations.

\subsection{Basic Massive Two-Loop Integrals}

The general topology of the two-loop two-point diagram, which cannot be expressed as a combination of loops and chanins is shown on Figure 4.
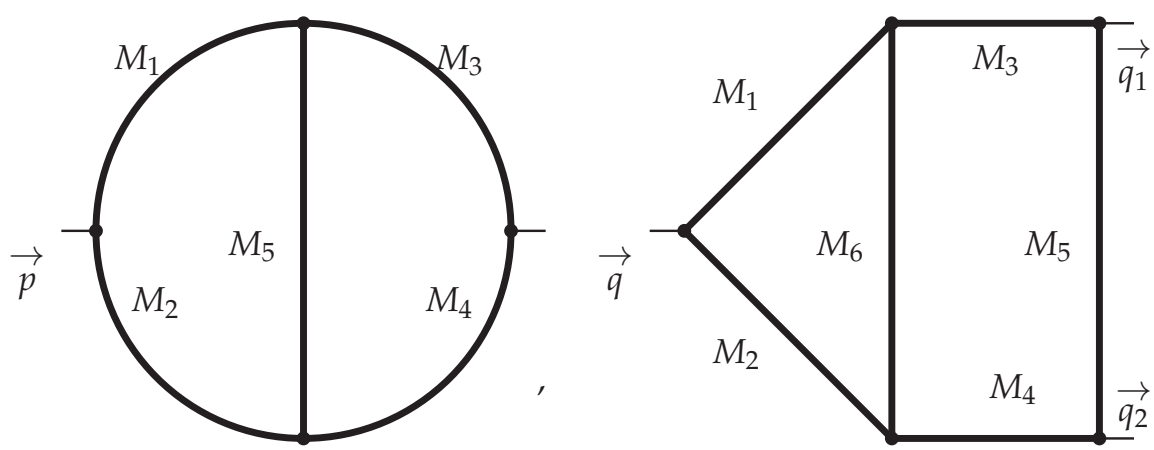

Figure 4. Two-loop two-point diagram $I\left(M_{1}, M_{2}, M_{3}, M_{4}, M_{5}\right)$ and three-point diagram $P\left(M_{1}, M_{2}, M_{3}, M_{4}, M_{5}, M_{6}\right)$ with $q_{1}^{2}=q_{2}^{2}=0$.

Below in the present analysis we will concentrate mostly on two-loop two-point and three-point diagrams, which can be taken from the diagram shown in Figure 4. We will call them as:

$$
\begin{aligned}
& I_{j}=I\left(M_{j}=M \neq 0, M_{p}=0, p \neq j\right), \quad I_{i j}=I\left(M_{i}=M_{j}=M \neq 0, M_{p}=0, p \neq i \neq j\right), \\
& I_{i j s}=I\left(M_{i}=M_{j}=M_{s}=M \neq 0, M_{p}=0, p \neq i \neq j \neq s\right), \\
& I_{i j s t}=I\left(M_{i}=M_{j}=M_{s}=M_{t}=M \neq 0, M_{p}=0, p \neq i \neq j \neq s \neq t\right), \\
& P_{j}=P\left(M_{j}=M \neq 0, M_{p}=0, p \neq j\right), P_{i j}=P\left(M_{i}=M_{j}=M \neq 0, M_{p}=0, p \neq i \neq j\right), \\
& P_{i j s}=P\left(M_{i}=M_{j}=M_{s}=M \neq 0, M_{p}=0, p \neq i \neq j \neq s\right), \\
& P_{i j s t}=P\left(M_{i}=M_{j}=M_{s}=M_{t}=M \neq 0, M_{p}=0, p \neq i \neq j \neq s \neq t\right) .
\end{aligned}
$$

Application of the IBP procedure $[123,124]$ to loop internal momenta leads to relations between various Feynman integrals and, therefore, to the necessity of calculating only some of them, which in a sense are independent. These independent diagrams (which were chosen completely arbitrarily, of course) are called master integrals [55].

Applying the IBP procedure $[123,124]$ to the master-integrals themselves leads to differential equations [28,29,136-140] for them with the inhomogeneous terms containing less complex diagrams (the "less complex diagrams" usually contain less number of propagators and sometimes they can be represented as diagrams with less number of loops and with some "effective masses" (see, for example, [56,132-135,141] and references therein)). Applying the IBP procedure to diagrams in inhomogeneous terms leads to new differential equations for them with new inhomogeneous terms containing even more less complex diagrams ( $\equiv$ less $^{2}$ complex ones). By repeating the procedure several times, in the last step we can obtain inhomogeneous terms containing mainly tadpoles, which can be easily calculated in-turn (see also the discussions in Section 7 below).

Solving the corresponding differential equations in this last step, diagrams for the inhomogeneous terms of the differential equations in the previous step can be reproduced. Repeating the procedure several times, me can get the results for the original Feynman diagram. 


\section{Evaluation of Series}

As the first example, we consider P integral, whous graphical representation has the following form

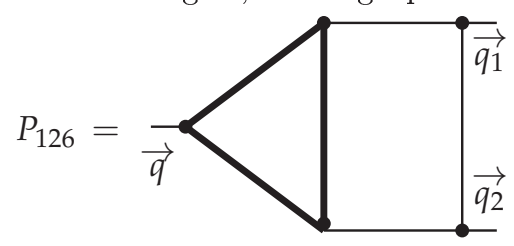

This integral was calculated in Ref. [142] and presented also in $[56,143]$.

It has the following series representation (In Minkowski space all $\ln ^{n} x \rightarrow \ln ^{n}(-x)$ and there is an imaginary part which is important for cuts of the diagram. Such property exists also in other examples (see Section 6.3 below)):

$$
\begin{aligned}
P_{125}= & -\frac{\hat{N}}{\left(q^{2}\right)^{2}} \sum_{n=1} \frac{(-x)^{n}}{2 n}\left[\frac { ( n ! ) ^ { 2 } } { ( 2 n ) ! } \left\{\frac{1}{\varepsilon^{2}}-\frac{1}{\varepsilon}\left(S_{1}+\ln x\right)+5 \bar{S}_{1} S_{1}-\frac{1}{2}\left(\bar{S}_{1}^{2}+15 S_{1}^{2}+S_{2}\right)\right.\right. \\
& \left.\left.+\frac{2}{n} S_{1}-S_{1} \ln x+\frac{1}{2} \ln ^{2} x\right\}+n F(n)\right], \quad\left(x=\frac{q^{2}}{M^{2}}\right),
\end{aligned}
$$

where

$$
S_{i}=S_{i}(n-1), \bar{S}_{i}=S_{i}(n-1) .
$$

Here the normalization $\hat{N}=\left(\bar{\mu}^{2} / m^{2}\right)^{2 \varepsilon}$, where $\bar{\mu}=4 \pi e^{-\gamma_{E}} \mu$ is in the standard $\overline{M S}$-scheme and $\gamma_{E}$ is the Euler constant.

Equation (149) contains the function $F(n)$ :

$$
F(n)=F_{1}(n)-F_{2}(n)+F_{3}(n)-F_{4}(n),
$$

with

$$
\begin{aligned}
& F_{1}(n)=\sum_{m=1}^{n-1} \frac{\Gamma(n) \Gamma(n-m)}{m \Gamma(2 n-m)}[\Psi(n)+\Psi(n-m)-\Psi(2 n-m)-\Psi(1)], \\
& F_{2}(n)=\sum_{k=1}^{\infty} \frac{\Gamma^{2}(n+k)}{k ! \Gamma(2 n+k)} \frac{1}{k^{2}}, \quad F_{3}(n)=\sum_{m=1}^{n-1} \frac{\Gamma(n) \Gamma(n-m)}{\Gamma(2 n-m)} \frac{1}{m^{2}} \\
& F_{4}(n)=\sum_{m=1}^{n-1} \frac{\Gamma(n+m)}{m ! \Gamma(n)} \frac{1}{m^{2}}
\end{aligned}
$$

Our purpose here to show the calculatio of the series $F_{i}(n)$. The most important point is the following: if some series has the following form

$$
\tilde{F}(n)=\sum_{m=1}^{n-1} \tilde{f}(n, m),
$$

then it is important to reprsent it as

$$
\tilde{F}(n)=\sum_{m=1}^{n-1} \hat{f}(m),
$$

where the new function $\hat{f}(m)$ is $n$-independent.

Technically, the series $F_{i}(n)$ can be evaluated by considering a connection between $F_{i}(n+1)$ and $F_{i}(n)$, which can be expressed as a difference equation of the following type:

$$
\tilde{C}(n+1) \tilde{F}(n+1)-\tilde{C}(n) \tilde{F}(n)=\tilde{F}^{(1)}(n)
$$


with some coefficient functions $\tilde{C}(n)$. Usually the new function $\tilde{F}^{(1)}(n)$ can be summed by standard formulas from techbooks. If it is not the case, then it is necessary to repear the above procedure for the function $\tilde{F}^{(1)}(n)$, then we will come to new function, e.g., $\tilde{F}^{(2)}(n)$. So, repeating the above procedure for the functions $\tilde{F}^{(i)}(n)$, we will come to the one $\tilde{F}^{(i+1)}(n)$, which can be summed by standard formulas from techbooks.

For all the above functions $F_{i}(n)(i=1,2,3,4)$ we should repeat the procedure twicely. Is it not so convenient. More simple to consider the new functions $F_{i}(n, a)$ :

$$
\begin{aligned}
& F_{1}(n, a)=\sum_{m=1}^{n-1} \frac{\Gamma(n+a) \Gamma(n-m+a)}{m \Gamma(2 n-m+a) \Gamma(1+a)}, \quad F_{2}(n, a)=\sum_{k=1}^{\infty} \frac{\Gamma^{2}(n+k)}{k ! \Gamma(2 n+k)} \frac{1}{k-a}, \\
& F_{3}(n, a)=\sum_{m=1}^{n-1} \frac{\Gamma(n) \Gamma(n-m)}{\Gamma(2 n-m)} \frac{1}{m-a}, \quad F_{4}(n, a)=\sum_{m=1}^{n-1} \frac{\Gamma(n+m)}{m ! \Gamma(n)} \frac{1}{m-a},
\end{aligned}
$$

which can be used to reproduce $F_{i}(n)$ as

$$
F_{i}(n)=\left.\frac{d}{d a} F_{i}(n, a)\right|_{a=0} .
$$

The new functions $F_{i}(n, a)$ can be evaluated with the usage of the above procedure only one time. We will have

$$
\tilde{C}_{i}(n+1, a) \tilde{F}_{i}(n+1 . a)-\tilde{C}_{i}(n, a) \tilde{F}_{i}(n, a)=\tilde{F}_{i}^{(1)}(n, a),
$$

where the new functions $\bar{F}_{i}^{(1)}(n)$ can be summed by standard formulas from techbooks.

Here we will present the exact evaluation only the function $F_{1}(n, a)$. The other functions can be evaluated similiry and we will show only the basic steps.

6.1. $F_{1}(n, a)$

Consider $F_{1}(n+1, a)$ and express it through $F_{1}(n, a)$ as

$$
\begin{aligned}
& F_{1}(n+1, a)=\sum_{m=1}^{n} \frac{\Gamma(n+a+1) \Gamma(n-m+a+1)}{m \Gamma(2 n-m+a+2) \Gamma(1+a)}=\frac{\Gamma(n+a+1)}{n \Gamma(n+a+2) \Gamma(1+a)} \\
& +\sum_{m=1}^{n-1} \frac{\Gamma(n+a) \Gamma(n-m+a)}{m \Gamma(2 n-m+a) \Gamma(1+a)} \frac{(n+a)(n-m+a)}{m(2 n-m+a)(2 n+1-m+a)} .
\end{aligned}
$$

Expanding the last factor, we have after some little algebra

$$
\begin{aligned}
& \frac{(n-m+a)}{m(2 n-m+a)(2 n+1-m+a)}=\frac{(n+a)}{(2 n+a)(2 n+1+a)}\left[\frac{1}{m}+\frac{1}{2 n-m+a}\right] \\
& -\frac{n+1}{2 n+1+a} \frac{1}{(2 n-m+a)(2 n+1-m+a)},
\end{aligned}
$$

that leads for the r.h.s. of (152)

$$
\begin{aligned}
& \frac{1}{n(n+1+a)}+\frac{(n+a)^{2}}{(2 n+a)(2 n+1+a)}\left[F_{1}(n, a)+\sum_{m=1}^{n-1} \frac{\Gamma(n+1+a) \Gamma(n-m+a)}{\Gamma(2 n+1-m+a) \Gamma(1+a)}\right] \\
& -\frac{(n+1)(n+a)}{2 n+1+a} \sum_{m=1}^{n-1} \frac{\Gamma(n+1+a) \Gamma(n-m+a)}{\Gamma(2 n+2-m+a) \Gamma(1+a)} .
\end{aligned}
$$


The last two series can be evaluated since

$$
\begin{aligned}
& \sum_{m=1}^{n-1} \frac{\Gamma(n+a) \Gamma(n-m+a)}{\Gamma(2 n+\alpha-m+a) \Gamma(1+a)}=\sum_{m=1}^{n-1} \frac{\Gamma(n+a) \Gamma(m+a)}{\Gamma(n+m+\alpha+a) \Gamma(1+a)} \\
& =\frac{\Gamma(n+a)}{\Gamma(1+a)} \hat{\Phi}_{1}(n-2, a+1, n+\alpha+a+1)
\end{aligned}
$$

where the function $\hat{\Phi}_{1}(n, a, b)$ is defined and evaluated in Appendix A. Using these calculations

$$
\hat{\Phi}_{1}(n-2, a+1, n+\alpha+a+1)=\frac{1}{n+\alpha-1}\left[\frac{\Gamma(1+a)}{\Gamma(n+\alpha+a)}-\frac{\Gamma(n+a)}{\Gamma(2 n+\alpha+a-1)}\right],
$$

we have for the above series

$$
\sum_{m=1}^{n-1} \frac{\Gamma(n+a) \Gamma(n-m+a)}{\Gamma(2 n+\alpha-m+a) \Gamma(1+a)}=\frac{1}{n+\alpha-1}\left[\frac{\Gamma(1+a)}{\Gamma(n+\alpha+a)}-\frac{\Gamma(n+a)}{\Gamma(2 n+\alpha+a-1)}\right] \frac{\Gamma(n+a)}{\Gamma(1+a)} .
$$

Putting the result to (153), we have

$$
\begin{aligned}
& \frac{1}{n(n+1+a)}+\frac{(n+a)^{2}}{(2 n+a)(2 n+1+a)}\left[F_{1}(n, a)\right. \\
& \left.+\frac{1}{n}\left(\frac{\Gamma(n+a)}{\Gamma(n+1+a)}-\frac{\Gamma^{2}(n+a)}{\Gamma(2 n+a) \Gamma(1+a)}\right)\right] \\
& -\frac{(n+a)}{2 n+1+a}\left(\frac{\Gamma(n+a)}{\Gamma(n+12+a)}-\frac{\Gamma^{2}(n+a)}{\Gamma(2 n+1+a) \Gamma(1+a)}\right) .
\end{aligned}
$$

After some algebra, we have for $F_{1}(n+1, a)$ :

$$
\begin{aligned}
F_{1}(n+1, a)= & \frac{(n+a)^{2}}{(2 n+a)(2 n+1+a)} F_{1}(n, a)+\frac{3 n-2 a}{n(2 n+a)(2 n+1+a)} \\
& +\frac{a}{n} \frac{\Gamma(n+a) \Gamma(n+a+1)}{\Gamma(2 n+2+a) \Gamma(1+a)} .
\end{aligned}
$$

Consider

$$
F_{1}(n, a)=C_{1}(n, a) \bar{F}_{1}(n, a)
$$

with

$$
C_{1}(n, a)=\frac{\Gamma^{2}(n+a)}{\Gamma(2 n+a) \Gamma(1+a)}
$$

Then, we have

$$
\begin{aligned}
\bar{F}_{1}(n+1, a) & =\bar{F}_{1}(n, a)+\frac{3 n-2 a}{n} \frac{\Gamma(2 n+a) \Gamma(1+a)}{\Gamma^{2}(n+1+a)}+\frac{a}{n(n+a)} \\
& =\bar{F}_{1}(1, a)+\sum_{m=1}^{n}\left(\frac{3 n-2 a}{n} \frac{\Gamma(2 m+a) \Gamma(1+a)}{\Gamma^{2}(m+1+a)}+\frac{a}{m(m+a)}\right) .
\end{aligned}
$$

Since $F_{1}(1, a)=0$, then $\bar{F}_{1}(1, a)=0$. Thus, we obtain the final result for $F_{1}(n, a)$ :

$$
F_{1}(n, a)=\frac{\Gamma^{2}(n+a)}{\Gamma(2 n+a) \Gamma(1+a)} \sum_{m=1}^{n-1}\left(\frac{3 n-2 a}{n} \frac{\Gamma(2 m+a) \Gamma(1+a)}{\Gamma^{2}(m+1+a)}+\frac{a}{m(m+a)}\right) .
$$


Then,

$$
\begin{aligned}
F_{1}(n, 0)= & 3 \frac{\Gamma^{2}(n)}{\Gamma(2 n)} \sum_{m=1}^{n-1} \frac{\Gamma(2 m)}{\Gamma^{2}(m+1)}=3 \frac{(n !)^{2}}{(2 n) !} W_{1} \\
F_{1}(n)= & \frac{\Gamma^{2}(n)}{\Gamma(2 n)}\left[S_{2}(2 n-1)+\sum_{m=1}^{n-1} \frac{\Gamma(2 m)}{\Gamma^{2}(m+1)}\right. \\
& \left.\times\left[3\left\{2 S_{2}(m-1)-S_{2}(2 m-1)-2 S_{2}(n-1)+S_{2}(2 n-1)\right\}+\frac{4}{m}\right]\right] \\
= & \frac{\Gamma^{2}(n)}{\Gamma(2 n)}\left[\bar{S}_{2}+3\left(\bar{S}_{1} W_{1}-\bar{W}_{1,1}\right)-6\left(S_{1} W_{1}-W_{1,1}\right)+4 W_{2}\right]
\end{aligned}
$$

where $S_{ \pm a, \ldots} \equiv S_{ \pm a, \ldots}(n-1), \bar{S}_{ \pm a, \ldots} \equiv S_{ \pm a, \ldots}(2 n-1)$ (see definition of $S_{ \pm a, \ldots}(n)$ in $(42), W_{ \pm a, \ldots} \equiv$ $W_{ \pm a, \ldots}(n-1)$ and $\bar{W}_{ \pm a, \ldots} \equiv W_{ \pm a, \ldots}(2 n-1)$ with

$$
W_{a}(n)=\sum_{m=1}^{n} \frac{\hat{C}_{m}^{-1}}{m^{a}}, W_{a, b, c, \cdots}(n)=\sum_{m=1}^{n} \frac{\hat{C}_{m}^{-1}}{m^{a}} S_{b, c, \cdots}(m)
$$

6.2. $F_{i}(n, a)(i=2,3,4)$

Consider $F_{i}(n+1, a) i=2,3,4$ and express it through $F_{i}(n, a)$ as in the previous subsection

$$
F_{i}(n+1, a)=K_{i}(n, a) F_{i}(n, a)+f_{i}(n, a),
$$

where

$$
K_{2}(n, a)=\frac{(n+a)^{2}}{(2 n+a)(2 n+1+a)}, \quad K_{3}(n, a)=\frac{n(n-a)}{(2 n-a)(2 n+1-a)}, \quad K_{4}(n, a)=\frac{n+a}{n}
$$

and

$$
\begin{aligned}
& f_{2}(n, a)=\frac{3 n-2 a}{n(2 n+a)(2 n+1+a)}-\frac{n[n(4 n+3)+a(3 n+2)]}{(2 n+a)(2 n+a+1)} \frac{\Gamma^{2}(n)}{\Gamma(2 n+2)}, \\
& f_{3}(n, a)=\frac{3 n^{2}-3 a n+a^{2}}{n(2 n-a)(2 n+1-a)}+\frac{a}{2(2 n-a)(2 n-a+1)} \frac{\Gamma^{2}(n)}{\Gamma(2 n)} \\
& f_{4}(n, a)=\frac{3 n-2 a}{n-a} \frac{\Gamma(2 n)}{\Gamma^{2}(n+1)}-\frac{1}{n} .
\end{aligned}
$$

Consider

$$
F_{i}(n, a)=C_{i}(n, a) \bar{F}_{i}(n, a), f_{i}(n, a)=C_{i}(n, a) \bar{f}_{i}(n, a)
$$

with

$$
C_{2}(n, a)=\Gamma(1+a) C_{2}(n, a), \quad C_{3}(n, a)=\frac{\Gamma(n) \Gamma(n-a)}{\Gamma(2 n-a)}, \quad C_{4}(n, a)=\frac{\Gamma(n-a)}{\Gamma(n)} .
$$

Then, we have

$$
\bar{F}_{i}(n+1, a)=\bar{F}_{i}(n, a)+\bar{f}_{i}(n, a)=\bar{F}_{i}(1, a)+\sum_{m=1}^{n} \bar{f}_{i}(m, a),
$$


where

$$
\begin{aligned}
& \bar{f}_{2}(n, a)=\frac{3 n+2 a}{n} \frac{\Gamma(2 n+a)}{\Gamma^{2}(n+1+a)}-\left(4 n+3+\frac{a}{n}(3 n+2)\right) \frac{\Gamma^{2}(n+1)}{\Gamma(2 n+2)} \frac{\Gamma(2 n+a)}{\Gamma^{2}(n+1+a)}, \\
& \bar{f}_{3}(n, a)=\frac{3 n^{2}-3 a n+a^{2}}{n(n-a)} \frac{\Gamma(2 n-a)}{\Gamma(n+1-a) \Gamma(n+1)}+\frac{a}{2 n} \frac{\Gamma(n)}{\Gamma(2 n)} \frac{\Gamma(2 n+a)}{\Gamma(n+1+a)}, \\
& \bar{f}_{4}(n, a)=\frac{3 n-a}{n-a} \frac{\Gamma(2 n)}{\Gamma(n+1-a) \Gamma(n+1)}-\frac{\Gamma(n)}{\Gamma(n+1-a)} .
\end{aligned}
$$

Since $F_{j}(1, a)=0(j=3,4)$ and $F_{2}(1, a)=(\Psi(1+a)-\Psi(1)-1) /(a-1)$, then

$$
\bar{F}_{j}(1, a)=0(j=3,4), \bar{F}_{2}(1, a)=\frac{1}{\Gamma(1-a)}(1-\Psi(1+a)+\Psi(1)) .
$$

Thus, we obtain the final result for $F_{i}(n, a)$ :

$$
F_{i}(n, a)=C_{i}(n, a)\left[\bar{F}_{j}(1, a)+\sum_{m=1}^{n-1} \bar{f}_{i}(m, a)\right] .
$$

Then,

$$
F_{2}(n, 0)=\frac{\Gamma^{2}(n)}{\Gamma(2 n)}\left[\bar{S}_{1}-2 S_{1}+3 W_{1}\right], \quad F_{3}(n, 0)=3 \frac{(n !)^{2}}{(2 n) !} W_{1}, \quad F_{4}(n, 0)=\frac{\Gamma^{2}(n)}{\Gamma(2 n)}\left[3 W_{1}-S_{1}\right]
$$

and

$$
\begin{aligned}
F_{2}(n)= & \frac{\Gamma^{2}(n)}{\Gamma(2 n)}\left[\zeta_{2}-\frac{1}{2}\left(\bar{S}_{2}-2 S_{2}+\left(\bar{S}_{1}-2 S_{1}\right)^{2}\right)-3\left(\bar{S}_{1} W_{1}-\bar{W}_{1,1}\right)\right. \\
& \left.+6\left(S_{1} W_{1}-W_{1,1}\right)-4 W_{2}\right], \\
F_{3}(n)= & \frac{\Gamma^{2}(n)}{\Gamma(2 n)}\left[\frac{1}{2} \bar{S}_{2}+3\left(\bar{S}_{1} W_{1}-\bar{W}_{1,1}\right)-3\left(S_{1} W_{1}-W_{1,1}\right)+3 W_{2}\right], \\
F_{4}(n)= & \frac{\Gamma^{2}(n)}{\Gamma(2 n)}\left[3\left(S_{1} W_{1}-W_{1,1}\right)-W_{2}-\frac{1}{2}\left(S_{1}^{2}-S_{2}\right)\right] .
\end{aligned}
$$

Taking the results for $F_{i}(n)(i=1,2,3,4)$ together, we have for $P_{126}$ :

$$
\begin{aligned}
P_{125}= & -\frac{\hat{N}}{\left(q^{2}\right)^{2}} \sum_{n=1} \frac{(-x)^{n}}{2 n} \frac{(n !)^{2}}{(2 n) !}\left\{\frac{1}{\varepsilon^{2}}-\frac{1}{\varepsilon}\left(S_{1}+\ln x\right)+4 \bar{S}_{1} S_{1}-\frac{3}{2}\left(5 S_{1}^{2}+S_{2}\right)-\zeta_{2}\right. \\
& \left.+\frac{2}{n} S_{1}-S_{1} \ln x+\frac{1}{2} \ln ^{2} x\right\},
\end{aligned}
$$

where all series $W_{ \pm a, \ldots}$ and $\bar{W}_{ \pm a, \ldots}$ are canceled.

\subsection{Properties of Series}

This scheme was successfully used to calculate the two-loop two-point $[28,29,132,133,136,141]$ and three-point diagrams $[56,142,143]$ with one nonzero mass. This procedure is very powerful, but rather complicated. However, there are some simplifications based on representations of series of Feynman integrals.

Indeed, the inverse-mass expansion of two-loop two-point and three-point diagrams (the diagrams are complicated two-loop Feynman integrals that do not have cuts of three massive particles. 
thus, their results should be expressed as combinations of Polylogarithms. Note that we consider only three-point diagrams with independent upward momenta $q_{1}$ and $q_{2}$, which satisfy the conditions $q_{1}^{2}=q_{2}^{2}=0$ and $\left(q_{1}+q_{2}\right)^{2} \equiv q^{2} \neq 0$, where $q$ is downward momentum) with one nonzero mass (massless and massive propagators are shown by dashed and solid lines, respectively), can be considered as

$$
\begin{aligned}
\mathrm{FI}= & \frac{\hat{N}}{q^{2 \alpha}} \sum_{n=1} C_{n}(\eta x)^{n}\left\{F_{0}(n)+\left[\ln x F_{1,1}(n)+\frac{1}{\varepsilon} F_{1,2}(n)\right]\right. \\
& \left.+\left[\ln ^{2} x F_{2,1}(n)+\frac{1}{\varepsilon} \ln x F_{2,2}(n)+\frac{1}{\varepsilon^{2}} F_{2,3}(n)+\zeta(2) F_{2,4}(n)\right]+\cdots\right\},
\end{aligned}
$$

where $x=q^{2} / m^{2}$ (as it was shown in (149), $\eta=1$ or -1 and $\alpha=1$ and 2 for two-point and three-point cases, respectively. The normalization $\hat{N}$ is defined below Equation (149). Moreover,

$$
C_{n}=\frac{(n !)^{2}}{(2 n) !} \equiv \hat{C}_{n}
$$

for diagrams with two-massive-particle-cuts ( $2 m$-cuts). For the diagrams with one-massive-particlecuts (m-cuts) $C_{n}=1$.

For $m$-cut case, the coefficients $F_{N, k}(n)$ should have the form

$$
F_{N, k}(n) \sim \frac{S_{ \pm a, \ldots}}{n^{b}}, \frac{\zeta( \pm a)}{n^{b}}
$$

where $S_{ \pm a, \ldots} \equiv S_{ \pm a, \ldots}(j-1)$ are harmonic sums defined in (42) and $\zeta( \pm a)$ are the Euler-Zagier constants

$$
\zeta( \pm a)=\sum_{m=1}^{\infty} \frac{( \pm 1)^{m}}{m^{a}}, \zeta( \pm a, \pm b, \pm c, \cdots)=\sum_{m=1}^{\infty} \frac{( \pm 1)^{m}}{m^{a}} S_{ \pm b, \pm c, \cdots}(m-1)
$$

For $2 m$-cut case, the coefficients $F_{N, k}(n)$ can be more complicated

$$
F_{N, k}(n) \sim \frac{S_{ \pm a, \ldots}}{n^{b}}, \frac{V_{a, \ldots}}{n^{b}}, \frac{W_{a, \ldots}}{n^{b}},
$$

where $S_{ \pm a, \ldots} \equiv S_{ \pm a, \ldots}(n-1)$ and $W_{ \pm a, \ldots}$ are defined in Equations (42) and (162), respectively, and $V_{ \pm a, \ldots} \equiv V_{ \pm a, \ldots}(j-1)$ with

$$
V_{a}(j)=\sum_{m=1}^{j} \frac{\hat{C}_{m}}{m^{a}}, \quad V_{a, b, c, \cdots}(j)=\sum_{m=1}^{j} \frac{\hat{C}_{m}}{m^{a}} S_{b, c, \cdots}(m),
$$

The terms $\sim V_{a, \ldots .}$ and $\sim W_{a, \ldots .}$ can come only in the $2 m$-cut case. The origin of the appearance of these terms is the product of series (174) with the different coefficients $C_{n}=1$ and $C_{n}=\hat{C}_{n}$.

\subsection{Other Examples}

As an example, consider two-loop two-point diagrams $I_{1}$ and $I_{12}$ studied in [56].

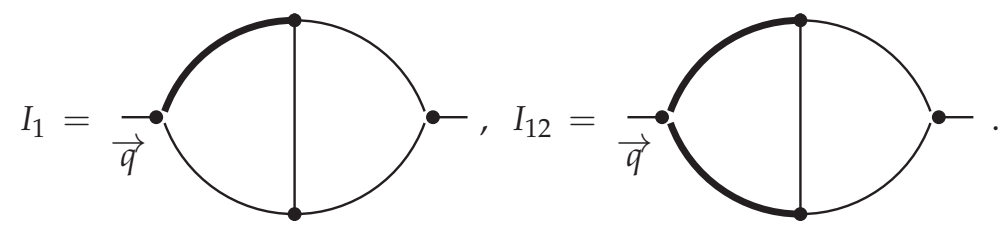


Their results are

$$
\begin{aligned}
I_{1} & =-\frac{\hat{N}}{q^{2}} \sum_{n=1} \frac{(-x)^{n}}{n}\left\{\frac{1}{2} \ln ^{2} x-\frac{2}{n} \ln x+\zeta(2)+2 S_{2}-2 \frac{S_{1}}{n}+\frac{3}{n^{2}}\right\}, \\
I_{12} & =-\frac{\hat{N}}{q^{2}} \sum_{n=1} \frac{(-x)^{n}}{n^{2}}\left\{\frac{1}{n}+\frac{(n !)^{2}}{(2 n) !}\left(-2 \ln x-3 W_{1}+\frac{2}{n}\right)\right\} .
\end{aligned}
$$

From (181) one can see that the corresponding functions $F_{N, k}(n)$ have the form

$$
F_{N, k}(n) \sim \frac{1}{n^{3-N}}, \quad(N \geq 2),
$$

if we introduce the following complexity of the sums $(\bar{\Phi}=(S, V, W))$

$$
\bar{\Phi}_{ \pm a} \sim \bar{\Phi}_{ \pm a_{1}, \pm a_{2}} \sim \bar{\Phi}_{ \pm a_{1}, \pm a_{2}, \cdots, \pm a_{m}} \sim \zeta_{a} \sim \frac{1}{n^{a}}, \quad\left(\sum_{i=1}^{m} a_{i}=a\right) .
$$

The number $3-N$ determines the level of transcendentality (or complexity, or weight) of the coefficients $F_{N, k}(n)$. The property greatly reduces the number of the possible elements in $F_{N, k}(n)$. The level of transcendentality decreases if we consider the singular parts of diagrams and/or coefficients in front of $\zeta$-functions and of logarithm powers. Thus, finding the parts we can predict the rest using the ansatz based on the results already obtained, but containing elements with a higher level of transcendentality.

Other two-loop two-point integrals in [56] have similar form. They were exactly calculated by differential equation method $[28,136]$.

Now we consider two-loop three-point diagrams, $P_{5}$ and $P_{12}$ :

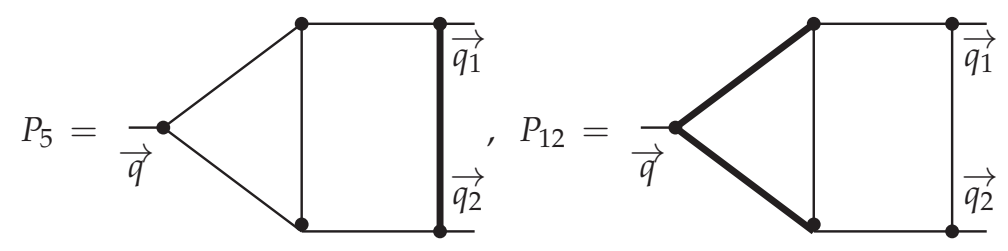

Their results are (see [56]):

$$
\begin{aligned}
P_{5}= & \frac{\hat{N}}{\left(q^{2}\right)^{2}} \sum_{n=1} \frac{x^{n}}{n}\left\{-6 \zeta_{3}+2 S_{1} \zeta_{2}+6 S_{3}-2 S_{1} S_{2}+4 \frac{S_{2}}{n}-\frac{S_{1}^{2}}{n}+2 \frac{S_{1}}{n^{2}}\right. \\
& \left.+\left(-4 S_{2}+S_{1}^{2}-2 \frac{S_{1}}{n}\right) \ln x+S_{1} \ln ^{2} x\right\} \\
P_{12}= & \frac{\hat{N}}{\left(q^{2}\right)^{2}} \sum_{n=1} \frac{(-x)^{n}}{n^{2}} \frac{(n !)^{2}}{(2 n) !}\left\{\frac{2}{\varepsilon^{2}}+\frac{2}{\varepsilon}\left(S_{1}-3 W_{1}+\frac{1}{n}-\ln x\right)-6 W_{2}-18 W_{1,1}\right. \\
& \left.-13 S_{2}+S_{1}^{2}-6 S_{1} W_{1}+2 \frac{S_{1}}{n}+\frac{2}{n^{2}}-2\left(S_{1}+\frac{1}{n}\right) \ln x+\ln ^{2} x\right\} .
\end{aligned}
$$

Now the coefficients $F_{N, k}(n)$ have the form

$$
F_{N, k}(n) \sim \frac{1}{n^{4-N}}, \quad(N \geq 3),
$$

The diagram $P_{5}$ (and also $P_{1}, P_{3}$ and $P_{6}$ in [56]) was calculated exactly by differential equation method $[28,29,136-140]$ To find the results for $P_{12}$ (and also all others in [56]) we have used the knowledge of the several $n$ terms in the inverse-mass expansion (174) (usually less than $n=100$ ) and the following arguments: 
- If a two-loop two-point diagram with a "similar topology" (for example, $I_{12}$ for $P_{12}$, etc.) was already calculated, we should consider a similar set of basic elements for corresponding $F_{N, k}(n)$ of two-loop three-point diagrams but with a higher level of complexity.

- Let the diagram under consideration contain singularities and/or powers of logarithms. Since the coefficients are very simple before the leading singularity, or the largest degree of the logarithm, or the largest $\zeta$-function, they can often be predicted directly from the first few terms of the expansion.

Moreover, often we can calculate the singular part using a different technique (see [56] for extraction of $\sim W_{1}(n)$ part). Then we should expand the singular parts, find the main elements and try to use them (with the corresponding increase in the level of complexity) in order to predict the regular part of the diagram. If we need to find $\varepsilon$-suppressed terms, we should increase the level of complexity of the corresponding basic elements.

Later, using the ansatz for $F_{N, k}(n)$ and several terms (usually less than 100) in the above expression, which can be exactly calculated, we obtain a system of algebraic equations for the parameters of the ansatz. Solving the system, we can obtain the analytical results for Feynman integrals without exact calculations. To check the results, we only need to calculate a few more terms in the above inverse-mass expansion (174) and compare them with the predictions of our anzatz with the fixed coefficients indicated above.

Thus, the considered arguments give a possibility to find results for many complicated two-loop three-point diagrams without direct calculations. Several process options have been successfully used to calculate Feynman diagrams for many processes (see [56,132,133,142,144-147]).

Note that properties similar to (183) and (186) were recently observed [66-69] in the so-called double operator-product-expansion limit of some four-point diagrams.

\subsection{Modern Technique of Massive Diagrams}

Coefficients have the structure (183) and (186) with the rule (184). Note that these conditions greatly reduce the number of possible harmonic sums. In turn, the restriction is associated with a specific form of differential equations for the Feynman integrals under consideration. Differentials equations can be formally represented as [108-112]

$$
\left((x+a) \frac{d}{d x}-\bar{k} \varepsilon\right) \text { FI }=\text { less complicated diagrams }\left(\equiv \mathrm{FI}_{1}\right),
$$

with some number $a$ and some function $\bar{k}(x)$. This form is generated by IBP procedure for diagrams including an inner $n$-leg one-loop subgraph, which in turn contains the product $k^{\mu_{1}} \ldots k^{\mu_{m}}$ of its internal momenta $k$ with $m=n-3$.

Indeed, for ordinary degrees $\alpha_{i}=1+a_{i} \varepsilon$ with arbitrary $a_{i}$ of subgraph propagators, the IBP relation (12) gives the coefficient $d-2 \alpha_{1}-\sum_{i=2}^{p} \alpha_{i}+m \sim \varepsilon$ for $m=n-3$. Important examples of applying the rule are the diagrams $I_{1}, I_{12}$ and $P_{5}, P_{12}, P_{126}$ (for the case $n=2$ and $n=3$ ) and also the diagrams in in Ref. [148] (for the case $n=3$ and $N=4$ ). However, we note that the results for the non-planar diagrams (see Figure 3 of [56]) obey the Equation (186) but their subgraphs do not comply with the above rule. The disagreements may be related to the on-shall vertex of the subgraph, but this requires additional research.

Taking the set of less complicated Feynman integrals $\mathrm{FI}_{1}$ as diagrams having internal $n$-leg subgraphs, we get their result stucture similar to the one given above (186), but with a lower level of complexity.

So, the integrals $\mathrm{FI}_{1}$ should obey to the following equation

$$
\left(\left(x+a_{1}\right) \frac{d}{d x}-\bar{k}_{1} \varepsilon\right) \mathrm{FI}_{1}=\text { less }^{2} \operatorname{complicated} \operatorname{diagrams}\left(\equiv \mathrm{FI}_{2}\right) .
$$


Thus, we will have the set of equations for all Feynman integrals $\mathrm{FI}_{\mathrm{n}}$ as

$$
\left(\left(x+a_{n}\right) \frac{d}{d x}-\bar{k}_{n} \varepsilon\right) \mathrm{FI}_{n}=\text { less }^{n+1} \text { complicated diagrams }\left(\equiv \mathrm{FI}_{\mathrm{n}+1}\right),
$$

with the last integral $\mathrm{FI}_{\mathrm{n}+1}$ contains only tadpoles.

Moreover, following [149,150], we can reconstruct the above set of inhomogeneous equations as the the homogeneous matrix equation (for complicated diagrams, there is an extension in Ref. [151]) (see Ref. [152-154] containg methods to obtain the equation)

$$
\frac{d}{d x} \widehat{F I}-\varepsilon \widehat{K} \widehat{F I}=0
$$

for the vector

$$
\widehat{F I}=\left(\begin{array}{l}
\mathrm{FI} \\
\mathrm{FI}_{1} /{ }^{\prime \prime} \\
\cdots \\
\mathrm{FI}_{\mathrm{n}} /{ }^{\prime \prime \mathrm{n}}
\end{array}\right),
$$

where the matrix $\widehat{K}$ contains the functions $\bar{k}_{j} /\left(x+a_{j}\right)$ as its elements. The form (190) is called as the "canonic basic". It is now very popular (see, for example, recent papers in Refs. [155-163]).

Please note that for real calculations of $\mathrm{FI}_{n}$ it is convenient to replace

$$
\mathrm{FI}_{\mathrm{n}}=\widetilde{\mathrm{FI}}_{\mathrm{n}} \overline{\mathrm{FI}}_{\mathrm{n}}
$$

where the term $\overline{\mathrm{FI}}_{n}$ obeys the corresponding homogeneous equation

$$
\left(\left(x+a_{n}\right) \frac{d}{d x}-\bar{k}_{n} \varepsilon\right) \overline{\mathrm{FI}}_{n}=0 .
$$

The replacement simplifies the above equation (189) to the following form

$$
\left(x+a_{n}\right) \frac{d}{d x} \widetilde{\mathrm{FI}}_{n}=\widetilde{\mathrm{FI}}_{n+1} \frac{\overline{\mathrm{FI}}_{n+1}}{\overline{\mathrm{FI}}_{n}},
$$

having the solution

$$
\widetilde{\mathrm{FI}}_{n}(x)=\int_{0}^{x} \frac{d x_{1}}{x_{1}+a_{n}} \widetilde{\mathrm{FI}}_{n+1}\left(x_{1}\right) \frac{\overline{\mathrm{FI}}_{n+1}\left(x_{1}\right)}{\overline{\mathrm{FI}}_{n}\left(x_{1}\right)}
$$

Usually there are some cancellations in the ratio $\overline{\mathrm{FI}}_{n+1} / \overline{\mathrm{FI}}_{n}$ and sometimes it is equal to 1 . In the last case, the equation (193) coincides wuth the definition of Goncharov Polylogariths (see [164] and the references therein).

The series (181), (182) and (185) can be expressed as a combination of the Nilson [165] and Remiddi-Vermaseren [166] polylogarithms with weight $4-N$ (see [56,142]). More complicated cases were examined in [167].

\section{7. $\mathcal{N}=4 \mathrm{SYM}$}

Note that both in the massless case and in the massive case the results of scalar diagrams have an important property: all elements have the form (see Equation (184))

$$
\frac{\Phi_{a_{1}, a_{2}, \ldots}\left(n+n_{0}\right)}{\left(n+n_{0}\right)^{b}} \text { or } \frac{\Phi_{a_{1}, a_{2}, \ldots}\left(n+n_{0}\right)}{\left(n+n_{0}+1\right)^{b}},
$$


where the quantity $\bar{a}+b\left(\bar{a}=\sum_{i=1}\left|a_{i}\right|\right)$ is fixed for each order of expansion in $\varepsilon$. For integrals $I_{1}(1, n)$ and $I_{2}(1, n)$ (and also $I_{1}(n+1, n)$ and $I_{2}(n+1, n)$ ), this property is even more strict, since in this case $b=0$.

Unfortunately, calculations in QCD (or another theory) mix orders of magnitude over $\varepsilon$. Moreover, in expansions of diagrams containing propagators with non-trivial numerators (for example, as propagators of quarks and gluons) this property is also violated.

It is an amazing fact that the property (194) in its most strict form $b=0$ is restored for diagonal elements of anomalous dimensions [47-50] and DIS coefficient functions [51] within $N=4$ SYM.

The anomalous dimensions of the twist-2 Wilson operators govern the Bjorken scaling violation for parton distributions (三 matrix elemens of the twist-2 Wilson operators) in the framework of Quantum Chromodynamics.

Balitsky-Fadin-Kuraev-Lipatov [37] and Dokshitzer-Gribov-Lipatov-Altarelli-Parisi (DGLAP) [168 172 ] equations resum, respectively, the most important contributions $\sim \alpha_{S} \ln \left(1 / x_{B}\right)$ and $\sim \alpha_{s} \ln \left(Q^{2} / \Lambda^{2}\right)$ in different kinematical regions of the Bjorken variable $x_{B}$ and the "mass" $Q^{2}$ of the virtual photon in the lepton-hadron DIS and, thus, they are the cornerstone in analyses of the experimental data from lepton-nucleon and nucleon-nucleon scattering. In the supersymmetric generalization of QCD the equations are simplified drastically [36]. In the $\mathcal{N}=4$ SYM the eigenvalues of the matrix of anomalous dimesion contain only one universal function with shifted arguments $[47,48,173]$.

The three-loop result (note, that in an accordance with Ref. $[43,44]$ our normalization of $\gamma(n)$ contains the extra factor $-1 / 2$ in comparison with the standard normalization (see $[47,48]$ ) and differs by sign in comparison with one from Ref. [52-54]) for the universal anomalous dimension $\gamma_{u n i}(j)$ for $\mathcal{N}=4$ SYM is [50]

$$
\gamma_{u n i}(n)=\hat{a} \gamma_{u n i}^{(0)}(n)+\hat{a}^{2} \gamma_{u n i}^{(1)}(n)+\hat{a}^{3} \gamma_{u n i}^{(2)}(n)+\ldots, \quad \hat{a}=\frac{\alpha N_{c}}{4 \pi},
$$

where

$$
\begin{aligned}
& \frac{1}{4} \gamma_{u n i}^{(0)}(j+2)=-S_{1}(n) \\
& \frac{1}{8} \gamma_{u n i}^{(1)}(j+2)=\left(S_{3}(n)+\bar{S}_{-3}(n)\right)-2 \bar{S}_{-2,1}(n)+2 S_{1}(n)\left(S_{2}(n)+\bar{S}_{-2}(n)\right) \\
& \frac{1}{32} \gamma_{u n i}^{(2)}(j+2)=2 \bar{S}_{-3}(n) S_{2}(n)-S_{5}(n)-2 \bar{S}_{-2}(n) S_{3}(n)-3 \bar{S}_{-5}(n)+24 \bar{S}_{-2,1,1,1}(n) \\
& \quad+6\left(\bar{S}_{-4,1}(n)+\bar{S}_{-3,2}(n)+\bar{S}_{-2,3}(n)\right)-12\left(\bar{S}_{-3,1,1}(n)+\bar{S}_{-2,1,2}(n)+\bar{S}_{-2,2,1}(n)\right) \\
& \quad-\left(S_{2}(n)+2 S_{1}^{2}(n)\right)\left(3 \bar{S}_{-3}(n)+S_{3}(n)-2 \bar{S}_{-2,1}(n)\right)-S_{1}(n)\left(8 \bar{S}_{-4}(n)+\bar{S}_{-2}^{2}(n)\right. \\
& \left.\quad+4 S_{2}(n) \bar{S}_{-2}(n)+2 S_{2}^{2}(n)+3 S_{4}(n)-12 \bar{S}_{-3,1}(n)-10 \bar{S}_{-2,2}(n)+16 \bar{S}_{-2,1,1}(n)\right)
\end{aligned}
$$

with $S_{ \pm a, \pm b, \pm c, \ldots}(n)$ (see Equation (42)) and

$$
\bar{S}_{-a, b, c, \cdots}(n)=(-1)^{n} S_{-a, b, c, \ldots}(n)+S_{-a, b, c, \ldots}(\infty)\left(1-(-1)^{n}\right) .
$$

The expression (199) is the analytical continuation (to real and complex $n$ ) [174,175] of the harmonic sums $S_{-a, b, c, \cdots}(n)$ (see discussions andout the analytical continuation and its applications in Appendix C).

The results for $\gamma_{u n i}^{(3)}(n)$ [176-179], $\gamma_{u n i}^{(4)}(n)$ [180], $\gamma_{u n i}^{(5)}(n)$ [181] and $\gamma_{u n i}^{(6)}(n)$ [182] are obtained from the long-range asymptotic Bethe Equations $[183,184]$ for twist-two operators and the additional contribution of the wrapping corrections. The similar calculations for the anomalous dimension in the twist-three case can be found in [185-187]. 
Similary to the Equations (183) and (186) let us to introduce the transcendentality level $i$ for the harmonic sums $S_{ \pm a}(n)$ and and Euler-Zagier constants $\zeta( \pm a)$ in the following way

$$
S_{ \pm a, \pm b, \pm c, \cdots}(n) \sim \zeta( \pm a, \pm b, \pm c, \cdots) \sim 1 / n^{i}, \quad(i=a+b+c+\cdots) .
$$

Then, the basic functions $\gamma_{u n i}^{(0)}(n), \gamma_{u n i}^{(1)}(n)$ and $\gamma_{u n i}^{(2)}(n)$ are assumed to be of the types $\sim 1 / n^{i}$ with the levels $i=1, i=3$ and $i=5$, respectively. A violation of this property may be obtained from contributions of the terms appearing at a given order from previous orders of the perturbation theory. Such contributions could be generated and/or removed using an appropriate finite renormalization and/or redefinition of the coupling constant. But these terms do not appear in the $\overline{\mathrm{DR}}$-scheme [188].

It is known, that at the first three orders of perturbation theory (with the SUSY relation for the QCD color factors $C_{F}=C_{A}=N_{c}$ ) the most complicated contributions (with $i=1,3$ and 5 , respectively) are the same as in QCD [52-54]. This property allows one to find the universal anomalous dimensions $\gamma_{u n i}^{(0)}(n), \gamma_{u n i}^{(1)}(n)$ and $\gamma_{u n i}^{(2)}(n)$ without knowing all elements of the anomalous dimension matrix [47,48], which was verified for $\gamma_{u n i}^{(1)}(j)$ by the exact calculations in [49].

Note that in $\mathcal{N}=4$ SYM some partial cases of anomalous dimension are also known for the large couplings from string calculations and AdS/QFT correspondence [189-191]. We would like to note that if the property of the maximal transcendentality exists at low coupling, then sometimes it appeares at large couplings (see, for example, the results for the cusp anomalous dimension at low $[192,193]$ and large $[194,195]$ couplings, both of which are based on the Beisert-Eden-Staudacher Equation [196]). However, this is not true for Pomeron intercept, which results lose the property of maximal transcendentality at large couplings (see [50,197-200]). The reason of the difference in the results for the cusp anomalous dimension and Pomeron intercept is currently not clear. More research is required.

\section{Conclusions}

In this review, we presented the results of the calculation of some massless and massive two-loop Feynman integrals. In the massless case, we considered scalar two-point diagrams with a traceless product in the numerator of one propagator and diagrams depending on two momenta $q$ and $p$ when $p^{2}=0$. In the massive case, we studied the $1 / \mathrm{m}^{2}$-expansion of Feynman integrals. The similarity of the structure of the expansion coefficients of massless and massive diagrams is shown.

Evolutions of the most complicated parts: $\Phi_{i}(n)(i=1,2)$ for massless diagrams and $F_{j}(n)$ $(j=1,2,3,4)$ for massive diagrams (see Sections 3.2.1, 3.3.1, 6.1 and 6.2, respectively), are shown in details.

The main calculation method for $\Phi_{i}(n)$ and $F_{j}(n)$ is to build recurrence relations (i.e., the relationships between $\Phi_{i}(n)$ and $\Phi_{i}(n-1)$ and also $F_{j}(n)$ and $\left.F_{j}(n-1)\right)$, which ingomogeneous parts contains only simpler amounts. The calculation of the series in the ingomogeneous parts allows us to get complete information about the recurrence relations and, as a result, solve them and get the desired values for the studied $\Phi_{i}(n)(i=1,2)$ and $F_{j}(n)(j=1,2,3,4)$.

Note that this approach is close to the method of differential equations for calculating massive diagrams. This is not surprising, since the differential equations for any function correspond to recurrence relations for the coefficients of its expansion.

For the massless and massive diagrams under consideration, the level of transcendentality (or complexity, or weight) remains unchanged in any order of $\varepsilon$ (see Sections 6.3 and 6.4). Moreover, it decreases in the presence of logarithms or $\zeta$-functions. It is called as transcendentality principle. Its application leads to the possibility to get the results for most of integrals without direct calculations.

This property is violated in physical models, such as QCD, where propagators (both quarks and gluons) contain momenta in their numerators, which are responsible for mixing levels of complexity. However, this property is preserved (see Section 7) for diagonalized quantities, such as diagonal 
anomalous dimensions and coefficient functions in $N=4 \mathrm{SYM}$, which is an amazing but poorly studied property.

Author thanks the Organizing Committee of Helmholtz International Summer School "Quantum Field Theory at the Limits: from Strong Fields to Heavy Quarks" for invitation. He is grateful to Andrei Pikelner for his help with the Axodraw2.

Funding: This research received no external funding.

Acknowledgments: A.V.K. thanks the Organizing Committee of Helmholtz International Summer School "Quantum Field Theory at the Limits: from Strong Fields to Heavy Quarks" for their invitation.

Conflicts of Interest: The author declares no conflict of interest.

\section{Appendix A. Some Useful Formulas}

A. Consider firstly the simple series

$$
\hat{\Phi}_{1}(n ; a, b)=\sum_{m=0}^{n} \frac{\Gamma(m+a)}{\Gamma(m+b)} .
$$

It is conveninet to rewrite it throught ${ }_{2} F_{1}$-hypergeometric functions with the argument 1 , which are exactly expressed as a product of $\Gamma$-functions

$$
{ }_{2} F_{1}(a, b ; c ; 1)=\frac{\Gamma(c) \Gamma(c-a-b)}{\Gamma(c-a) \Gamma(c-b)} \text { for } c-a-b>0 .
$$

Consider $\hat{\Phi}_{1}(a, b)$ as

$$
\hat{\Phi}_{1}(n ; a, b)=\sum_{m=0}^{\infty} \frac{\Gamma(m+a)}{\Gamma(m+b)}-\sum_{m=n+1}^{\infty} \frac{\Gamma(m+a)}{\Gamma(m+b)}=\sum_{m=0}^{\infty}\left(\frac{\Gamma(m+a)}{\Gamma(m+b)}-\frac{\Gamma(m+a+n+1)}{\Gamma(m+b+n+1)}\right) .
$$

Using (A2), we have

$$
\hat{\Phi}_{1}(n ; a, b)=\frac{1}{b-a-1}\left[\frac{\Gamma(a)}{\Gamma(b-1)}-\frac{\Gamma(a+n+1)}{\Gamma(b+n)}\right] .
$$

Now we consider another series

$$
\hat{\Phi}_{2}(n ; \alpha, \beta)=\sum_{m=0}^{n} \frac{\Gamma(m+\beta+1)}{\Gamma(n-m+1+\alpha)} .
$$

Rewriting

$$
\frac{\Gamma(n-m+1+\alpha)}{\Gamma(1+\alpha)}=(-1)^{n-m} \frac{\Gamma(-\alpha)}{\Gamma(-\alpha-n+m)},
$$

we see that

$$
\begin{aligned}
\hat{\Phi}_{2}(n ; \alpha, \beta) & =\frac{(-1)^{n}}{\Gamma(-\alpha) \Gamma(1+\alpha)} \hat{\Phi}_{1}(-n-\alpha, \beta) \\
& =\frac{(-1)^{n}}{\Gamma(-\alpha) \Gamma(1+\alpha)} \frac{1}{\alpha+\beta+n}\left[\frac{\Gamma(-n-\alpha)}{\Gamma(\beta)}-\frac{\Gamma(1-\alpha)}{\Gamma(\beta+n+1)}\right] .
\end{aligned}
$$

Since

$$
\Gamma(-n-\alpha)=(-1)^{n} \frac{\Gamma(-\alpha) \Gamma(1+\alpha)}{\Gamma(n+1+\alpha)},
$$


we obtain the useful relation

$$
\hat{\Phi}_{2}(n ; \alpha, \beta)=\frac{1}{\alpha+\beta+n}\left[\frac{1}{\Gamma(n+1+\alpha) \Gamma(\beta)}+\frac{(-1)^{n}}{\Gamma(\alpha) \Gamma(\beta+n+1)}\right],
$$

which has important particular cases

$$
\hat{\Phi}_{2}(n ; \alpha=0, \beta)=\frac{1}{\beta+n} \frac{1}{\Gamma(n+1) \Gamma(\beta)}, \quad \hat{\Phi}_{2}(n ; \alpha, \beta=0)=\frac{(-1)^{n}}{\alpha+n} \frac{1}{\Gamma(n+1) \Gamma(\alpha)} .
$$

B. Consider $\bar{C}_{2}^{(4)}(0, n)$ considered in Equation (84). It has the form

$$
\begin{aligned}
\bar{C}_{2}^{(4)}(0, n) & =-\sum_{k=0}^{n+1} \frac{(-1)^{k} n ! S_{1}(k) S_{1}(n+1-k)}{k !(n-k+1) !} \\
& =-\left.\frac{\partial}{\partial x} \frac{\partial}{\partial y} \sum_{k=0}^{n+1} \frac{(-1)^{k} n ! \Gamma(1-x) \Gamma(1-y)}{\Gamma(k+1-x) \Gamma(n-k+2-y)}\right|_{x=0, y=0} .
\end{aligned}
$$

Taking the results (A9) we have

$$
\begin{aligned}
& \bar{C}_{2}^{(4)}(0, n)=-\left.\frac{\partial}{\partial x} \frac{\partial}{\partial x} \frac{n !}{n+2-x-y}\left[\frac{(-x) \Gamma(1-y)}{\Gamma(n+3-y)}+\frac{(-1)^{n} \Gamma(1-x)}{\Gamma(n+2-x)}\right]\right|_{x=0, y=0} \\
& =-\left.n ! \frac{\partial}{\partial x}\left[\frac{1}{(n+2-x)^{2}}\left(\frac{(-x)}{\Gamma(n+3)}+\frac{(-1)^{n} \Gamma(1-x)}{\Gamma(n+2-x)}\right)+\frac{(-x)}{(n+2-x)} \frac{S_{1}(n+2)}{\Gamma(n+3)}\right]\right|_{x=0} .
\end{aligned}
$$

Performing the derivative with respect of $x$, we can parametrize the final result in the form

$$
\bar{C}_{2}^{(4)}(0, n)=-\frac{n !}{n+2}\left[A_{1}(n)+(-1)^{n} A_{2}(n)\right],
$$

where

$$
\begin{aligned}
& A_{1}(n)=-\frac{1}{(n+2)^{2}} \frac{1}{\Gamma(n+2)}-\frac{S_{1}(n+2)}{\Gamma(n+3)}=-\frac{1}{\Gamma(n+3)}\left(S_{1}(n+2)+\frac{1}{n+2}\right) \\
& A_{2}(n)=\frac{2}{(n+2)^{2}} \frac{1}{\Gamma(n+2)}+\frac{S_{1}(n+1)}{\Gamma(n+3)}=\frac{1}{\Gamma(n+3)}\left(S_{1}(n+1)+\frac{2}{n+2}\right)=-A_{1}(n)(A 14
\end{aligned}
$$

So, we see that $\bar{C}_{2}^{(4)}(0, n)=0$ for even $n$ values.

C. Consider the series

$$
S_{1,1}(n)=\sum_{m=1}^{n} \frac{S_{1}(m)}{m}, S_{1,2}(n)=\sum_{m=1}^{n} \frac{S_{2}(m)}{m}, \sum_{m=1}^{n} \frac{S_{1}^{2}(m)}{m}
$$

and expess them trough standard sums $S_{i}(n)$ and the one $S_{2,1}(n)$.

For the first series $S_{1,1}(n)$ we have

$$
S_{1,1}(n)=\sum_{m=1}^{n} \frac{1}{m} \sum_{l=1}^{m} \frac{1}{l}=\sum_{l=1}^{m} \frac{1}{l} \sum_{m=l}^{n} \frac{1}{m}=\sum_{l=1}^{m} \frac{1}{l}\left(S_{1}(n)-S_{1}(l)+\frac{1}{l}\right) .
$$

Comparing 1.h.s. and r.h.s., we see

$$
S_{1,1}(n)=\frac{1}{2} \sum_{l=1}^{m} \frac{1}{l}\left(S_{1}(n)+\frac{1}{l}\right)=\frac{1}{2}\left(S_{1}^{2}(n)+S_{2}(n)\right) .
$$


By analogy with the case $S_{1,1}(n)$ we can obtain

$$
S_{1,2}(n)=\sum_{l=1}^{m} \frac{1}{l}\left(S_{1}(n)-S_{1}(l)+\frac{1}{l}\right)=S_{1}(n) S_{2}(n)+S_{2,1}(n)+S_{3}(n) .
$$

and

$$
\begin{aligned}
\sum_{m=1}^{n} \frac{S_{1}^{2}(m)}{m} & =\sum_{m=1}^{n} \frac{S_{1}(m)}{m} \sum_{l=1}^{m} \frac{1}{l}=\sum_{l=1}^{n} \frac{1}{l} \sum_{m=l}^{n} \frac{S_{1}(m)}{m} \\
& =\sum_{l=1}^{m} \frac{1}{l}\left[\sum_{m=1}^{n} \frac{S_{1}(m)}{m}-\sum_{m=1}^{l} \frac{S_{1}(m)}{m}+\frac{S_{1}(l)}{l}\right] .
\end{aligned}
$$

Taking the results (A17), we have

$$
\sum_{m=1}^{n} \frac{S_{1}^{2}(m)}{m}=\frac{1}{2}\left[\left(S_{1}^{2}(n)+S_{2}(n)\right) S_{1}(n)-\sum_{l=1}^{m} \frac{1}{l}\left(S_{1}^{2}(l)+S_{2}(l)\right)+2 S_{2,1}(n)\right] .
$$

Comparing 1.h.s. and r.h.s., we see

$$
\sum_{m=1}^{n} \frac{S_{1}^{2}(m)}{m}=\frac{1}{3}\left[\left(S_{1}^{2}(n)+S_{2}(n)\right) S_{1}(n)-S_{1,2}(n)+2 S_{2,1}(n)\right] .
$$

Taking the results (A18), we have the final result

$$
\sum_{m=1}^{n} \frac{S_{1}^{2}(m)}{m}=\frac{1}{3}\left[S_{1}^{3}(n)-S_{3}(n)\right]+S_{2,1}(n) .
$$

The evaluation of the more complicated sums can be found in Ref. [201].

\section{Appendix B. Method of "Projectors"}

In Refs. [24,26] we used a special case of the "projection" method [20,21] —is so-called "differentiation" method, which allows for a diagram depending on two momenta $p$ and $q$, where $p^{2}=0$, to obtain the coefficients for powers of $[2(p q)] / q^{2}$ (in the case of nonzero quark masses, similar canculations were done in Ref. [202]). These coefficients will be call the "moments".

As a first example, we consider the diagram $J_{1}(\alpha, q, p)$

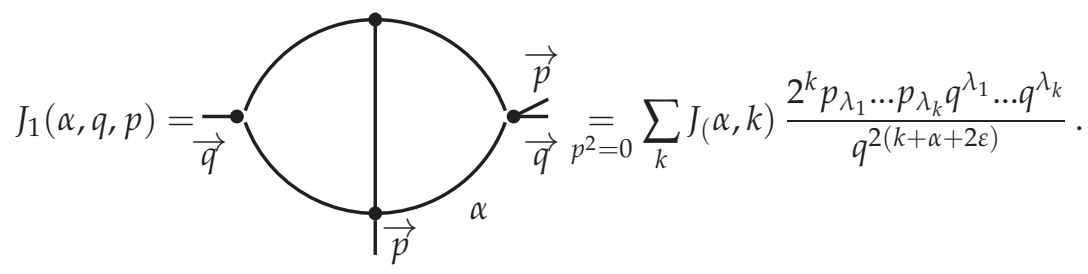

We differentiate expression (6) on the right and on the left $n$ times with respect to $p$ and set $p=0$. On the left we have

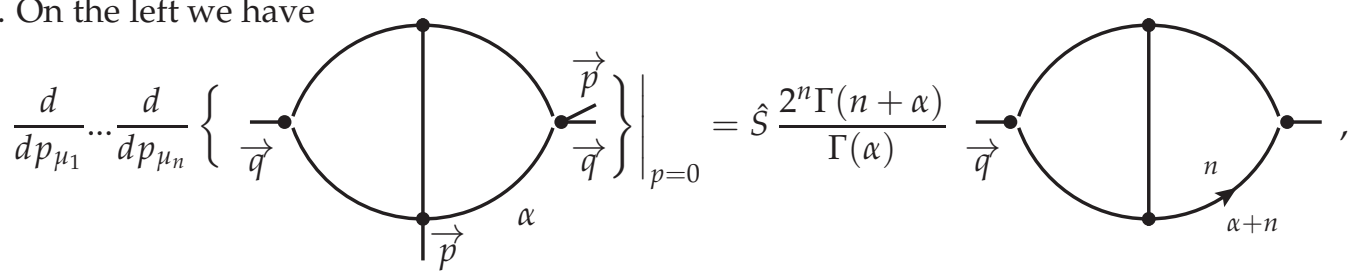

where $\hat{S}$ is a symmetrization on indeces: $\lambda_{i}, \mu_{j}(i=1, \ldots, m, j=1, \ldots, n)$. 
On the right we get

$$
\left.\sum_{k} J_{1}(\alpha, k) \frac{2^{k} q^{v_{1}} \ldots q^{v_{k}}}{q^{2(k+\alpha+2 \varepsilon)}} \frac{d}{d p_{\mu_{1}}} \ldots \frac{d}{d p_{\mu_{n}}}\left(p^{v_{1}} \ldots p^{v_{k}}\right)\right|_{p=0}=\hat{S} n ! J_{1}(\alpha, n) \frac{2^{n} q^{v_{1}} \ldots q^{v_{n}}}{q^{2(n+\alpha+2 \varepsilon)}} .
$$

Therefore, for the moment $J_{1}(\alpha, n)$ of the diagram we have the following expression:

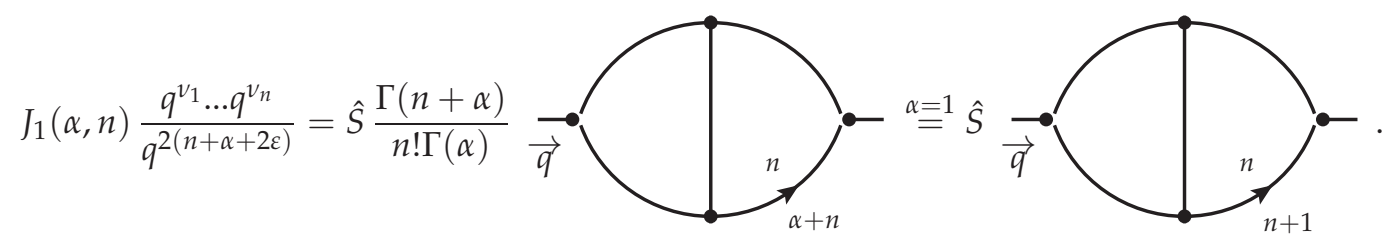

Next, we will neglect the symmetrizator $\hat{S}$.

Note that this transformation from the diagram to its moment remains valid for arbitrary indices of the diagram lines, as well as the presence of additional momentums in the propagators of the diagram (if the latter are located on a differentiable line, then some changes will be required).

The second consider diagram is

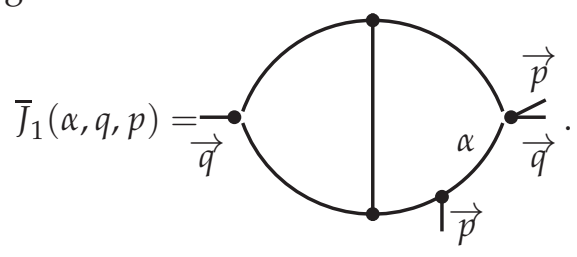

By analogy with the previous diagram we have for its moments:

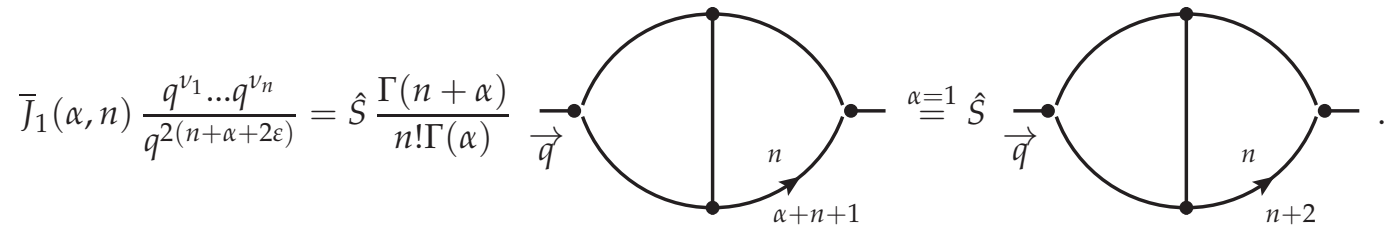

A similar conclusion can be drawn for the following diagram

$$
\hat{J}_{1}(\alpha, \beta, q, p)=\vec{q}
$$

Its moment has the form

$$
\hat{J}_{1}(\alpha, \beta, n) \frac{q^{v_{1}} \ldots q^{v_{n}}}{q^{2(n+\alpha+2 \varepsilon)}}=\sum_{k=0}^{n} \frac{\Gamma(k+\beta) \Gamma(n-k+\alpha)}{(n-k) ! k ! \Gamma(\alpha) \Gamma(\beta)}
$$

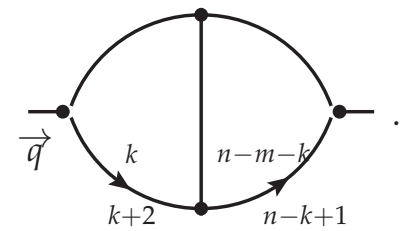

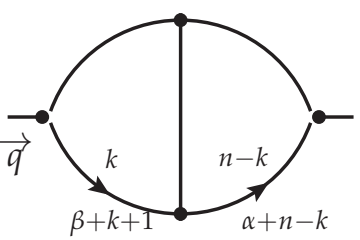


Note that there is another technique to calculate the considered diagrams: the "gluing" method [19]. Using the orthogonality of traceless product, on can obtain the moment of the diagram by an additional integration on the $q$ momentum with a propagator, which has an index $\delta$ and the additional tranceless product. This additional integration leads to very complicated three-loop diagrams. For the considered Feynman integrals $J_{1}(\alpha, q, p), \bar{J}_{1}(\alpha, q, p), \hat{J}_{1}(\alpha, \beta, q, p)$ these "gluing" three-loop diagrams have the following form
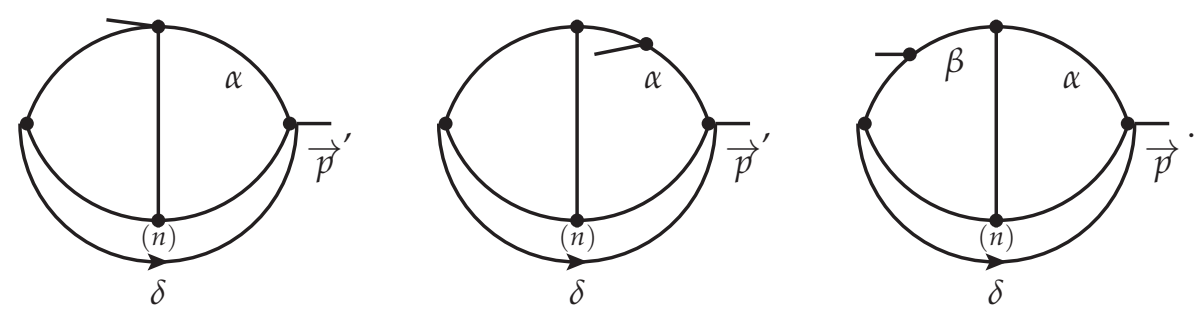

The evaluation of these complicated diagrams is above of the slope of the paper. Some example of application of the "gluing" method can be found in Ref. [24].

As a conclusion of Appendix B, we would like to note, when applying the method of "projectors" [20,21], the expression obtained for the $n$th moment of the initial diagram has alwais much simpler form than using the "gluing" method [19].

\section{Appendix C. About Analytic Continuation}

Here we demonstrate the direct calculation the particular case $C_{2}(1, n=0)$ from the general expression $C_{2}(1, n)$ in (122) by using an analytic continuation (from even $n$ values) of the sum

$$
S_{-2}(n)=\sum_{m=1}^{n} \frac{(-1)^{m}}{m^{2}}
$$

Indeed, using the simple sum, it is very convenient to show the basic steps of the analytic continuation itself. In the case of more general nested sums $S_{ \pm a, \pm b, \ldots}(n)$ formulas are more complex, which may make it difficult to understand the procedure.

The basic idea of the analytic continuation is very simple: try to translate the argument $n$ from the upper limit of the sum to the summed expression. After the procedure performed we have a possibility to expand and to differenciate with respect of $n$ and so on.

Firstly we represent the sum $S_{-2}(n)$ in (A23) as

$$
S_{-2}(n)=\left(\sum_{m=1}^{\infty}-\sum_{m=n+1}^{\infty}\right) \frac{(-1)^{m}}{m^{2}}=S_{-2}(\infty)-(-1)^{n} \sum_{m=1}^{\infty} \frac{(-1)^{m}}{(m+n)^{2}} .
$$

and we see the unpleasant factor $(-1)^{n}$ in the front of the last term in r.h.s.

Considering the variable $(-1)^{n} S_{-2}(n)$ :

$$
(-1)^{n} S_{-2}(n)=(-1)^{n} S_{-2}(\infty)-\sum_{m=1}^{\infty} \frac{(-1)^{m}}{(m+n)^{2}}
$$

we move the factor $(-1)^{n}$ to the front of the first term.

Now we introduce the new $n$-dependent function $\bar{S}_{-2}(n)$ as

$$
\bar{S}_{-2}(n)=(-1)^{n} S_{-2}(n)+\left(1-(-1)^{n}\right) S_{-2}(\infty),
$$


which coincides with the initial one $S_{-2}(n)$ at even $n$ values and have no the unpleasant factor $(-1)^{n}$

$$
\bar{S}_{-2}(n)=S_{-2}(\infty)-\sum_{m=1}^{\infty} \frac{(-1)^{m}}{(m+n)^{2}} .
$$

So, the function $\bar{S}_{-2}(n)$ can be considered as the analytic continuation (from even $n$ values) of the sum $S_{-2}(n)$.

Now it is possible to consider the small- $n$ limit of $C_{2}(1, n)$ using the corresponding limit of $\bar{S}_{-2}(n)$ as

$$
\begin{aligned}
\bar{S}_{-2}(n=\delta \rightarrow 0) & =S_{-2}(\infty)-\sum_{m=1}^{\infty} \frac{(-1)^{m}}{m^{2}}\left[1-2 \frac{\delta}{m}+O\left(\delta^{2}\right)\right] \\
& =2 \delta \sum_{m=1}^{\infty} \frac{(-1)^{m}}{m^{3}}+O\left(\delta^{2}\right)=2 \delta S_{-3}(\infty)+O\left(\delta^{2}\right) .
\end{aligned}
$$

In r.h.s the function $S_{-3}(\infty)$ coincides with the Euler number $\bar{\zeta}_{3}$, where

$$
\bar{\zeta}_{a}=\sum_{m=1}^{\infty} \frac{(-1)^{m}}{m^{a}}=\left(\frac{1}{2^{a}}-1\right) \zeta_{a}=-\frac{3}{4} \zeta_{3} \text { for } a=3 .
$$

Thus, in the small- $n$ limit we have for $\bar{S}_{-2}(n)$ :

$$
\bar{S}_{-2}(n=\delta \rightarrow 0)=-\frac{3}{4} \zeta_{3} \delta+O\left(\delta^{2}\right)
$$

and for the coefficient function $C_{2}(1, n=0)$ of (122)

$$
C_{2}(1, n=0)=-\frac{4}{\delta(1+\delta)} \bar{S}_{-2}(n=\delta \rightarrow 0)=6 \zeta_{3}+O(\delta),
$$

that exactly coincides with $C_{1}(1, n=0)$.

This analytic continuation has many important uses. For example, to analyze the evolution of parton distributions and DIS structure functions, there is a popular approach [203] which is based on the Gegenbauer polynomials, which in-turn are associated with the moments of parton distributions and structure functions. Using in the analysis a simple evolution for moments, which is determined here by the simple differential DGLAP equations, at the last step the parton distributions and/or structure functions are restored by summing (to a certain value $N_{\mathrm{MAX}}$ ) the Gegenbauer polynomials.

In this analysis, evolution should be performed for both even and odd moments, so the analytic continuation is necessary. Using this analytical continuation, a lot of analysis of experimental data was performed (see a review in [204]) by the method described here.

Another important application [205,206] is the study of parton distributions and structure functions in the region of small values of the Bjerken variable $x$, which directly relates with above study of the nested sums at $n \rightarrow 0$. The approch includes, in particular, an extraction of gluon density and longitudinal structure function from data for structural function $F_{2}$, the evolution of parton distributions at low $x$ in nucleon and in nuclei, an ultrahigh-energy asymptotics of the neutrino-hadron interaction cross section. Some review of these studies can be found in Ref. [207].

\section{References}

1. Peterman, A. Renormalization Group and the Deep Structure of the Proton. Phys. Rept. 1979, 53, 157. [CrossRef]

2. 't Hooft, G.; Veltman, M.J.G. Regularization and Renormalization of Gauge Fields. Nucl. Phys. B 1972, 44, 189. [CrossRef] 
3. Bollini, C.G.; Giambiagi, J.J. Dimensional Renormalization: The Number of Dimensions as a Regularizing Parameter. Nuovo Cim. B 1972, 12, 20.

4. Cicuta, G.M.; Montaldi, E. Analytic renormalization via continuous space dimension. Lett. Nuovo Cim. 1972, 4, 329. [CrossRef]

5. 't Hooft, G. Dimensional regularization and the renormalization group. Nucl. Phys. B 1973, 61, 455. [CrossRef]

6. D'Eramo, M.; Parisi, G.; Peliti, L. Theoretical Predictions For Critical Exponents At The Lambda Point of Bose Liquids. Lett. Nuovo Cim. 1971, 2, 878. [CrossRef]

7. Vasiliev, A.N.; Pismak, Y.M.; Khonkonen, Y.R. 1/N Expansion: Calculation Of The Exponents Eta And Nu In The Order 1/N**2. For Arbitrary Number Of Dimensions. Theor. Math. Phys. 1981, 47, 465. [CrossRef]

8. Kazakov, D.I. The Method Of Uniqueness, A New Powerful Technique For Multiloop Calculations. Phys. Lett. B 1983, 133, 406. [CrossRef]

9. Kazakov, D.I. Calculation Of Feynman Integrals By The Method Of 'uniqueness'. Theor. Math. Phys. 1984, 58, 223. [CrossRef]

10. Usyukina, N.I. Calculation Of Many Loop Diagrams Of Perturbation Theory. Theor. Math. Phys. 1983, 54, 78. [CrossRef]

11. Belokurov, V.V.; Usyukina, N.I. Calculation Of Ladder Diagrams In Arbitrary Order. J. Phys. A 1983, 16 2811. [CrossRef]

12. Belokurov, V.V.; Usyukina, N.I. An Algorithm For Calculating Massless Feynman Diagrams. Theor. Math. Phys. 1989, 79, 385.

13. Kazakov, D.I. Multiloop Calculations: Method of Uniqueness and Functional Equations. Theor. Math. Phys. 1985, 62, 84. [CrossRef]

14. Kotikov, A.V. The Gegenbauer polynomial technique: The Evaluation of a class of Feynman diagrams. Phys. Lett. B 1996, 375, 240. [CrossRef]

15. Kotikov, A.V.; Teber, S. New Results for a Two-Loop Massless Propagator-Type Feynman Diagram. Theor. Math. Phys. 2018, 194, 284. [CrossRef]

16. Kotikov, A.V.; Teber, S. Multi-loop techniques for massless Feynman diagram calculations. Phys. Part. Nucl. 2019, 50, 1. [CrossRef]

17. Ryder, L.H. Quantum Field Theory; Cambridge University Press: Cambridge, UK, 1996.

18. Davydychev, A.I.; Tausk, J.B. A Magic connection between massive and massless diagrams. Phys. Rev. D 1996, 53, 7381. [CrossRef]

19. Chetyrkin, K.G.; Tkachov, F.V.; Gorishnii, S.G. Operator Product Expansion in the Minimal Subtraction Scheme. Phys. Lett. B 1982, 119, 407. [CrossRef]

20. Gorishnii, S.G.; Larin, S.A.; Tkachov, F.V. The Algorithm for Ope Coefficient Functions in the Ms Scheme. Phys. Lett. B 1983, 124, 217. [CrossRef]

21. Gorishnii, S.G.; Larin, S.A. Coefficient Functions of Asymptotic Operator Expansions in Minimal Subtraction Scheme. Nucl. Phys. B 1987, 283, 452. [CrossRef]

22. Tkachov, F.V. On The Operator Product Expansion In The Ms Scheme. Phys. Lett. B 1983, 124, 212. [CrossRef]

23. Chetyrkin, K.G. Infrared $R^{*}$-Operation and operator product expansion in the minimal subtraction scheme. Phys. Lett. B 1983, 126, 371. [CrossRef]

24. Kazakov, D.I.; Kotikov, A.V. The Method of Uniqueness: Multiloop Calculations in QCD. Theor. Math. Phys. 1988, 73, 1264. [CrossRef]

25. Kazakov, D.I.; Kotikov, A.V. Total $\alpha^{-}$s Correction to Deep Inelastic Scattering Cross-section Ratio, $\mathrm{R}=$ $\sigma^{-} 1 / \sigma^{-} \mathrm{t}$ in QCD. Calculation of Longitudinal Structure Function. Nucl. Phys. B 1988, 307, 721; Erratum in 1990, 345, 299. [CrossRef]

26. Kotikov, A.V. The Calculation of Moments of Structure Function of Deep Inelastic Scattering in QCD. Theor. Math. Phys. 1989, 78, 134. [CrossRef]

27. Kazakov, D.I.; Kotikov, A.V. On the value of the alpha-s correction to the Callan-Gross relation Phys. Lett. B 1992, 291, 171. [CrossRef]

28. Kotikov, A.V. New method of massive Feynman diagrams calculation. Mod. Phys. Lett. A 1991, 6, 677. [CrossRef]

29. Kotikov, A.V. New method of massive Feynman diagrams calculation. Vertex type functions. Int. J. Mod. Phys. A 1992, 7, 1977. [CrossRef] 
30. Henn J.M.; Plefka, J.C. Scattering Amplitudes in Gauge Theories; Lecture Notes in Physics; Springer: Berlin/Heidelberg, Germany, 2014; Volume 883.

31. Tarasov, O.V. Connection between Feynman integrals having different values of the space-time dimension. Phys. Rev. D 1996, 54, 6479. [CrossRef]

32. Tarasov, O.V. Generalized recurrence relations for two loop propagator integrals with arbitrary masses. Nucl. Phys. B 1997, 502, 455. [CrossRef]

33. Lee, R.N. Presenting LiteRed: A tool for the Loop InTEgrals REDuction. arXiv 2012, arXiv:1212.2685.

34. Lee, R.N. LiteRed 1.4: A powerful tool for reduction of multiloop integrals. J. Phys. Conf. Ser. 2014, 523, 012059. [CrossRef]

35. Lee, R.N.; Smirnov, A.V.; Smirnov, V.A. Analytic Results for Massless Three-Loop Form Factors. J. High Energy Phys. 2010, 1004, 201020. [CrossRef]

36. Kotikov, A.V.; Lipatov, L.N. NLO corrections to the BFKL equation in QCD and in supersymmetric gauge theories. Nucl. Phys. B 2000, 582, 19. [CrossRef]

37. Lipatov, L.N. Reggeization of the Vector Meson and the Vacuum Singularity in Nonabelian Gauge Theories. Sov. J. Nucl. Phys. 1976, 23, 338.

38. Fadin, V.S.; Kuraev, E.A.; Lipatov, L.N. On the Pomeranchuk Singularity in Asymptotically Free Theories. Phys. Lett. B 1975, 60, 50. [CrossRef]

39. Kuraev, E.A.; Lipatov, L.N.; Fadin, V.N. Multi - Reggeon Processes in the Yang-Mills Theory. Sov. Phys. JETP 1976, 44, 443.

40. Kuraev, E.A.; Lipatov, L.N.; Fadin, V.N. The Pomeranchuk Singularity in Nonabelian Gauge Theories. Sov. Phys. JETP 1977, 45, 199.

41. Balitsky, I.I.; Lipatov, L.N. The Pomeranchuk Singularity in Quantum Chromodynamics. Sov. J. Nucl. Phys. 1978, 28, 822.

42. Balitsky, I.I.; Lipatov, L.N. Calculation Of Meson Meson Interaction Cross-section In Quantum Chromodynamics. JETP Lett. 1979, 30, 355.

43. Fadin, V.S.; Lipatov, L.N. BFKL pomeron in the next-to-leading approximation. Phys. Lett. B 1998, 429127. [CrossRef]

44. Camici, G.; Ciafaloni, M. Energy scale(s) and next-to-leading BFKL equation. Phys. Lett. B 1998, 430, 349.

45. Brink, L.; Schwarz, J.H.; Scherk, J. Supersymmetric Yang-Mills Theories. Nucl. Phys. B 1977, $121,77$. [CrossRef]

46. Gliozzi, F.; Scherk, J.; Olive, D.I. Supersymmetry, Supergravity Theories and the Dual Spinor Model. Nucl. Phys. B 1977, 122, 253. [CrossRef]

47. Kotikov, A.V.; Lipatov, L.N. DGLAP and BFKL equations in the $\mathcal{N}=4$ supersymmetric gauge theory. Nucl. Phys. B 2003, 661, 19. [CrossRef]

48. Kotikov, A.V.; Lipatov, L.N. DGLAP and BFKL evolution equations in the $\mathcal{N}=4$ supersymmetric gauge theory. arXiv 2001, arXiv:hep-ph/0112346.

49. Kotikov, A.V.; Lipatov, L.N.; Velizhanin, V.N. Anomalous dimensions of Wilson operators in $\mathcal{N}=4 \mathrm{SYM}$ theory. Phys. Lett. B 2003, 557, 114. [CrossRef]

50. Kotikov, A.V.; Lipatov, L.N.; Onishchenko, A.I.; Velizhanin, V.N. Three loop universal anomalous dimension of the Wilson operators in $\mathcal{N}=4$ SUSY Yang-Mills model. Phys. Lett. B 2004, 595, 521. [CrossRef]

51. Bianchi, L.; Forini, V.; Kotikov, A.V. On DIS Wilson coefficients in $\mathcal{N}=4$ super Yang-Mills theory. Phys. Lett. B 2013, 725, 394. [CrossRef]

52. Moch, S.; Vermaseren, J.A.M.; Vogt, A. The Three loop splitting functions in QCD: The Nonsinglet case. Nucl. Phys. B 2004, 688, 101. [CrossRef]

53. Vogt, A.; Moch, S.; Vermaseren, J.A.M. The Three-loop splitting functions in QCD: The Singlet case. Nucl. Phys. B 2004, 691, 129. [CrossRef]

54. Vermaseren, J.A.M.; Vogt A.; Moch, S. The Third-order QCD corrections to deep-inelastic scattering by photon exchange. Nucl. Phys. B 2005, 724, 3. [CrossRef]

55. Broadhurst, D.J. The master two loop diagram with masses. Z. Phys. C 1990, 47, 115. [CrossRef]

56. Fleischer, J.; Kotikov, A.V.; Veretin, O.L. Analytic two loop results for selfenergy type and vertex type diagrams with one nonzero mass. Nucl. Phys. B 1999, 547, 343.

57. Fleischer, J.; Kotikov, A.V.; Veretin, O.L. Applications of the large mass expansion. Acta Phys. Polon. B 1998, $29,2611$. 
58. Eden, B.; Heslop, P.; Korchemsky, G.P.; Sokatchev, E. Hidden symmetry of four-point correlation functions and amplitudes in $\mathcal{N}=4$ SYM. Nucl. Phys. B 2012, 862, 193. [CrossRef]

59. Dixon, L.J. Scattering amplitudes: The most perfect microscopic structures in the universe. J. Phys. A 2011, 44, 454001. [CrossRef]

60. Dixon, L.J.; Drummond, J.M.; Henn, J.M. Analytic result for the two-loop six-point NMHV amplitude in N = 4 super Yang-Mills theory. J. High Energy Phys. 2012, 1201, 024. [CrossRef]

61. Brandhuber, A.; Travaglini, G.; Yang, G. Analytic two-loop form factors in $\mathcal{N}=4$ SYM. J. High Energy Phys. 2012, 1205, 082. [CrossRef]

62. Henn, J.M.; Moch, S.; Naculich, S.G. Form factors and scattering amplitudes in $\mathcal{N}=4$ SYM in dimensional and massive regularizations. J. High Energy Phys. 2011, 1112, 024. [CrossRef]

63. Schlotterer, O.; Stieberger, S. Motivic Multiple Zeta Values and Superstring Amplitudes. J. Phys. A 2013, 46, 475401. [CrossRef]

64. Broedel, J.; Schlotterer, O.; Stieberger, S. Polylogarithms, Multiple Zeta Values and Superstring Amplitudes. Fortsch. Phys. 2013, 61, 812. [CrossRef]

65. Stieberger, S.; Taylor, T.R. Maximally Helicity Violating Disk Amplitudes, Twistors and Transcendental Integrals. Phys. Lett. B 2012, 716, 236. [CrossRef]

66. Eden, B. Three-loop universal structure constants in $\mathcal{N}=4$ susy Yang-Mills theory. arXiv 2012, arXiv:1207.3112.

67. Ambrosio, R.G.; Eden, B.; Goddard, T.; Heslop, P.; Taylor, C. Local integrands for the five-point amplitude in planar $\mathcal{N}=4$ SYM up to five loops. J. High Energy Phys. 2015, 1501, 116. [CrossRef]

68. Chicherin, D.; Doobary, R.; Eden, B.; Heslop, P.; Korchemsky, G.P.; Sokatchev, E. Bootstrapping correlation functions in $\mathcal{N}=4$ SYM. J. High Energy Phys. 2016, 1603, 031. [CrossRef]

69. Eden, B.; Sfondrini, A. Three-point functions in $\mathcal{N}=4$ SYM: The hexagon proposal at three loops. J. High Energy Phys. 2016, 1602, 165. [CrossRef]

70. Basso, B.; Dixon, L.J.; Papathanasiou, G. The Origin of the Six-Gluon Amplitude in Planar $\mathcal{N}=4$ SYM. Phys. Rev. Lett. 2020, 124, 161603. [CrossRef]

71. Caron-Huot, S.; Dixon, L.J.; Dulat, F.; von Hippel, M.; McLeod, A.J.; Papathanasiou, G. Six-Gluon amplitudes in planar $\mathcal{N}=4$ super-Yang-Mills theory at six and seven loops. J. High Energy Phys. 2019, 1908, 016. [CrossRef]

72. Caron-Huot, S.; Dixon, L.J.; Dulat, F.; von Hippel, M.; McLeod, A.J.; Papathanasiou, G. The Double Pentaladder Integral to All Orders. J. High Energy Phys. 2018, 1807, 170. [CrossRef]

73. Caron-Huot, S.; Dixon, L.J.; McLeod, A.J.; von Hippel, M. Bootstrapping a Five-Loop Amplitude Using Steinmann Relations. Phys. Rev. Lett. 2016, 117, 241601. [CrossRef] [PubMed]

74. Abreu, S.; Dixon, L.J.; Herrmann, E.; Page, B.; Zeng, M. The two-loop five-point amplitude in $\mathcal{N}=8$ supergravity. J. High Energy Phys. 2019, 1903, 123. [CrossRef]

75. Abreu, S.; Dixon, L.J.; Herrmann, E.; Page, B.; Zeng, M. The two-loop five-point amplitude in $\mathcal{N}=4$ super-Yang-Mills theory. Phys. Rev. Lett. 2019, 122, 121603. [CrossRef] [PubMed]

76. Dixon, L.J.; Drummond, J.M.; Harrington, T.; McLeod, A.J.; Papathanasiou, G.; Spradlin, M. Heptagons from the Steinmann Cluster Bootstrap. J. High Energy Phys. 2017, 1702, 137. [CrossRef]

77. Dixon, L.J.; von Hippel, M.; McLeod, A.J.; Trnka, J. Multi-loop positivity of the planar $\mathcal{N}=4$ SYM six-point amplitude. J. High Energy Phys. 2017, 1702, 112. [CrossRef]

78. Dixon, L.J.; von Hippel, M.; McLeod, A.J. The four-loop six-gluon NMHV ratio function. J. High Energy Phys. 2016, 1601, 053. [CrossRef]

79. Dixon, L.J.; Drummond, J.M.; Duhr, C.; Pennington, J. The four-loop remainder function and multi-Regge behavior at NNLLA in planar $\mathcal{N}=4$ super-Yang-Mills theory. J. High Energy Phys. 2014, 1406, 116. [CrossRef]

80. Badger, S.; Chicherin, D.; Gehrmann, T.; Heinrich, G.; Henn, J.M.; Peraro, T.; Wasser, P.; Zhang, Y.; Zoia, S. Analytic form of the full two-loop five-gluon all-plus helicity amplitude. Phys. Rev. Lett. 2019, 123, 071601. [CrossRef]

81. Chicherin, D.; Gehrmann, T.; Henn, J.M.; Wasser, P.; Zhang, Y.; Zoia, S. The two-loop five-particle amplitude in $\mathcal{N}=8$ supergravity. J. High Energy Phys. 2019, 1903, 115. [CrossRef]

82. Chicherin, D.; Gehrmann, T.; Henn, J.M.; Wasser, P.; Zhang, Y.; Zoia, S. Analytic result for a two-loop five-particle amplitude. Phys. Rev. Lett. 2019, 122, 121602. [CrossRef] 
83. Chicherin, D.; Gehrmann, T.; Henn, J.M.; Lo Presti, N.A.; Mitev, V.; Wasser, P. Analytic result for the nonplanar hexa-box integrals. J. High Energy Phys. 2019, 1903, 042. [CrossRef]

84. Chicherin, D.; Henn, J.M.; Mitev, V. Bootstrapping pentagon functions. J. High Energy Phys. 2018, $1805,164$. [CrossRef]

85. Gehrmann, T.; Henn, J.M.; Lo Presti, N.A. Pentagon functions for massless planar scattering amplitudes. J. High Energy Phys. 2018, 1810, 103. [CrossRef]

86. Bianchi, L.; Brandhuber, A.; Panerai, R.; Travaglini, C. Dual conformal invariance for form factors. J. High Energy Phys. 2019, 1902, 134. [CrossRef]

87. Bianchi, L.; Brandhuber, A.; Panerai, R.; Travaglini, C. Form factor recursion relations at loop level. J. High Energy Phys. 2019, 1902, 182. [CrossRef]

88. Brandhuber, A.; Kostacinska, M.; Penante, B.; Travaglini, C. Higgs amplitudes from $\mathcal{N}=4$ super Yang-Mills theory. Phys. Rev. Lett. 2017, 119, 161601. [CrossRef]

89. Brandhuber, A.; Hughes, E.; Panerai, R.; Spence, B.; Travaglini, C. The connected prescription for form factors in twistor space. J. High Energy Phys. 2016, 1611, 143. [CrossRef]

90. Brandhuber, A.; Kostacinska, M.; Penante, B.; Travaglini, C.; Young, D. The SU(2।3) dynamic two-loop form factors. J. High Energy Phys. 2016, 1608, 134. [CrossRef]

91. Brandhuber, A.; Heslop, P.; Travaglini, C.; Young, D. Yangian Symmetry of Scattering Amplitudes and the Dilatation Operator in $N=4$ Supersymmetric Yang-Mills Theory. Phys. Rev. Lett. 2015, 115, 141602. [CrossRef]

92. Caron-Huot, S.; Chicherin, D.; Henn, J.M.; Zhang, Y.; Zoia, S. Multi-Regge Limit of the Two-Loop Five-Point Amplitudes in $\mathcal{N}=4$ Super Yang-Mills and $\mathcal{N}=8$ Supergravity. arXiv 2020, arXiv:2003.03120.

93. Henn, J.; Power, B.; Zoia, S. Conformal Invariance of the One-Loop All-Plus Helicity Scattering Amplitudes. J. High Energy Phys. 2020, 2002, 019. [CrossRef]

94. Chicherin, D.; Henn, J.M.; Sokatchev, E. Implications of nonplanar dual conformal symmetry. J. High Energy Phys. 2018, 1809, 012. [CrossRef]

95. Henn, J.; Herrmann, E.; Parra-Martinez, J. Bootstrapping two-loop Feynman integrals for planar $\mathcal{N}=4$ sYM. J. High Energy Phys. 2018, 1810, 059. [CrossRef]

96. Henn, J.M.; Mistlberger, B. Four-graviton scattering to three loops in $\mathcal{N}=8$ supergravity. J. High Energy Phys. 2019, 1905, 023. [CrossRef]

97. Fotopoulos, A.; Stieberger, S.; Taylor, T.R.; Zhu, B. Extended BMS Algebra of Celestial CFT. J. High Energy Phys. 2020, 2003, 130. [CrossRef]

98. Stieberger, S.; Taylor, T.R. Symmetries of Celestial Amplitudes. Phys. Lett. B 2019, 793, 141 [CrossRef]

99. Stieberger, S.; Taylor, T.R. New relations for Einstein-Yang-Mills amplitudes. Nucl. Phys. B 2016, 913, 151. [CrossRef]

100. Stieberger, S.; Taylor, T.R. Subleading terms in the collinear limit of Yang-Mills amplitudes. Phys. Lett. B 2015, 750, 587. [CrossRef]

101. Puhlfürst, G.; Stieberger, S. Differential Equations, Associators, and Recurrences for Amplitudes. Nucl. Phys. B 2016, 902, 186. [CrossRef]

102. De Leeuw, M.; Eden, B.; Le Plat, D.; Meier, T.; Sfondrini, A. Multi-particle finite-volume effects for hexagon tessellations. arXiv 2019, arXiv:1912.12231.

103. Eden, B.; Jiang, Y.; de Leeuw, M.; Meier, T.; le Plat, D.; Sfondrini, A. Positivity of hexagon perturbation theory. J. High Energy Phys. 2018, 1811, 097. [CrossRef]

104. Eden, B.; Jiang, Y.; le Plat, D.; Sfondrini, A. Colour-dressed hexagon tessellations for correlation functions and non-planar corrections. J. High Energy Phys. 2018, 1802, 170. [CrossRef]

105. Eden, B.; Heslop, P.; Mason, L. The Correlahedron. J. High Energy Phys. 2017, 1709, 156. [CrossRef]

106. Eden, B.; Sfondrini, A. Tessellating cushions: Four-point functions in $\mathcal{N}=4$ SYM. J. High Energy Phys. 2017, 1710, 098. [CrossRef]

107. Eden B.; Smirnov, V.A. Evaluating four-loop conformal Feynman integrals by D-dimensional differential equations. J. High Energy Phys. 2016, 1610, 115. [CrossRef]

108. Kotikov, A.V. The Property of maximal transcendentality in the $\mathcal{N}=4$ Supersymmetric Yang-Mills. In Subtleties in Quantum Field Theory; Diakonov, D., Ed.; PNPI: Gatchina, Russia, 2010; pp. 150-174.

109. Kotikov, A.V. The property of maximal transcendentality: Calculation of anomalous dimensions in the $\mathcal{N}$ = 4 SYM and master integrals. Phys. Part. Nucl. 2013, 44, 374. [CrossRef] 
110. Kotikov, A.V.; Onishchenko, A.I. DGLAP and BFKL equations in $\mathcal{N}=4$ SYM: From weak to strong coupling. arXiv 2019, arXiv:1908.05113.

111. Kotikov, A.V. The property of maximal transcendentality: Calculation of master integrals. Theor. Math. Phys. 2013, 176, 913. [CrossRef]

112. Kotikov, A.V. The property of maximal transcendentality: Calculation of Feynman integrals. Theor. Math. Phys. 2017, 190, 391. [CrossRef]

113. Chetyrkin, K.G.; Kataev, A.L.; Tkachov, F.V. New Approach to Evaluation of Multiloop Feynman Integrals: The Gegenbauer Polynomial x Space Technique. Nucl. Phys. B 1980, 174, 345. [CrossRef]

114. Broadhurst, D.J.; Kotikov, A.V. Compact analytical form for nonzeta terms in critical exponents at order 1/N**3. Phys. Lett. B 1990, 441, 345. [CrossRef]

115. Teber, S. Electromagnetic current correlations in reduced quantum electrodynamics. Phys. Rev. D 2012, 86, 025005. [CrossRef]

116. Kotikov, A.V.; Teber, S. Note on an application of the method of uniqueness to reduced quantum electrodynamics. Phys. Rev. D 2013, 87, 087701. [CrossRef]

117. Kotikov, A.V. Critical behavior of 3-D electrodynamics. JETP Lett. 1993, 58, 731.

118. Kotikov, A.V. On the Critical Behavior of (2+1)-Dimensional QED. Phys. Atom. Nucl. 2012, 75, 890. [CrossRef]

119. Kotikov, A.V.; Shilin, V.I.; Teber, S. Critical behavior of ( $2+1$ )-dimensional QED: $1 / \mathrm{N}_{f}$ corrections in the Landau gauge. Phys. Rev. D 2016, 94, 056009; Erratum in 2019, 99, 119901. [CrossRef]

120. Kotikov, A.V.; Teber, S. Critical behavior of $(2+1)$-dimensional QED: $1 / N_{f}$ corrections in an arbitrary nonlocal gauge. Phys. Rev. D 2016, 94, 114011; Addendum in 2019, 99, 059902. [CrossRef]

121. Kotikov, A.V.; Teber, S. Two-loop fermion self-energy in reduced quantum electrodynamics and application to the ultrarelativistic limit of graphene. Phys. Rev. D 2014, 89, 065038. [CrossRef]

122. Teber, S.; Kotikov, A.V. The method of uniqueness and the optical conductivity of graphene: New application of a powerful technique for multiloop calculations. Theor. Math. Phys. 2017, 190, 446. [CrossRef]

123. Chetyrkin, K.G.; Tkachov, F.V. Integration By Parts: The Algorithm To Calculate Beta Functions In 4 Loops. Nucl. Phys. B 1981, 192, 159. [CrossRef]

124. Tkachov, F.V. A Theorem On Analytical Calculability Of Four Loop Renormalization Group Functions. Phys. Lett. B 1981, 100, 65. [CrossRef]

125. Gorishnii, S.G.; Isaev, A.P. On an Approach to the Calculation of Multiloop Massless Feynman Integrals. Theor. Math. Phys. 1985, 62, 232. [CrossRef]

126. Broadhurst, D.J. Exploiting the 1.440 Fold Symmetry of the Master Two Loop Diagram. Z. Phys. C 1986, 32, 249. [CrossRef]

127. Kazakov, D.I. Analytical Methods For Multiloop Calculations: Two Lectures On The Method Of Uniqueness; JINR Publishing Department: Dubna, Russia, 1984.

128. Vermaseren, J.A.M. Harmonic sums, Mellin transforms and integrals. Int. J. Mod. Phys. A 1999, $14,2037$. [CrossRef]

129. Broadhurst, D.J. Dimensionally continued multiloop gauge theory. arXiv 1999, arXiv:hep-th/9909185.

130. Kotikov, A.V.; Teber, S. Landau-Khalatnikov-Fradkin transformation and the mystery of even $\zeta$-values in Euclidean massless correlators. Phys. Rev. D 2019, 100, 105017. [CrossRef]

131. Kotikov, A.V.; Teber, S. Landau-Khalatnikov-Fradkin transformation and hatted $\zeta$-values. arXiv 1999, arXiv:1912.10957.

132. Kniehl, B.A.; Kotikov, A.V.; Onishchenko, A.; Veretin, O. Two-loop sunset diagrams with three massive lines. Nucl. Phys. B 2006, 738, 306. [CrossRef]

133. Kniehl, B.A.; Kotikov, A.V.; Onishchenko, A.I.; Veretin, O.L. Two-loop diagrams in non-relativistic QCD with elliptics. Nucl. Phys. B 2019, 948, 114780. [CrossRef]

134. Kniehl, B.A.; Kotikov, A.V. Calculating four-loop tadpoles with one non-zero mass. Phys. Lett. B 2006, 638, 531. [CrossRef]

135. Kniehl, B.A.; Kotikov, A.V. Counting master integrals: Integration-by-parts procedure with effective mass. Phys. Lett. B 2012, 712, 233. [CrossRef]

136. Kotikov, A.V. Differential equations method: New technique for massive Feynman diagrams calculation. Phys. Lett. B 1991, 254, 158. [CrossRef] 
137. Kotikov, A.V. Differential equations method: The Calculation of vertex type Feynman diagrams. Phys. Lett. B 1991, 259, 314. [CrossRef]

138. Kotikov, A.V. Differential equation method: The Calculation of $\mathrm{N}$ point Feynman diagrams. Phys. Lett. $B$ 1991, 267, 123. [CrossRef]

139. Kotikov, A.V. New method of massive N point Feynman diagrams calculation. Mod. Phys. Lett. A 1991, 6, 3133. [CrossRef]

140. Remiddi, E. Differential equations for Feynman graph amplitudes. Nuovo Cim. A 1997, 110, 1435.

141. Fleischer, J.; Kalmykov, M.Y.; Kotikov, A.V. Two-loop self-energy master integrals on shell. Phys. Lett. B 1999, 462, 169. [CrossRef]

142. Fleischer, J.; Kotikov, A.V.; Veretin, O.L. The differential equation method: Calculation of vertex-type diagrams with one non-zero mass. Phys. Lett. B 1998, 417, 163. [CrossRef]

143. Kotikov, A.; Kuhn, J.H.; Veretin, O. Two-Loop Formfactors in Theories with Mass Gap and Z-Boson Production. Nucl. Phys. B 2008, 788, 47. [CrossRef]

144. Kniehl, B.A.; Kotikov, A.V.; Onishchenko, A.I.; Veretin, O.L. Strong-coupling constant with flavor thresholds at five loops in the MS-bar scheme. Phys. Rev. Lett. 2006, 97, 042001. [CrossRef]

145. Kniehl, B.A.; Kotikov, A.V.; Merebashvili, Z.V.; Veretin, O.L. Heavy-quark pair production in polarized photon-photon collisions at next-to-leading order: Fully integrated total cross sections. Phys. Rev. D 2009, 79, 114032. [CrossRef]

146. Kniehl, B.A.; Kotikov, A.V.; Veretin, O.L. Orthopositronium lifetime: Analytic results in $O(\alpha)$ and $O\left(\alpha^{3} \ln (\alpha)\right)$. Phys. Rev. Lett. 2008, 101, 193401. [CrossRef] [PubMed]

147. Kniehl, B.A; Kotikov, A.V.; Veretin, O.L. Orthopositronium lifetime at O(alpha) and $\mathrm{O}\left(\mathrm{alpha}^{3} \ln (\mathrm{alpha})\right)$ in closed form. Phys. Rev. A 2009, 80, 052501. [CrossRef]

148. Gehrmann, T.; Henn, J.M.; Huber, T. The three-loop form factor in $\mathcal{N}=4$ super Yang-Mills. J. High Energy Phys. 2012, 1203, 101. [CrossRef]

149. Henn, J.M. Multiloop integrals in dimensional regularization made simple. Phys. Rev. Lett. 2013, 110, 251601. [CrossRef]

150. Henn, J.M. Lectures on differential equations for Feynman integrals. J. Phys. A 2015, 48, 153001. [CrossRef]

151. Adams, L.; Weinzierl, S. The $\varepsilon$-form of the differential equations for Feynman integrals in the elliptic case. Phys. Lett. B 2018, 781, 270. [CrossRef]

152. Lee, R.N. Reducing differential equations for multiloop master integrals. J. High Energy Phys. 2015, 1504, 108. [CrossRef]

153. Lee, R.N. Symmetric $\epsilon$ - and $(\epsilon+1 / 2)$-forms and quadratic constraints in "elliptic" sectors. J. High Energy Phys. 2018, 1810, 176. [CrossRef]

154. Lee, R.N.; Onishchenko, A.I. $\epsilon$-regular basis for non-polylogarithmic multiloop integrals and total cross section of the process $e^{+} e^{-} \rightarrow 2(Q \bar{Q})$. J. High Energy Phys. 2019, 1912, 084.

155. Duhr, C.; Dulat, F.; Hirschi, V.; Mistlberger, B. Higgs production in bottom quark fusion: Matching the 4and 5-flavour schemes to third order in the strong coupling. arXiv 2020, arXiv2004.04752.

156. Duhr, C.; Dulat, F.; Mistlberger, B. The Drell-Yan cross section to third order in the strong coupling constant. arXiv 2020, arXiv:2001.07717.

157. Henn J.; Mistlberger, B.; Smirnov V.A.; Wasser, P. Constructing d-log integrands and computing master integrals for three-loop four-particle scattering. arXiv 2020, arXiv:2002.09492.

158. Frellesvig H.; Kudashkin K.; Wever, C. Two-Loop QCD-EW Master Integrals for Z Plus Jet Production at Large Transverse Momentum. arXiv 2020, arXiv:2002.07776.

159. Bissi, A.; Fardelli, G.; Georgoudis, A. Towards All Loop Supergravity Amplitudes on $A d S_{5} \times S^{5}$ arXiv 2020, arXiv:2002.04604.

160. Dlapa, C.; Henn, J.; Yan, K. Deriving canonical differential equations for Feynman integrals from a single uniform weight integral. arXiv 2020, arXiv:2002.02340.

161. Misiak, M.; Rehman, A.; Steinhauser, A. Towards $\bar{B} \rightarrow X_{s} \gamma$ at the NNLO in QCD without interpolation in $m_{c}$. arXiv 2020, arXiv:2002.01548.

162. Anastasiou, C.; Deutschmann, N.; Schweitzer, A. Quark mass effects in two-loop Higgs amplitudes. arXiv 2020, arXiv:2001.06295.

163. Czakon, M.L.; Niggetiedt, M. Exact quark-mass dependence of the Higgs-gluon form factor at three loops in QCD. arXiv 2020, arXiv:2001.03008. 
164. Duhr, C. Mathematical aspects of scattering amplitudes. arXiv 2020, arXiv:1411.7538.

165. Devoto, A.; Duke, D.W. Table of Integrals and Formulae for Feynman Diagram Calculations. Riv. Nuovo Cim. 1984, 7N6, 1. [CrossRef]

166. Remiddi, E.; Vermaseren, J.A.M. Harmonic polylogarithms. Int. J. Mod. Phys. A 2000, 15, 725. [CrossRef]

167. Davydychev, A.I.; Kalmykov, M.Y. Massive Feynman diagrams and inverse binomial sums. Nucl. Phys. B 2004, 699, 3. [CrossRef]

168. Gribov, V.N.; Lipatov, L.N. Deep inelastic e p scattering in perturbation theory. Sov. J. Nucl. Phys. 1972, 15,438

169. Gribov, V.N.; Lipatov, L.N. e+ e- pair annihilation and deep inelastic e p scattering in perturbation theory. Sov. J. Nucl. Phys. 1972, 15, 675.

170. Lipatov, L.N. The parton model and perturbation theory. Sov. J. Nucl. Phys. 1975, $20,94$.

171. Altarelli, G.; Parisi, G. Asymptotic Freedom in Parton Language. Nucl. Phys. B 1977, 126, 298. [CrossRef]

172. Dokshitzer, Y.L. Calculation of the Structure Functions for Deep Inelastic Scattering and e+ e- Annihilation by Perturbation Theory in Quantum Chromodynamics. Sov. Phys. JETP 1977, 46, 641.

173. Lipatov, L.N. Next-to-leading corrections to the BFKL equation and the effective action for high energy processes in QCD. Nucl. Phys. Proc. Suppl. 2001, 99A, 175. [CrossRef]

174. Kotikov, A.V. Gluon distribution for small x. Phys. At. Nucl. 1994, 57, 133.

175. Kotikov, A.V.; Velizhanin, V.N. Analytic continuation of the Mellin moments of deep inelastic structure functions. arXiv 2005, arXiv:hep-ph/0501274.

176. Kotikov, A.V.; Lipatov, L.N.; Rej, A.; Staudacher, M.; Velizhanin, V.N. Dressing and wrapping. J. Stat. Mech. 2007, 0710, P10003. [CrossRef]

177. Bajnok, Z.; Janik, R.A.; Lukowski, T. Four-loop perturbative Konishi from strings and finite size effects for multiparticle states. Nucl. Phys. B 2009, 816, 376. [CrossRef]

178. Kotikov, A.V.; Rej, A.; Zieme, S. Analytic three-loop Solutions for $\mathcal{N}=4$ SYM Twist Operators. Nucl. Phys. B 2009, 813, 460. [CrossRef]

179. Beccaria, M.; Belitsky, A.V.; Kotikov, A.V.; Zieme, S. Analytic solution of the multiloop Baxter equation. Nucl. Phys. B 2010, 827, 565. [CrossRef]

180. Lukowski T.; Rej A.; Velizhanin, V.N. Five-Loop Anomalous Dimension of Twist-Two Operators. Nucl. Phys. B 2010, 831, 105. [CrossRef]

181. Marboe, C.; Velizhanin V.N.; Volin, D. Six-loop anomalous dimension of twist-two operators in planar $\mathcal{N}=4$ SYM theory. J. High Energy Phys. 2015, 1507, 084. [CrossRef]

182. Marboe, C.; Velizhanin, V. Twist-2 at seven loops in planar $\mathcal{N}=4$ SYM theory: Full result and analytic properties. J. High Energy Phys. 2016, 1611, 013. [CrossRef]

183. Staudacher, M. The Factorized S-matrix of CFT/AdS. J. High Energy Phys. 2005, 0505, 054; [CrossRef]

184. Beisert, N.; Staudacher, M. Long-range psu(2,2|4) Bethe Ansatze for gauge theory and strings. Nucl. Phys. B 2005, 727, 1. [CrossRef]

185. Beccaria, M. Three loop anomalous dimensions of twist-3 gauge operators in $\mathcal{N}=4$ SYM. J. High Energy Phys. 2007, 0709, 023. [CrossRef]

186. Beccaria, M.; Forini, V.; Lukowski, T.; Zieme, S. Twist-three at five loops, Bethe Ansatz and wrapping. J. High Energy Phys. 2009, 0903, 129. [CrossRef]

187. Velizhanin, V.N. Six-Loop Anomalous Dimension of Twist-Three Operators in $\mathcal{N}=4$ SYM. J. High Energy Phys. 2010, 1011, 129. [CrossRef]

188. W. Siegel, Supersymmetric Dimensional Regularization via Dimensional Reduction. Phys. Lett. B 1979, 84, 193. [CrossRef]

189. Maldacena, J.M. The Large N limit of superconformal field theories and supergravity. Adv. Theor. Math. Phys. 1998, 2, 231; Erratum in 1999, 38, 1113 [CrossRef]

190. Gubser, S.S.; Klebanov, I.R.; Polyakov, A.M. Gauge theory correlators from noncritical string theory. Phys. Lett. B 1998, 428, 105. [CrossRef]

191. Witten, E. Anti-de Sitter space and holography. Adv. Theor. Math. Phys. 1998, 2, 253. [CrossRef]

192. Kotikov, A.V.; Lipatov, L.N. On the highest transcendentality in $\mathcal{N}=4$ SUSY. Nucl. Phys. B 2007, $769,217$. [CrossRef]

193. Benna, M.K.; Benvenuti, S.; Klebanov I.R.; Scardicchio, A. A Test of the AdS/CFT correspondence using high-spin operators. Phys. Rev. Lett. 2007, 98, 131603. [CrossRef] 
194. Basso, B.; Korchemsky, G.P.; Kotanski, J. Cusp anomalous dimension in maximally supersymmetric Yang-Mills theory at strong coupling. Phys. Rev. Lett. 2008, 100, 091601. [CrossRef]

195. Basso, B.; Korchemsky, G.P. Embedding nonlinear O(6) sigma model into $\mathcal{N}=4$ super-Yang-Mills theory. Nucl. Phys. B 2009, 807, 397. [CrossRef]

196. Beisert, N.; Eden, B.; Staudacher, M. Transcendentality and Crossing. J. Stat. Mech. 2007, $0701, \mathrm{P} 01021$. [CrossRef]

197. Brower, R.C.; Polchinski, J.; Strassler, M.J.; Tan, C.T. The Pomeron and gauge/string duality. J. High Energy Phys. 2007, 0712, 005. [CrossRef]

198. Costa, M.S.; Goncalves, V.; Penedones, J. Conformal Regge theory. J. High Energy Phys. 2012, $1212,091$. [CrossRef]

199. Kotikov, A.V.; Lipatov, L.N. Pomeron in the $\mathcal{N}=4$ supersymmetric gauge model at strong couplings. Nucl. Phys. B 2013, 874, 889. [CrossRef]

200. Gromov, N.; Levkovich-Maslyuk, F.; Sizov, G.; Valatka, S. Quantum spectral curve at work: From small spin to strong coupling in $\mathcal{N}=4$ SYM. J. High Energy Phys. 2014, 1407, 156. [CrossRef]

201. Blumlein, J.; Broadhurst, D.J.; Vermaseren, J.A.M. The Multiple Zeta Value Data Mine. Comput. Phys. Commun. 2010, 181, 582. [CrossRef]

202. Kotikov, A.V.; Lipatov, A.V.; Parente, G.; Zotov, N.P. The Contribution of off-shell gluons to the structure functions $\mathrm{F}(2)^{* *} \mathrm{C}$ and $\mathrm{F}(\mathrm{L})^{* *} \mathrm{C}$ and the unintegrated gluon distributions. Eur. Phys. J. C 2002, $26,51$. [CrossRef]

203. Parisi, G.; Sourlas, N. A Simple Parametrization of the $Q^{2}$ Dependence of the Quark Distributions in QCD. Nucl. Phys. B 1979, 151, 421. [CrossRef]

204. Krivokhizhin, V.G.; Kotikov, A.V. Functions of the nucleon structure and determination of the strong coupling constant. Phys. Part. Nucl. 2009, 40, 1059. [CrossRef]

205. Kotikov, A.V.; Parente, G. Small x behavior of parton distributions with soft initial conditions. Nucl. Phys. B 1999, 549, 242. [CrossRef]

206. Illarionov, A.Y.; Kotikov, A.V.; Parente Bermudez, G. Small x behavior of parton distributions. A Study of higher twist effects. Phys. Part. Nucl. 2008, 39, 307. [CrossRef]

207. Kotikov, A.V. Deep inelastic scattering: $Q^{* * 2}$ dependence of structure functions. Phys. Part. Nucl. 2007, 38, 1; Erratum in 2007, 38, 828. [CrossRef]

(C) 2020 by the authors. Licensee MDPI, Basel, Switzerland. This article is an open access article distributed under the terms and conditions of the Creative Commons Attribution (CC BY) license (http:// creativecommons.org/licenses/by/4.0/). 